\title{
X-ray variability of Seyfert 1.8/1.9 galaxies
}

\author{
L. Hernández-García ${ }^{1,2}$, J. Masegosa ${ }^{1}$, O. González-Martín ${ }^{3}$, I. Márquez ${ }^{1}$, M. Guainazzi ${ }^{4}$, and F. Panessa ${ }^{2}$ \\ 1 Instituto de Astrofísica de Andalucía, CSIC, Glorieta de la Astronomía, s/n, 18008 Granada, Spain \\ e-mail: lorena.hernandez@iaps.inaf.it \\ 2 INAF-Istituto di Astrofisica e Planetologia Spaziali di Roma (IAPS-INAF), via del Fosso del Cavaliere 100, 00133 Roma, Italy \\ 3 Instituto de radioastronomía y Astrofísica (IRyA-UNAM), 3-72 (Xangari), 8701 Morelia, Mexico \\ ${ }^{4}$ European Space Research and Technology Centre (ESA/ESTEC), Kepleriaan 1, 2201 AZ Noordwijk, The Netherlands
}

Received 20 January 2017 / Accepted 13 March 2017

\begin{abstract}
Context. Seyfert 1.8/1.9 are sources showing weak broad $\mathrm{H}_{\alpha}$ components in their optical spectra. According to unification schemes, they are seen with an edge-on inclination, similar to type 2 Seyfert galaxies, but with slightly lower inclination angles.

Aims. We aim to test whether Seyfert 1.8/1.9 have similar properties at UV and X-ray wavelengths.

Methods. We used the 15 Seyfert 1.8/1.9 in the Véron Cetty and Véron catalog with public data available from the Chandra and/or $X M M-N e w t o n$ archives at different dates, with timescales between observations ranging from days to years. All the spectra of the same source were simultaneously fit with the same model and different parameters were left free to vary in order to select the variable parameter(s). Whenever possible, short-term variations from the analysis of the X-ray light curves and long-term UV variations from the optical monitor onboard XMM-Newton were studied. Our results are homogeneously compared with a previous work using the same methodology applied to a sample of Seyfert 2.

Results. X-ray variability is found in all 15 nuclei over the aforementioned ranges of timescales. The main variability pattern is related to intrinsic changes in the sources, which are observed in ten nuclei. Changes in the column density are also frequent, as they are observed in six nuclei, and variations at soft energies, possibly related to scattered nuclear emission, are detected in six sources. $\mathrm{X}$-ray intra-day variations are detected in six out of the eight studied sources. Variations at UV frequencies are detected in seven out of nine sources.

Conclusions. A comparison between the samples of Seyfert 1.8/1.9 and 2 shows that, even if the main variability pattern is due to intrinsic changes of the sources in the two families, these nuclei exhibit different variability properties in the UV and X-ray domains. In particular, variations in the broad X-ray band on short timescales (days to weeks), and variations in the soft X-rays and UV on long timescales (months to years) are detected in Seyfert 1.8/1.9 but not in Seyfert 2. Overall, we suggest that optically classified Seyfert 1.8/1.9 should be kept separated from Seyfert 2 galaxies in UV/X-ray studies of the obscured AGN population because their intrinsic properties might be different.
\end{abstract}

Key words. X-rays: galaxies - galaxies: active - ultraviolet: galaxies

\section{Introduction}

Active galactic nuclei (AGN) are thought to be powered by accretion of matter onto the supermassive black hole (SMBH) that resides in the center of the galaxies (Rees 1984). Historically, these nuclei have been classified as type 1 when broad Balmer permitted lines (full-width at half maximum $(F W H M) \sim$ $1000-20000 \mathrm{~km} \mathrm{~s}^{-1}$ ) are detected in their optical spectra, while they are classified as type 2 when detecting only narrow lines $\left(F W H M \sim 300-1000 \mathrm{~km} \mathrm{~s}^{-1}\right)$. Using the relative intensity of broad and narrow lines, the nuclei can also be classified as type $1.2,1.5,1.8$, or 1.9 AGN (intermediate Seyferts), the latter having the weaker broad component (e.g., Osterbrock 1977; Osterbrock \& Martel 1993). In particular, the optical spectra of Seyfert 1.8 are characterized by strong narrow emission lines combined with weak broad $\mathrm{H}_{\alpha}$ and $\mathrm{H}_{\beta}$ emission lines, whereas Seyfert 1.9 present the narrow lines but only a weak broad $\mathrm{H}_{\alpha}$ emission line (Osterbrock 1981).

The detection of broad components in polarized light of type 2 sources set the unified model of AGN (Lawrence et al. 1987; Antonucci 1993; Urry \& Padovani 1995; Moran et al. 2000). Under this scenario, the different properties observed in AGN can be explained by orientation effects, that is, they are the same kind of source observed at different angles. The cornerstone of this model is a dusty structure (often simplified as a torus) that surrounds the SMBH, which plays a fundamental role as it is responsible for obscuring the broad line region (BLR) where the broad lines are created. In support of this model, X-ray observations have shown that type 2 sources are more obscured than type 1s, whereas type 1.8 and 1.9 AGN are less absorbed than strictly type 2 s (Risaliti et al. 1999).

$\mathrm{X}$-rays are indeed a powerful tool for the comprehension of AGN as they are capable of reaching closer to the SMBH than other wavelengths. At these energies the absorbing column density, $N_{\mathrm{H}}$, is used to classify sources as unobscured (type 1) when $N_{\mathrm{H}}$ is below $\sim 10^{22} \mathrm{~cm}^{-2}$ and obscured (type 2) sources for larger values. For $N_{\mathrm{H}}$ values larger than $1.5 \times 10^{24} \mathrm{~cm}^{-2}$, the sources are classified as Compton-thick (Maiolino et al. 1998). Sometimes transitions from Compton-thick to Compton-thin (or vice versa) have been observed; these are known as changing-look sources according to the original nomenclature by Matt et al. (2003).

Variability is one of the properties characterizing AGN, a highly valuable tool for the comprehension of their physical structure (Peterson 1997; Netzer 2013). The first systematic studies of AGN showed that short-term X-ray variability (from 
hours to days) is common in type $1 \mathrm{~s}$, but not in type $2 \mathrm{~s}$, while long-term (from months to years) variations are common in both (e.g., Nandra et al. 1997; Turner et al. 1997; Vaughan et al. 2005). Currently, we believe that the X-ray variations might be related to intrinsic changes of the nuclear source (e.g., Uttley et al. 2005; Uttley 2007; Parker et al. 2015), or to absorbing clouds that intersect the line of sight to the observer (e.g., Risaliti et al. 2007). These changes can be studied by modeling the X-ray spectrum of AGN, whose continuum is dominated by a power-law component extending up to a cut-off at energies $\geq 100 \mathrm{keV}$ (e.g., Zdziarski et al. 1995; Guainazzi et al. 2005; Fabian et al. 2015). Changes in the power law might indicate a change in the accretion disk or the X-ray corona, while changes in the absorption may be related to clouds in our line of sight, more likely in the BLR, the torus, or the boundary between them (Risaliti et al. 2002, 2005a, 2011; Braito et al. 2013; Markowitz et al. 2014).

Because of their similar optical and X-ray spectra, it is usually assumed that optically classified Seyfert 1.2 and 1.5 behave more like type 1 sources, whereas types 1.8 and 1.9 behave as type 2 . Indeed, many studies aiming to analyze the properties of type 2 sources have included Seyfert 1.8/1.9 in their samples (e.g., Guainazzi et al. 2001; Risaliti 2002; Akylas \& Georgantopoulos 2009).

However, it is not clear whether the properties of Seyfert 1.8/1.9 are directly related to differences in the nuclear continuum or to an obscurer in our line of sight, since weaker broad lines may be produced by a lower ionizing continuum flux or by reddening from the BLR or the host galaxy (Osterbrock 1981; Goodrich 1995; Trippe et al. 2011). Through the analysis of variability, we are able to differentiate between changes in the accretion state and the configuration of the clouds. The main purpose of the present work is to homogeneously compare the variability properties of optically classified Seyfert 1.8/1.9 and Seyfert 2. The ultimate goal of our study is to understand the physical origin of the phenomenological differences between Seyfert 1.8/1.9 and Seyfert 2 in the optical, UV, and X-ray. We employ X-ray variability as gauge in this paper. This study is part of a systematic analysis of the variability properties of nearby AGN; by now we have analyzed the properties of a sample of optically classified low ionization nuclear emission line regions (LINERs, Hernández-García et al. 2013, 2014), and a sample of Seyfert 2 (Hernández-García et al. 2015). A comparison between the properties of LINERs and Seyfert 2 was carried out in Hernández-García et al. (2016).

The paper is organized as follows. The sample selection is presented in Sect. 2. The data reduction and the methodology are explained in Sects. 3 and 4. The results of the analysis are presented in Sect. 5, which are discussed in Sect. 6. Finally, the conclusions of this study are summarized in Sect. 7.

\section{Sample and data}

We used the 13th edition of the Véron-Cetty and Véron catalog (Véron-Cetty \& Véron 2010), which contains quasars and AGN. We selected nearby sources located at redshifts below $0.05^{1}$ that were classified as Seyfert type 1.8 and 1.9. In this way we selected 142 Seyfert 1.8 and 189 Seyfert 1.9.

\footnotetext{
1 The redshift of 0.05 corresponds to a distance of $d=214.3 \mathrm{Mpc}$ (using $H_{0}=70 \mathrm{~km} \mathrm{~s}^{-1}$ ). The limit on distance was chosen to be the same as in Hernández-García et al. (2015) for Seyfert 2.
}

We used the HEASARC database ${ }^{2}$ to search for public data in the Chandra and/or XMM-Newton archives of these sources. To study X-ray variability, we selected those sources with more than one observation with these satellites. This included 12 Seyfert 1.8 and another 12 Seyfert 1.9.

We further restricted our sample to sources whose spectra have a minimum of 400 number counts in the $0.5-10 \mathrm{keV}$ energy band (to use $\chi^{2}$-statistics) and to not be affected by a pileup fraction larger than $10 \%$. This leaves us with nine Seyfert 1.8 and seven Seyfert 1.9. We removed MARK 1018 from the sample because, although being classified as a Seyfert 1.9 by Osterbrock (1981) using optical data, Cohen et al. (1986) reported variations from Seyfert 1.9 to Seyfert 1 also using optical data, and remained as a Seyfert 1 at least up to 2007 (Trippe et al. 2010). Therefore, the final sample includes nine Seyfert 1.8 and six Seyfert 1.9. The sample properties are presented in Table 1.

We note that a caveat in the analysis could be related to the non simultaneity of the X-ray data with the optical spectroscopic data used for the optical classification of the sources. Unfortunately, the only case in our sample where the X-ray and optical data were obtained at close epochs is NGC 2617, where the X-ray data were taken in 2013, while it was reclassified as a Seyfert 1 using optical spectroscopy gathered in 2014, confirming that variability might be an important issue.

\section{Data reduction}

\subsection{Chandra data}

Chandra observations were obtained from the ACIS instrument (Garmire et al. 2003). Data reduction and analysis were carried out in a systematic, uniform way using CXC Chandra Interactive Analysis of Observations $\left(\mathrm{CIAO}^{3}\right)$, version 4.6. Level 2 event data were extracted by using the task ACIS-PROCESS-EVENTS. Background flares were cleaned using the task LC_CLEAN.SL ${ }^{4}$, which calculates a mean rate from which it deduces a minimum and maximum valid count rate and creates a good time intervals file.

Nuclear spectra were extracted from a circular region centered on the positions given by $\mathrm{NED}^{5}$. We chose circular radii, aiming to include all possible photons, while excluding other sources or background effects. The radii are in the range between 2-4" (see Table A.1). The background was extracted from circular regions in the same chip that are free of sources and close to the object.

For the source and background spectral extractions, the DMEXTRACT task was used. The response matrix file (RMF) and ancillary reference file (ARF) were generated for each source region using the MKACISRMF and MKWARF tasks, respectively. Finally, the spectra were binned to have a minimum of 20 counts per spectral bin using the GRPPHA task (included in FTOOLS), to be able to use the $\chi^{2}$-statistics, as customary in X-ray spectroscopy.

\subsection{XMM-Newton data}

$X M M$-Newton observations were obtained with the EPIC pn camera (Strüder et al. 2001). The data were reduced in a systematic, uniform way using the Science Analysis Software $\left(\mathrm{SAS}^{6}\right)$,

\footnotetext{
2 http://heasarc.gsfc.nasa.gov/

http://cxc.harvard.edu/ciao4.4/

http://cxc.harvard.edu/ciao/ahelp/lc_clean.html

http://ned.ipac.caltech.edu/

6 http://xmm.esa.int/sas/
} 
Table 1. General properties of the sample galaxies.

\begin{tabular}{|c|c|c|c|c|c|c|c|c|}
\hline Name & $\begin{array}{c}\text { RA } \\
(\mathrm{J} 2000) \\
(2)\end{array}$ & $\begin{array}{c}\text { Dec } \\
(\mathrm{J} 2000) \\
(3)\end{array}$ & $\begin{array}{l}\text { Dist. }^{1} \\
(\mathrm{Mpc}) \\
(4)\end{array}$ & $\begin{array}{c}N_{\mathrm{Gal}} \\
\left(10^{20} \mathrm{~cm}^{-2}\right) \\
(5)\end{array}$ & (6) & $\begin{array}{l}\text { Morph. } \\
\text { type } \\
(7)\end{array}$ & $\begin{array}{l}\text { Seyfert } \\
\text { type } \\
(8)\end{array}$ & $\begin{array}{c}\log M_{\mathrm{BH}} \\
M_{\odot} \\
(9)\end{array}$ \\
\hline ESO 540-G01 & 003413.8 & -212620 & 110.5 & 1.62 & 13.7 & $\mathrm{SBc}$ & 1.8 & - \\
\hline ESO 195-IG21 & 010036.5 & -475203 & 201.8 & 1.65 & 16.7 & - & 1.8 & - \\
\hline ESO 113-G10 & 010517.0 & -582613 & 104.1 & 2.95 & 14.6 & $\mathrm{SBa}$ & 1.8 & 6.85 \\
\hline NGC 526A & 012354.4 & -350356 & 77.8 & 2.19 & 14.6 & So & 1.9 & 7.90 \\
\hline MARK 609 & 032525.4 & -060839 & 141.1 & 4.42 & 14.1 & So & 1.8 & - \\
\hline NGC 1365 & 033336.4 & -360824 & 18.0 & 1.35 & 13.0 & $\mathrm{Sb}$ & 1.8 & 7.54 \\
\hline NGC 2617 & 083538.8 & -040519 & 56.1 & 3.65 & 14.0 & $\mathrm{SBc}$ & 1.8 & 7.60 \\
\hline MARK 1218 & 083811.1 & 245345 & 116.6 & 3.54 & 14.1 & $\mathrm{Sb}$ & 1.8 & - \\
\hline NGC 2992 & 094542.0 & -141935 & 30.5 & 4.99 & 13.8 & $\mathrm{Sa}$ & 1.9 & 7.73 \\
\hline POX 52 & 120256.8 & -205603 & 87.3 & 4.03 & 17.2 & - & 1.8 & 5.14 \\
\hline NGC 4138 & 120929.9 & 434106 & 16.0 & 1.36 & 12.2 & S0-a & 1.9 & 7.30 \\
\hline NGC 4395 & 122548.9 & 333248 & 4.5 & 1.35 & 10.3 & $\mathrm{Sm}$ & 1.8 & 4.82 \\
\hline NGC 4565 & 123620.6 & 255911 & 12.1 & 1.30 & 12.4 & $\mathrm{Sb}$ & 1.9 & 6.30 \\
\hline MARK 883 & 162952.8 & 242639 & 155.7 & 3.97 & 14.4 & I & 1.9 & 7.28 \\
\hline IRAS 20051-1117 & 200751.4 & -110835 & 128.9 & 6.57 & 14.0 & - & 1.9 & 7.11 \\
\hline
\end{tabular}

Notes. (Column 1) Name, (Col. 2) right ascension, (Col. 3) declination, (Col. 4) distance, (Col. 5) galactic absorption, (Col. 6) aparent magnitude in the Johnson filter $V$ from Véron-Cetty \& Véron (2010), (Col. 7) galaxy morphological type from Hyperleda, (Col. 8) AGN type as in Véron-Cetty \& Véron (2010), and (Col. 9) black-hole mass on logarithmical scale, determined using the correlation between stellar velocity dispersion (from HyperLeda) and black-hole mass (Tremaine et al. 2002), or obtained from the literature otherwise (ESO 113-G10 from Cackett et al. 2013, NGC 526A from Vasudevan \& Fabian 2009, NGC 2617 from Shappee et al. 2014, MARK 883 from Benítez et al. 2013, and IRAS 200511117 from Wang \& Zhang 2007). ${ }^{(1)}$ All distances are taken from the NED and correspond to the average redshift-independent distance estimates.

version 14.0.0. First, good time intervals were selected using a method that maximizes the signal-to-noise $(\mathrm{S} / \mathrm{N})$ of the net source spectrum by applying a different constant count rate threshold on the single events, $E>10 \mathrm{keV}$ field-of-view background light curve. We extracted the spectra of the nuclei from circles of 20-35" radius centered on the positions given by NED, while the background spectra were extracted from circular regions using an algorithm that automatically selects the best area - and the closest to the source - that is free of sources. This selection was manually checked to ensure the best selection for the backgrounds.

Source and background spectra were extracted with the EVSELECT task. The response matrix files (RMF) and the ancillary response files (ARF) were generated using the RMFGEN and ARFGEN tasks, respectively. To be able to use the $\chi^{2}$ statistics, the spectra were binned to obtain at least 20 counts per spectral bin using the GRPPHA task.

\subsection{Light curves}

Light curves in three energy bands $(0.5-2.0 \mathrm{keV}, 2.0-10.0 \mathrm{keV}$, and $0.5-10 \mathrm{keV}$ ) for the source and background regions as defined above were extracted using the DMEXTRACT task (for XMM-Newton) and EVSELECT task (for Chandra) with a 1000s bin. To be able to compare the variability amplitudes in different light curves of the same object, only those observations with a net exposure time longer than $30 \mathrm{ks}$ were taken into account. For observations longer than $40 \mathrm{ks}$, the light curves were divided into segments of $40 \mathrm{ks}$, so in some cases more than one segment of the same light curve can be extracted. Our light curves are occasionally affected by high particle background events ("flares"), whose flux dominates the observed count rates. We decided to remove these intervals from the source background-subtracted light curves due to their poor $\mathrm{S} / \mathrm{N}$ that could affect the estimate of the normalized effect variance (cf. Sect. 4.4). High particle background flux intervals were identified using the same algorithm described in Sect. 3.2. As the fraction of high-particle background intervals is small, our procedure does not significantly affect the results discussed in this paper (Vaughan et al. 2003). We notice that after excluding these events, the exposure time of the light curve could be shorter, thus we recall that only observations with a net exposure time longer than $30 \mathrm{ks}$ were used for the analysis. The light curves are shown in Appendix D. We recall that the values of the continuum (median value of the count rate) and dashed ( $1 \sigma$ standard deviation) lines are used only for visual inspection of the data and not as estimators of the variability (as in Hernández-García et al. 2014).

\section{Method}

The method used in this work is presented in Hernández-García et al. (2013). Here we review the most important aspects but we refer the reader to this paper for further details of the analysis.

\subsection{Individual spectral analysis}

The first step is to select a model to fit all the data of the same source simultaneously. For that purpose, we used five different models that were fitted to each spectrum individually. We note that more complex models were also tested but they were not required by the data. The models are as follows:

- PL: a single power law representing the continuum of a nonstellar source. The empirical model is $e^{N_{\mathrm{Gal}} \sigma(E)} \cdot e^{N_{\mathrm{H}} \sigma(E(1+z))}\left[N_{\mathrm{H}}\right] \cdot$ Norm $e^{-\Gamma}[\Gamma$, Norm $]$.

- ME: the emission is dominated by hot diffuse gas, that is, a thermal plasma. A MEKAL (in XSPEC) model is used to fit the spectrum. The model is $e^{N_{\mathrm{Gal}} \sigma(E)} \cdot e^{N_{\mathrm{H}} \sigma(E(1+z))}\left[N_{\mathrm{H}}\right] \cdot \operatorname{MEKAL}[k T$, Norm $]$. 
- 2PL: in this model the primary continuum is an absorbed power law representing the non stellar source, while the soft energies are due to a scattering component that is represented by another power law. Mathematically the model is explained as

$e^{N_{\mathrm{Gal}} \sigma(E)}\left(e^{N_{\mathrm{H} 1} \sigma(E(1+z))}\left[N_{\mathrm{H} 1}\right] \quad \cdot \quad \operatorname{Norm}_{1} e^{-\Gamma}\left[\Gamma\right.\right.$, Norm $\left._{1}\right]+$ $e^{N_{\mathrm{H} 2} \sigma(E(1+z))}\left[N_{\mathrm{H} 2}\right] \cdot \operatorname{Norm}_{2} e^{-\Gamma}\left[\Gamma\right.$, Norm $\left.\left._{2}\right]\right)$.

- MEPL: the primary continuum is represented by an absorbed power law, but at soft energies a thermal plasma dominates the spectrum. Empirically it can be described as $e^{N_{\mathrm{Gal}} \sigma(E)}\left(e^{N_{\mathrm{H} 1} \sigma(E(1+z))}\left[N_{\mathrm{H} 1}\right] \cdot \operatorname{MEKAL}\left[k T, \mathrm{Norm}_{1}\right]+\right.$ $\left.e^{N_{\mathrm{H} 2} \sigma(E(1+z))}\left[N_{\mathrm{H} 2}\right] \cdot \operatorname{Norm}_{2} e^{-\Gamma}\left[\Gamma, \mathrm{Norm}_{2}\right]\right)$.

- ME2PL: same model as MEPL, but an additional power law is required to explain the scattered component at soft energies, so mathematically it is

$e^{N_{\mathrm{Gal}} \sigma(E)}\left(e^{N_{\mathrm{H} 1} \sigma(E(1+z))}\left[N_{\mathrm{H} 1}\right] \quad \cdot \quad \operatorname{Norm}_{1} e^{-\Gamma}\left[\Gamma\right.\right.$, Norm $\left._{1}\right]+$ $\left.\operatorname{MEKAL}[k T]+e^{N_{\mathrm{H} 2} \sigma(E(1+z))}\left[N_{\mathrm{H} 2}\right] \cdot \operatorname{Norm}_{2} e^{-\Gamma}\left[\Gamma, \mathrm{Norm}_{2}\right]\right)$.

In the equations above, $\sigma(E)$ is the photo-electric cross-section, $z$ is the redshift, and Norm $_{i}$ are the normalizations of the power law and/or the thermal component. For each model, the parameters that vary are written in brackets. The Galactic absoption, $N_{\mathrm{Gal}}$, is included in each model and fixed to the predicted value (Col. 5 in Table 1) using the tool NH within FTOOLS (Dickey \& Lockman 1990; Kalberla et al. 2005). Even if not included in the mathematical expressions above, all the models include three narrow Gaussian lines to take the iron lines at $6.4 \mathrm{keV}(\mathrm{FeK} \alpha), 6.7 \mathrm{keV}$ (FeXXV), and $6.95 \mathrm{keV}$ (FeXXVI) into account.

The $\chi^{2} /$ d.o.f. and $F$-test were used to select the simplest model that represents the data best. We considered an improvement of the spectral fit significant when the $F$-test results in a value lower than $10^{-5}$.

\subsection{Simultaneous spectral analysis}

We determined the best-fit model for each individual observation using the procedure described in Sect. 4.1. As a baseline model we used the one corresponding to the individual observation with the largest count number, and we checked that it matches the best fit model of the remaining spectra of the same source ${ }^{7}$. This model was applied to all the observations of the same source simultaneously with its parameters linked amongst them - we note that the values of the parameters are able to change from the initial values given in the baseline model. If this fit (SMF0) resulted in a good fit (see below), we considered the source as non-variable.

When SMF0 did not give a good result, the next step was to let different parameters in the model vary one-by-one (SMF1). These parameters are the column densities at soft $\left(N_{\mathrm{H} 1}\right)$ and hard $\left(N_{\mathrm{H} 2}\right)$ energies, the temperature $(k T)$, the spectral index $(\Gamma)$, and the normalizations at soft $\left(\mathrm{Norm}_{1}\right)$ and hard $\left(\mathrm{Norm}_{2}\right)$ energies.

When SMF1 failed to be a good fit, we also tested to vary two parameters at the same time (SMF2), and also three parameters (SMF3) were needed in one case.

Each "next step" (e.g., SMF1 versus SMF0) was always tested in order to confirm an improvement of the spectral fit. A $\chi_{r}^{2}$ in the range between $0.9-1.5-$ and as close as possible to the unity and an $F$-test value lower than $10^{-5}$ were the criteria

\footnotetext{
7 We note that for NGC 4138 we used the PL model because the Chandra spectrum did not have counts below $2 \mathrm{KeV}$, therefore the analysis was performed in the $2-10 \mathrm{keV}$ band.
}

to accept a new step. If different models at a given step yielded a significant improvement with respect to the previous step, we chose the model corresponding to the lowest $\chi_{r}^{2}$.

Whenever possible, this analysis was applied to observations of the same satellite. However, in some cases there was only one observation per instrument available. In order to compare the data extracted from different apertures, we fit the extranuclear emission in the annular region in the Chandra image between the Chandra aperture around the nucleus and the XMM-Newton aperture (see Table A.1) using the same procedure described in Sect. 4.1. This allowed us to define the best-fit model of the Chandra extranuclear emission. This model was included in the spectral analysis of the XMM-Newton data, when comparing with Chandra data. This procedure was applied whenever $X M M-N e w t o n$ and Chandra data were available.

\subsection{Flux variability}

The luminosities in the soft and hard X-ray energy bands were computed using XSPEC for both the fits of the individual observations, as well as for the simultaneous fit of all the observations together. The distances were taken from NED, corresponding to the average redshift-independent distance estimate for each object, when available, or to the redshift-estimated distance otherwise; distances are listed in Table 1.

When data from the optical monitor (OM) onboard XMMNewton were available, UV luminosities (simultaneously to $\mathrm{X}$-ray data) were estimated in the available filters. We recall that $U V W 2$ is centered at $1894 \AA(1805-2454) \AA, U V M 2$ at $2205 \AA$ (1970-2675) $\AA$, and $U V W 1$ at $2675 \AA(2410-3565) \AA$. We used the OM observation FITS source lists (OBSMLI) ${ }^{8}$ to obtain the photometry. When OM data were not available, we searched for UV information in the literature. We note that in this case, the $\mathrm{X}$-ray and UV data might not be simultaneous (see Appendix B).

We assumed an object to be variable when the square root of the squared errors was at least three times smaller than the dynamical range covered by the luminosities (see Hernández-García et al. 2014, for details).

\subsection{Short-term variability}

Initially, we assumed a constant count rate for segments of 30$40 \mathrm{ks}$ of the observation in each energy band and calculated the $\chi^{2} /$ degrees of freedom (d.o.f.). We considered the source as a variable candidate if the count rate differed from the average by more than $3 \sigma$ (or $99.7 \%$ probability).

Secondly, we calculated the normalized excess variance, $\sigma_{\text {NXS }}^{2}$, for each light curve segment with 30-40 ks following prescriptions in Vaughan et al. (2003, see also González-Martín et al. 2011; Hernández-García et al. 2014). We recall that $\sigma_{\mathrm{NXS}}^{2}$ is related to the area below the power spectral density (PSD) shape.

When $\sigma_{\text {NXS }}^{2}$ was negative or compatible with zero within the errors, we estimated the $90 \%$ upper limits using Table 1 in Vaughan et al. (2003). We assumed a PSD slope of -1 , the upper limit from Vaughan et al. (2003), and we added the value of 1.282err $\left(\sigma_{\mathrm{NXS}}^{2}\right)$ to the limit to account for Poisson noise. For a number of segments, $\mathrm{N}$, obtained from an individual light curve, an upper limit for the normalized excess variance was calculated. When $N$ segments were obtained for the same light curve and at least one was consistent with being variable, we calculated the

\footnotetext{
8 ftp://xmm2.esac.esa.int/pub/odf/data/docs/ XMM-SOC-GEN-ICD-0024.pdf
} 
Table 2. Results of the variability analysis.

\begin{tabular}{|c|c|c|c|c|c|c|c|c|c|}
\hline Name & $\log \left(L_{\mathrm{soft}}\right)$ & $\log \left(L_{\mathrm{hard}}\right)$ & $\log \left(R_{\mathrm{Edd}}\right)$ & Long- & term var & ability & $\Delta T_{\max }$ & Short & UV \\
\hline (1) & $\begin{array}{c}(0.5-2 \mathrm{keV}) \\
(2)\end{array}$ & $\begin{array}{c}(2-10 \mathrm{keV}) \\
(3)\end{array}$ & (4) & $\begin{array}{l}\text { SMF0 } \\
(5)\end{array}$ & $\begin{array}{l}\text { SMF1 } \\
(6)\end{array}$ & $\begin{array}{c}\text { SMF } 2 / 3 \\
\text { (7) }\end{array}$ & $\begin{array}{c}\text { (years) } \\
(8)\end{array}$ & $\begin{array}{l}\text { term } \\
(9)\end{array}$ & $\begin{array}{l}\text { Variab. } \\
(10)\end{array}$ \\
\hline ESO 540-G01 (X, C) & $\begin{array}{c}41.53 \pm 0.13 \\
40 \%\end{array}$ & $\begin{array}{c}41.72 \pm 0.13 \\
38 \%\end{array}$ & - & MEPL & $\begin{array}{c}\text { Norm }_{2} \\
74 \%\end{array}$ & & 1 & - & - \\
\hline ESO 195-IG21 (X, C) & $\begin{array}{c}42.54 \pm 0.37 \\
98 \%\end{array}$ & $\begin{array}{c}43.03 \pm 0.37 \\
97 \%\end{array}$ & - & MEPL & $\begin{array}{c}\mathrm{Norm}_{2} \\
91 \%\end{array}$ & $\begin{array}{l}\text { Norm }_{1} \\
98 \%\end{array}$ & 4 & - & - \\
\hline ESO $113-G 10(X)$ & $\begin{array}{c}43.07 \pm 0.05 \\
17 \%\end{array}$ & $\begin{array}{c}42.70 \pm 0.05 \\
17 \%\end{array}$ & -0.74 & ME2PL & $\begin{array}{l}\text { Norm }_{1} \\
40 \%\end{array}$ & & 4 & TSH & W2 \\
\hline NGC 526A (X) & $\begin{array}{c}42.91 \pm 0.10 \\
48 \%\end{array}$ & $\begin{array}{c}43.32 \pm 0.09 \\
46 \%\end{array}$ & -1.16 & $2 \mathrm{PL}$ & $\begin{array}{c}\mathrm{Norm}_{2} \\
48 \%\end{array}$ & - & 11 & TSH & W1 \\
\hline MARK 609 (X) & $\begin{array}{c}42.55 \pm 0.04 \\
13 \%\end{array}$ & $\begin{array}{c}42.69 \pm 0.04 \\
13 \%\end{array}$ & - & $2 \mathrm{PL}$ & $\begin{array}{l}\text { Norm }_{1} \\
22 \%\end{array}$ & & 5 & - & - \\
\hline NGC $1365(X)^{C L}$ & $\begin{array}{c}41.15 \pm 0.35 \\
81 \%\end{array}$ & $\begin{array}{c}42.18 \pm 0.42 \\
24 \%\end{array}$ & -1.95 & ME2PL & $\begin{array}{l}N_{\mathrm{H} 2} \\
68 \%\end{array}$ & $\begin{array}{l}N 2 / N 1^{*} \\
33 / 35 \%\end{array}$ & 10 & TSH & $\mathrm{W} 1, \mathrm{M} 2$ \\
\hline$(\mathrm{X}, \mathrm{C})$ & $\begin{array}{c}41.80 \pm 0.38 \\
99 \%\end{array}$ & $\begin{array}{c}41.73 \pm 0.27 \\
77 \%\end{array}$ & & ME2PL & $\begin{array}{l}N_{\mathrm{H} 2} \\
37 \%\end{array}$ & $\begin{array}{c}\text { Norm }_{2} \\
30 \%\end{array}$ & 2 & - & - \\
\hline NGC $2617(\mathrm{X})$ & $\begin{array}{c}43.24 \pm 0.15 \\
46 \%\end{array}$ & $\begin{array}{c}43.25 \pm 0.15 \\
45 \%\end{array}$ & -0.94 & $2 \mathrm{PL}$ & $\begin{array}{l}\text { Norm }_{1} \\
59 \%\end{array}$ & $\begin{array}{l}N_{\mathrm{H} 2} \\
30 \%\end{array}$ & 0.1 & $\mathrm{TS}$ & W1 \\
\hline MARK $1218(X)$ & $\begin{array}{c}41.87 \pm 0.22 \\
64 \%\end{array}$ & $\begin{array}{c}42.56 \pm 0.21 \\
64 \%\end{array}$ & - & PL & $\begin{array}{l}\text { Norm } \\
63 \%\end{array}$ & & 0.08 & - & No \\
\hline NGC $2992(X)^{C L}$ & $\begin{array}{c}41.57 \pm 0.23 \\
19 \%\end{array}$ & $\begin{array}{c}42.03 \pm 0.22 \\
19 \%\end{array}$ & -2.30 & $2 \mathrm{PL}$ & $\begin{array}{c}N_{\mathrm{H} 2} \\
5 \%\end{array}$ & $\begin{array}{c}\mathrm{Norm}_{2} \\
21 \%\end{array}$ & 3 & $\mathrm{TH}$ & M2 \\
\hline POX $52(X, C)$ & $\begin{array}{c}41.89 \pm 0.01 \\
2 \%\end{array}$ & $\begin{array}{c}41.75 \pm 0.02 \\
7 \%\end{array}$ & 0.18 & ME2PL & $\begin{array}{l}N_{\mathrm{H} 2} \\
44 \%\end{array}$ & $\begin{array}{c}N_{\mathrm{H} 1} \\
100 \%\end{array}$ & 1 & No & - \\
\hline NGC $4138(X, C)$ & - & $\begin{array}{c}41.53 \pm 0.07 \\
21 \%\end{array}$ & -2.42 & $\mathrm{PL}^{* *}$ & $\begin{array}{l}\text { Norm } \\
98 \%\end{array}$ & & 2 & - & - \\
\hline NGC 4395 (C) & $\begin{array}{c}39.50 \\
0 \%\end{array}$ & $\begin{array}{c}39.94 \\
0 \%\end{array}$ & -1.15 & ME2PL & $\begin{array}{l}N_{\mathrm{H} 2} \\
31 \%\end{array}$ & & 0.003 & - & W1 \\
\hline$(\mathrm{X})$ & $\begin{array}{c}39.69 \pm 0.06 \\
15 \%\end{array}$ & $\begin{array}{c}40.28 \pm 0.40 \\
13 \%\end{array}$ & & ME2PL & $\begin{array}{l}N_{\mathrm{H} 2} \\
20 \%\end{array}$ & $\begin{array}{c}\mathrm{Norm}_{2} \\
88 \%\end{array}$ & 12 & TSH & - \\
\hline$(\mathrm{X}, \mathrm{C})$ & $\begin{array}{c}39.78 \pm 0.21 \\
61 \%\end{array}$ & $\begin{array}{c}40.21 \pm 0.22 \\
65 \%\end{array}$ & & ME2PL & $\begin{array}{c}\mathrm{Norm}_{2} \\
93 \%\end{array}$ & & 2 & - & - \\
\hline NGC $4565(X, C)$ & $\begin{array}{c}39.51 \pm 0.03 \\
11 \%\end{array}$ & $\begin{array}{c}39.65 \pm 0.07 \\
21 \%\end{array}$ & -4.63 & PL & $\begin{array}{c}N_{\mathrm{H}} \\
48 \%\end{array}$ & & 2 & No & - \\
\hline MARK $883(\mathrm{X})$ & $\begin{array}{c}42.42 \pm 0.09 \\
28 \%\end{array}$ & $\begin{array}{c}42.71 \pm 0.08 \\
28 \%\end{array}$ & -1.15 & PL & $\begin{array}{l}\text { Norm } \\
28 \%\end{array}$ & & 4 & - & $\mathrm{W} 1, \mathrm{~W} 2$ \\
\hline IRAS 20051-1117 (X) & $\begin{array}{c}42.39 \pm 0.09 \\
29 \%\end{array}$ & $\begin{array}{c}42.53 \pm 0.09 \\
29 \%\end{array}$ & -1.16 & PL & $\begin{array}{l}\text { Norm } \\
29 \%\end{array}$ & & 0.5 & - & No \\
\hline
\end{tabular}

Notes. (Column 1) name, and the instrument (C: Chandra and/or X: XMM-Newton) in parenthesis ( ${ }^{\mathrm{CL}}$ refer to changing-look candidates); (Cols. 2 and 3) logarithm of the soft $(0.5-2 \mathrm{keV})$ and hard $(2-10 \mathrm{keV}) \mathrm{X}$-ray luminosities, where the mean was calculated for variable objects, and percentages in flux variations; (Col. 4) Eddington ratio, $L_{\mathrm{bol}} / L_{\mathrm{Edd}}$, calculated from Eracleous et al. (2010) using $L_{\mathrm{bol}}=33 L_{2-10 \mathrm{keV}}$; (Col. 5) best fit for SMF0; (Col. 6) parameter varying in SMF1, with the percentage of variation; (Col. 7) parameter varying in SMF2 and SMF3 (for NGC 1365, (*) $\mathrm{N} 1=\mathrm{Norm}_{1}$, and $\mathrm{N} 2=\mathrm{Norm}_{2}$ ), with the percentage of variation; (Col. 8) the sampling timescale, corresponding to the difference between the first and the last observation. The percentages correspond to this $\Delta T_{\max }$; (Col. 9) short-term variations in the total (T), soft $(\mathrm{S})$, and/or hard $(\mathrm{H})$ energy bands; and (Col. 10) filters where variations are detected at UV frequencies with the OM. A "_" means that data were not available, while "No" means that variations were not detected. ${ }^{(* *)}$ We note that the XMM-Newton data of NGC 4138 is best fitted by the ME2PL model, but Chandra data does not have counts below $2 \mathrm{keV}$, thus the PL model is used for the simultaneous fit.

normalized weighted mean and its error as the weighted variance. We considered short-term variations for $\sigma_{\mathrm{NXS}}^{2}$ detections above $3 \sigma$ of the confidence level.

\subsection{Compton thickness}

We tested the possibility of some sources being so heavily absorbed that their spectra can be completely reflected below
$10 \mathrm{keV}$, i.e., Compton-thick sources. Since the Compton-thick column densities cannot be directly measured at the energies analyzed here, the following indirect indicators (using X-ray and $[\mathrm{O}$ III] data) are taken to classify these sources: $\Gamma<$ $1, E W(\mathrm{FeK} \alpha)>500 \mathrm{eV}$, and $F(2-10 \mathrm{keV}) / F_{[\text {OIII] }}<1$ (Ghisellini et al. 1994; Bassani et al. 1999; Panessa \& Bassani 2002). Where $\Gamma$ and $\mathrm{EW}(\mathrm{FeK} \alpha)$ were obtained from individual spectral fits in the 3-10 keV energy band using the PL model, the extinction-corrected [O III] fluxes were obtained from the 
Table 3. Median values and $25 \%$ and $75 \%$ percentiles of the spectral parameters of Seyfert 1.8/1.9 presented in this work (Col. 2) and the Seyfert 2 sample (Col. 3) presented in Hernández-García et al. (2015).

\begin{tabular}{lcc}
\hline \hline & $\begin{array}{c}\text { Seyfert 1.8/1.9 } \\
(1)\end{array}$ & $\begin{array}{c}\text { Seyfert 2 } \\
(2)\end{array}$ \\
\hline $\log \left(L(0.5-2 \mathrm{keV})\left[\mathrm{erg} \mathrm{s}^{-1}\right]\right)$ & $41.9_{41.2}^{42.6}$ & $42.1_{41.3}^{42.6}$ \\
$\log \left(L(2-10 \mathrm{keV})\left[\mathrm{erg} \mathrm{s}^{-1}\right]\right)$ & $42.5_{41.7}^{42.7}$ & $42.7_{42.5}^{42.8}$ \\
$N_{\mathrm{H} 2}\left(\times 10^{22}\left[\mathrm{~cm}^{-2}\right]\right)$ & $3.00_{0.06}^{8.34}$ & $22.2_{9.8}^{38.4}$ \\
$\Gamma$ & $1.7_{1.4}^{1.9}$ & $1.7_{1.5}^{2.0}$ \\
$k T[\mathrm{keV}]$ & $0.19_{0.09}^{0.38}$ & $0.71_{0.67}^{0.81} / 0.15_{0.12}^{0.18}$ \\
$\mathrm{~S} / \mathrm{N}(0.5-2 \mathrm{KeV})$ & $5.3_{4.9}^{7.7}$ & $5.2_{3.8}^{6.4}$ \\
$\mathrm{~S} / \mathrm{N}(2-10 \mathrm{KeV})$ & $5.4_{4.7}^{6.6}$ & $3.8_{2.3}^{5.2}$ \\
\hline
\end{tabular}

Notes. (Column 1) spectral parameter, (Col. 2) average values for Seyfert 1.8/1.9, and (Col. 3) average values of Seyfert 2 from Hernández-García et al. (2015). The two temperatures represent the two thermal components in the model.

literature (and corrected when needed following Bassani et al. 1999), and the hard X-ray luminosities, $L(2-10 \mathrm{keV})$, from the individual fits were used (see Table A.3) for the calculation.

We considered that a source is a Compton-thick candidate when at least two of the three criteria above were met. Otherwise, the source is considered to be a Compton-thin candidate. When different observations of the same source result in different classifications, the object was considered to be a changing-look candidate.

\section{Results}

In this section we present the results of the spectral characteristics and variability patterns of the Seyfert 1.8/1.9 in the sample. For results on individual sources we refer the reader to Appendix B, as well as for notes and comparison with previous studies.

\subsection{Spectral characteristics}

We used five different models to fit each spectrum individually. The best model for each source resulted to be the same in all the individual observations from the same satellite. When comparing data from different instruments, different best fit models were selected for two sources (NGC 1365, and NGC 4138), most probably because of the low count-rate in the Chandra data, which required the simplest model. It is worth noticing that the XMM-Newton spectrum of NGC 4138 is best fitted with the ME2PL model, but the lack of counts in the Chandra spectrum below $2 \mathrm{keV}$ forced us to perform the analysis only above $2 \mathrm{KeV}$ and thus using the PL model (see Appendix B.11). The ME model was not the best-fit for any of the spectra.

The median (25\% and $75 \%$ percentiles) values of the spectral parameters are presented in Table 3. Absorption at soft energies is usually compatible with the Galactic one (see Table A.2). Absorption at high energies is common in these sources, being obscured in the range of $10^{21}-10^{23} \mathrm{~cm}^{-2}$, with median of $N_{\mathrm{H} 2}=3.00[0.06-8.34] \times 10^{22} \mathrm{~cm}^{-2}$. The median value of the spectral indices is $\Gamma=1.7[1.4-1.9]$, completely compatible with other AGN (see e.g., Brightman \& Nandra 2011a). The thermal component has a median of $k T=0.19[0.09-0.62] \mathrm{keV}$.
The X-ray luminosity medians in our sample are $\log L(0.5-$ $2.0 \mathrm{keV})=41.9[41.2-42.6]$ and $\log L(2-10 \mathrm{keV})=42.5[41.7-$ 42.7].

\subsection{Compton-thickness}

We recall that a source was classified as a Compton-thick candidate within an observation when at least two out of the three criteria explained in Sect. 4.5 were met. None of the sources are classified as Compton-thick. Two of the sources are classified as changing-look candidates (NGC 1365, and NGC 2992), as already reported in the literature (Gilli et al. 2000; Risaliti et al. 2009). Another two sources have been classified as changinglook candidates in the literature (MARK 609, Trippe et al. 2010, and NGC 2617; Shappee et al. 2014), but the present work does not detect these changes. It is worth noticing that we did not find the flux of the [OIII] in the literature for four sources (ESO 540-G01, ESO 195-IG21, ESO 113-G10, and NGC 2617), but the two other criteria were compatible with them being Compton-thin.

\subsection{Long-term $X$-ray spectral variability}

From the 15 nuclei in our sample, we compare spectra obtained from the same instrument in 10 cases, all of them observed by XMM-Newton, and in one case (NGC 4395) Chandra data are also available. In the remaining five sources only one observation per instrument was available.

Chandra and XMM-Newton data are available for the same source in eight cases (note that this analysis is independent of the one mentioned above, see Table A.1), thus the simultaneous analysis was carried out by using the methodology explained in Sect. 4.2.

Long-term X-ray spectral variability is detected in all the 15 nuclei. Variations are detected in four parameters Norm $_{1}$, Norm $_{2}, N_{\mathrm{H} 1}$, and $N_{\mathrm{H} 2}$ ). In nine objects the observed variability can be explained by varying only one parameter; in five nuclei varying two parameters is required (ESO 195IG21, NGC 2617, NGC 2992, POX 52, and NGC 4395), and in NGC 1365 varying three parameters is required. The most frequent variations are found in $\mathrm{Norm}_{2}$, which are observed in ten nuclei (ESO 540-G01, ESO 195-IG21, NGC 526A, NGC 1365, MARK 1218, NGC 2992, NGC 4138, NGC 4395, MARK 883, and IRAS 20051-1117). Changes in $N_{\mathrm{H} 2}$ are also frequent, as they are observed in six nuclei (NGC 1365, NGC 2617, NGC 2992, POX 52, NGC 4395, and NGC 4565). Variations at soft energies are detected in six sources (ESO 195IG21, ESO 113-G01, MARK 609, NGC 1365, NGC 2617, and POX 52). Among them, only in two objects (ESO 195-IG21 and POX 52) these variations are reported for a simultaneous fit using Chandra and XMM-Newton together, thus these variations cannot be ascribed to the comparison of data obtained from different instruments.

\subsection{Short-term $X$-ray variability}

Short-term X-ray variations are analyzed in eight nuclei (Table A.4). We recall that only light curves longer than $30 \mathrm{ks}$ were analyzed (see Sect. 4.4). Two sources do not show variations (POX 52 and NGC 4565) according to the $\chi^{2} /$ d.o.f. and $\sigma_{\text {NXS }}^{2}$, whereas the remaining six are variable in at least one energy band. Four sources show variations in the total, soft, and hard energy bands (ESO 113-G10, NGC 526A, NGC 1365, 
L. Hernández-García et al.: X-ray variability in Seyfert 1.8/1.9 galaxies
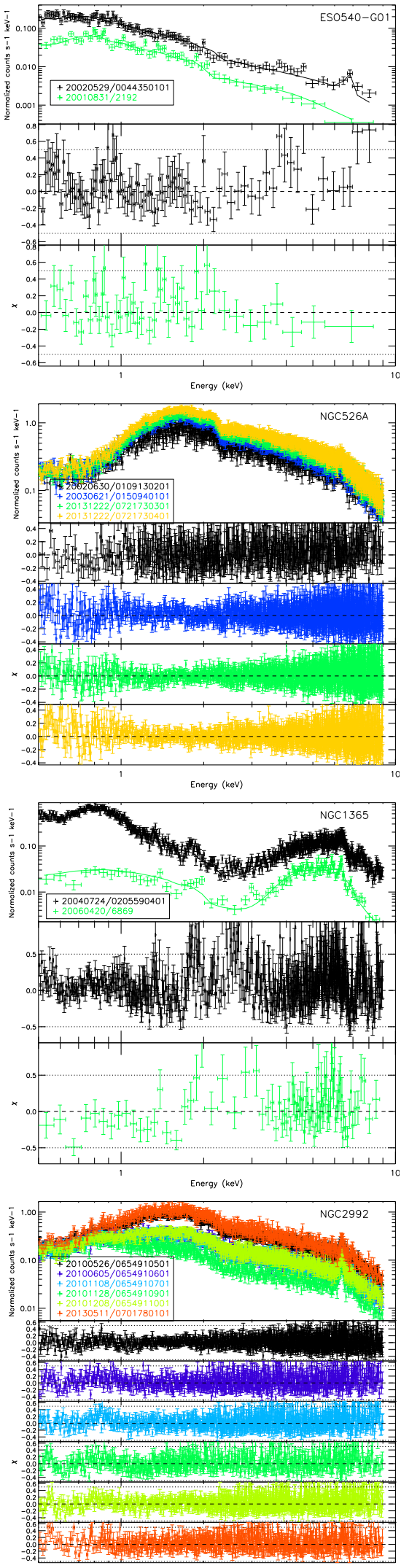

Eneroy (kein)
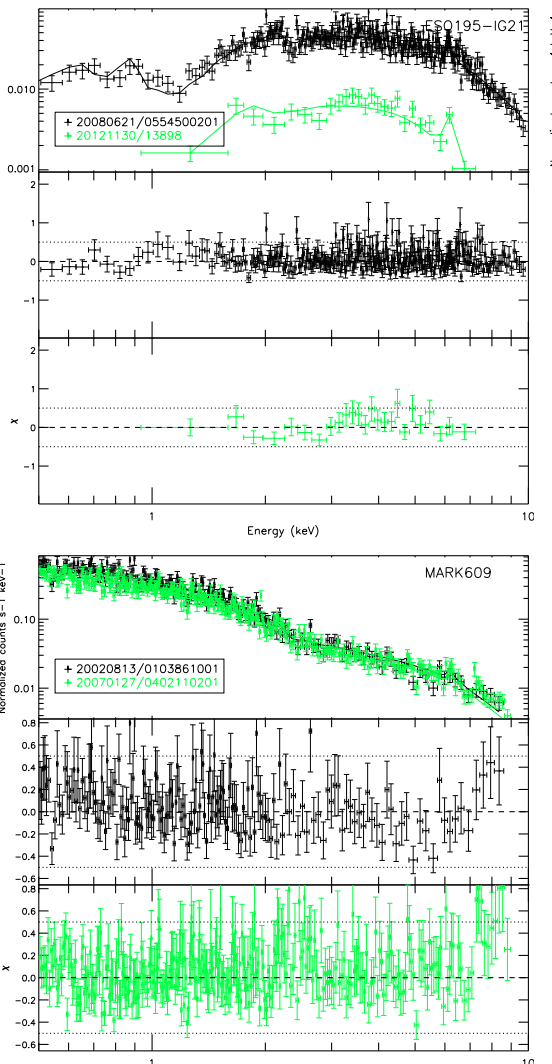

Energy (kel)
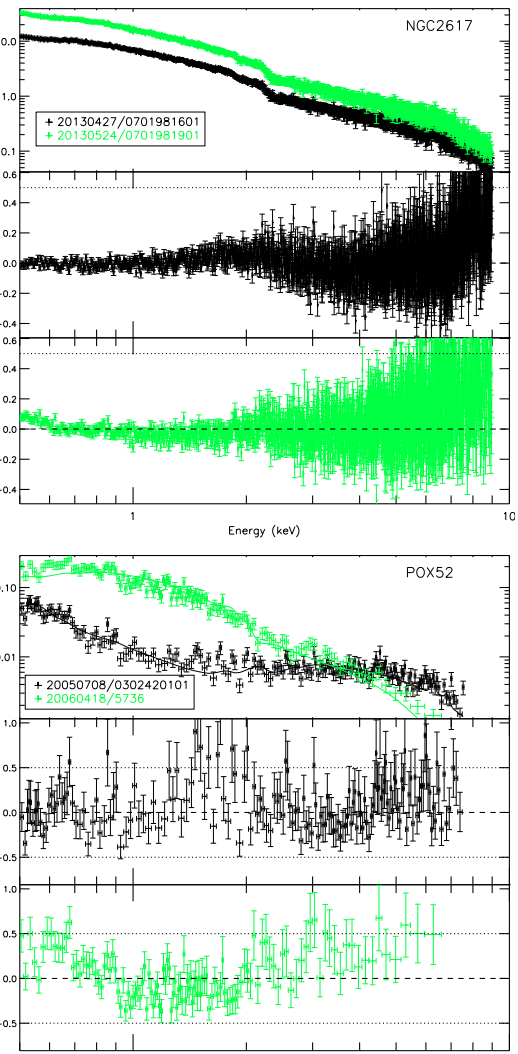

Eneray (kev)

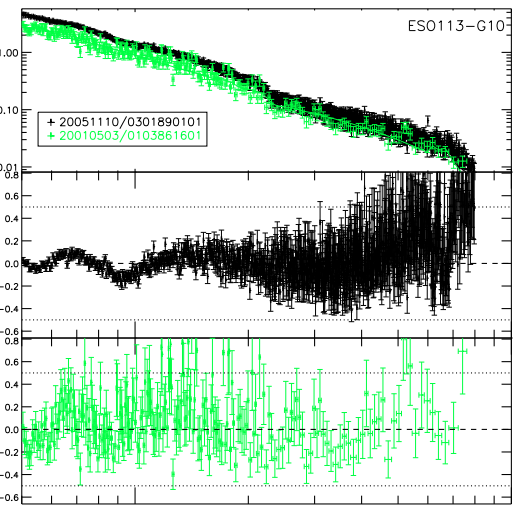

Eneray (kei)

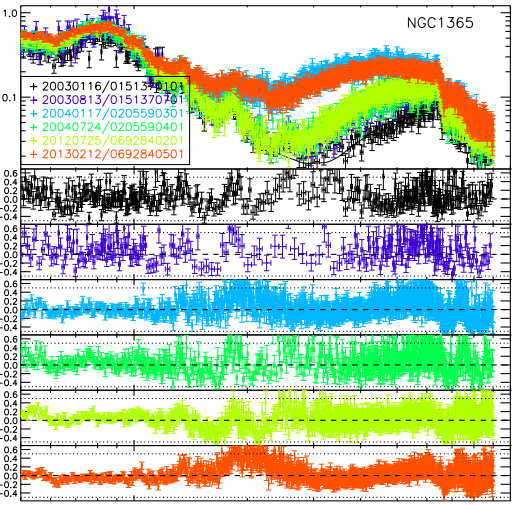

Energy (keV)
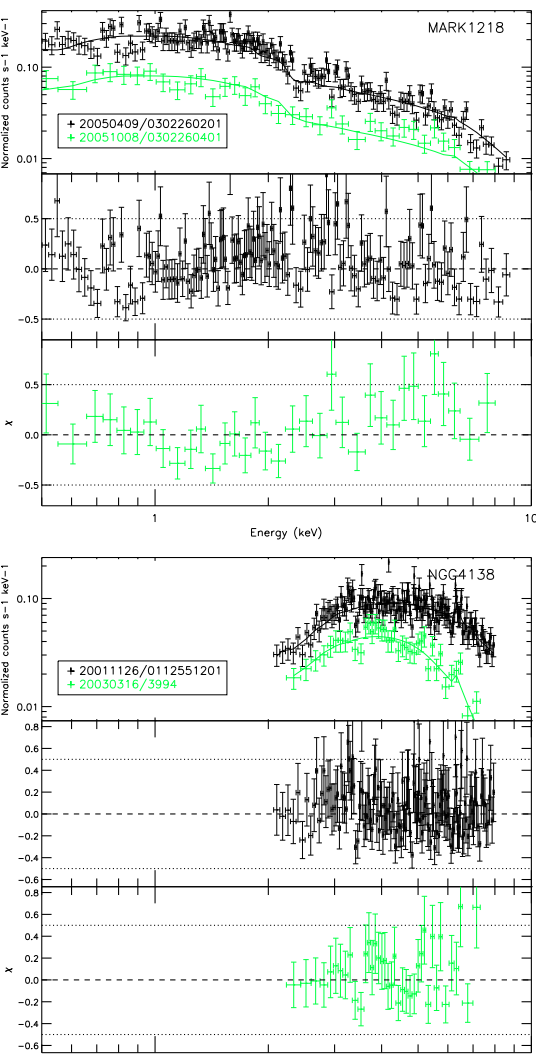

Eneray (keV)

Fig. 1. For each object, top: simultaneous fit of X-ray spectra; from second row on: residuals in units of $\sigma$. The legends contain the date (in the format yyyymmdd) and the obsID. Details are given in Table 1 . 

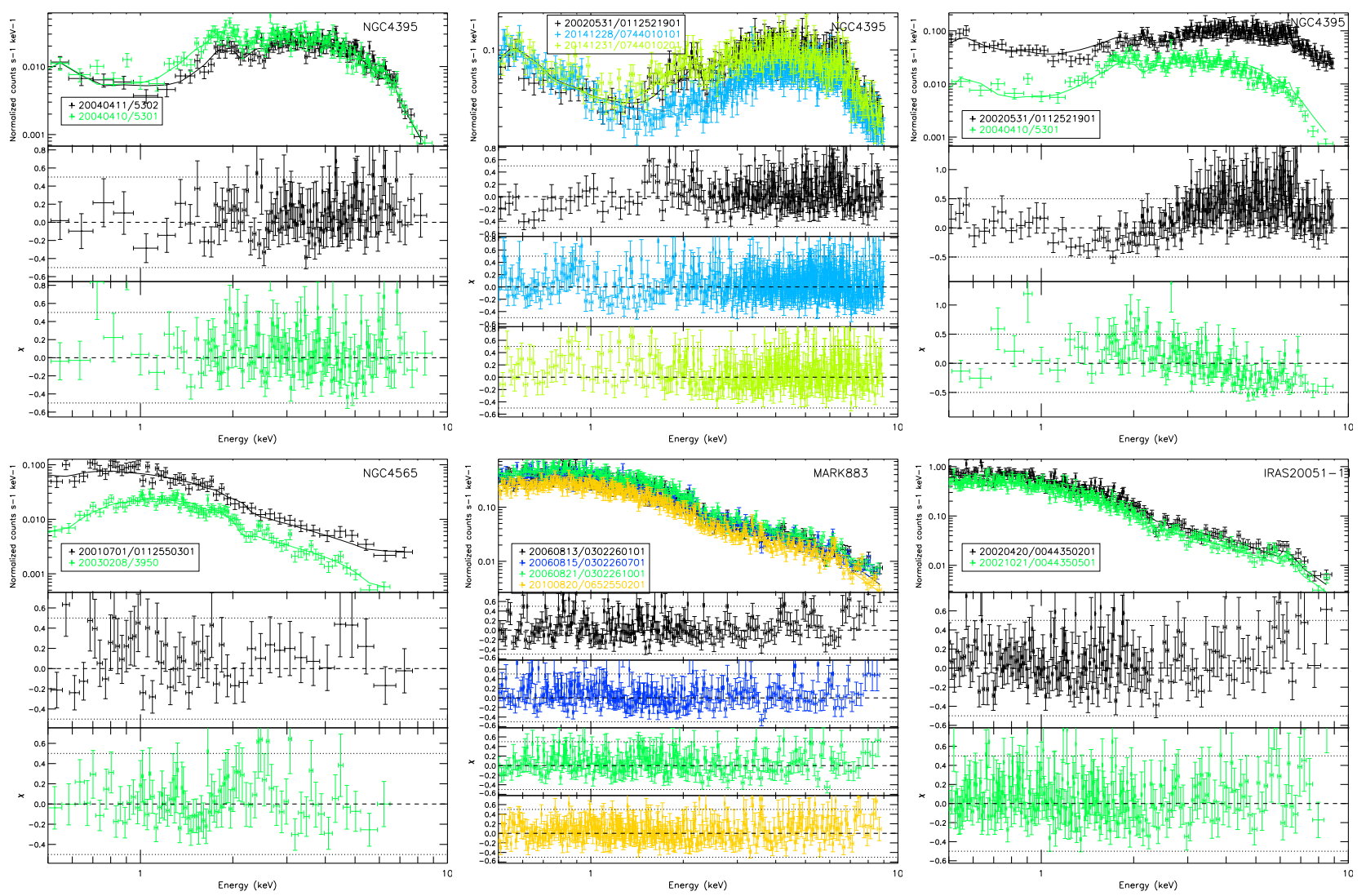

Fig. 1. continued.

and NGC 4395). Among these, all but NGC 1365 show variations above the $3 \sigma$ confidence level in the three energy bands. Among the eight observations analyzed for NGC 1365, four do not show variations in any band, two show variations in the total and hard bands, and another two show variations in the three energy bands (both from 2012). NGC 2992 shows variations in the total and hard energy bands. Five observations were analyzed for this source, two of them not showing variations in any of the bands. NGC 2617 shows variations in the total and soft energy bands, in both cases above the $3 \sigma$ confidence level.

\subsection{Long-term UV flux variability}

UV data are available for nine sources. The remaining six sources do not have more than one XMM-Newton observations or different filters were used for the observations and thus cannot be directly compared.

Among the nine sources, two of them do not show UV variability (MARK 1218 and IRAS 20051-1117), whereas the remaining seven sources show variations in at least one filter (see Table A.1 and Fig. A.1). We note that all the reported variations are above the $3 \sigma$ confidence level.

\section{Discussion}

The optical and X-ray spectra of Seyfert 1.8/1.9 resembles those of Seyfert 2. For this reason, many studies aiming at analyzing the properties of obscured sources have included all these nuclei in the same samples (e.g., Risaliti et al. 1999; Akylas \& Georgantopoulos 2009; Malizia et al. 2012). However, a study of their variability properties compared to those of Seyfert 2 represents a powerful tool in revealing similarities

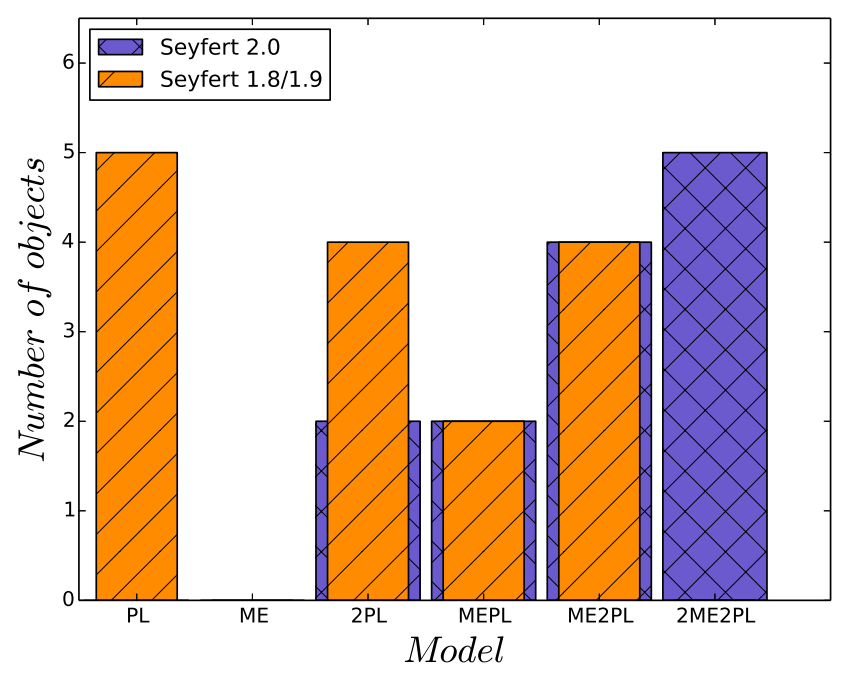

Fig. 2. Best fit models for Seyfert 1.8/1.9 and 2.

and differences. In this work we can compare these properties in an homogeneous way for the first time using the results for Seyfert 1.8/1.9 presented here and the sample of Seyfert 2 presented in Hernández-García et al. (2015).

\subsection{Spectral properties}

Figure 2 shows the models used for Seyfert 1.8/1.9 and 2. The main conclusion is that Seyfert 2 require, in general, more complex models to fit the data. The difference cannot be attributed to different spectral qualities since the average $\mathrm{S} / \mathrm{N}$ - which were estimated following Stoehr et al. (2008) - for each spectrum is 

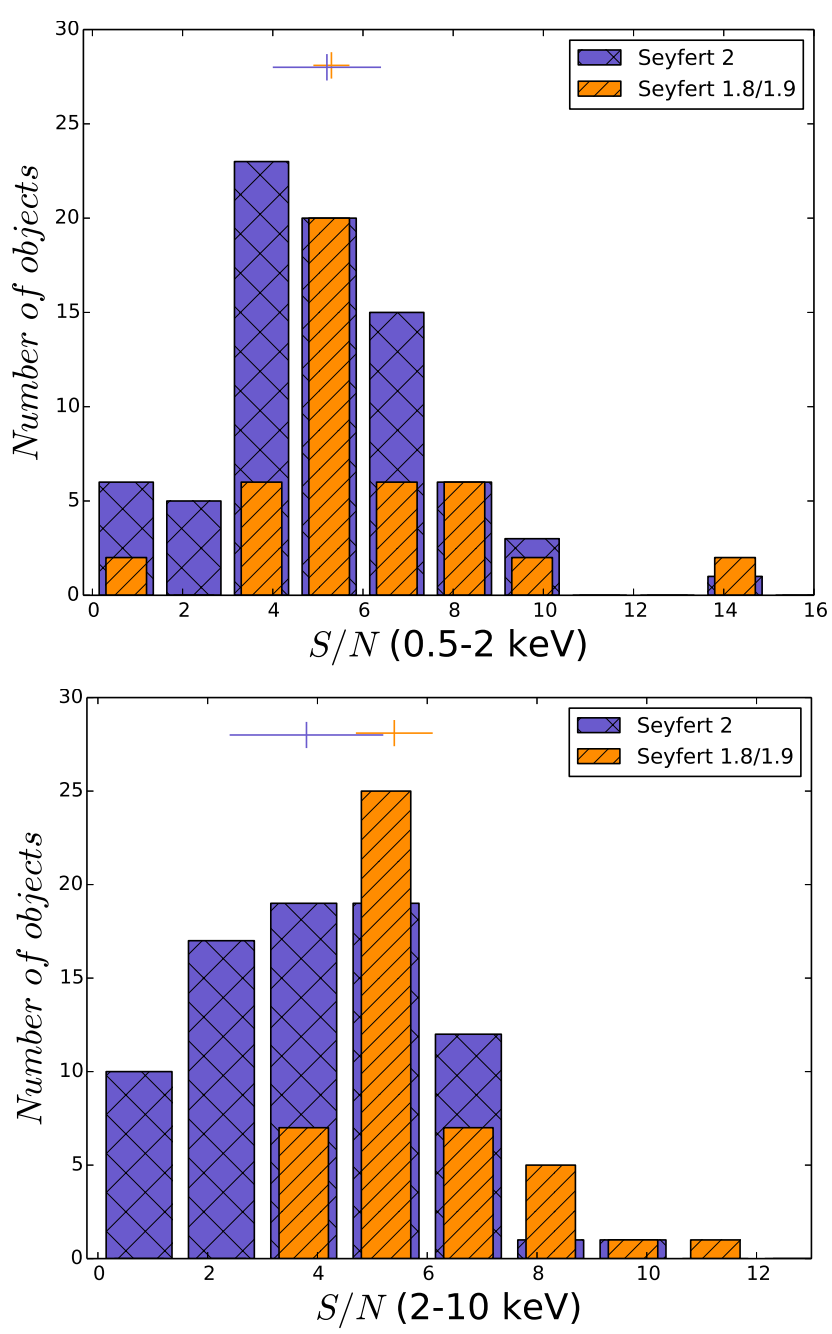

Fig. 3. $\mathrm{S} / \mathrm{N}$ ratio for the individual spectra of Seyfert $1.8 / 1.9$ and Seyfert 2 in the soft (0.5-2 keV, upper panel) and hard (2-10 keV, bottom panel) energy bands. The crosses represent the median values.

similar for both type 1.8/1.9 and type 2 samples in the $0.5-2 \mathrm{keV}$ and 2-10 keV energy bands (see Fig. 3). In Table 3, we show the median values $(25 \%$ and $75 \%$ percentiles) of the spectral parameters for the Seyfert 1.8/1.9 and 2 nuclei. It can be seen that the main difference between these AGN families is in $N_{\mathrm{H} 2}$ (see also Fig. 4), in agreement with previous studies (Risaliti et al. 1999; Guainazzi et al. 2001; Akylas \& Georgantopoulos 2009; Brightman \& Nandra 2011b). This is in fact the main reason why Seyfert 1.8/1.9 have been explained as nuclei that are less obscured than Seyfert 2, their difference being ascribed only to the absorbing material.

However, another difference is observed in the temperatures of the thermal emission, with Seyfert 2 having a bimodal distribution centered at $K T_{1} \sim 0.7 \mathrm{keV}$ and $K T_{2} \sim 0.2 \mathrm{keV}$ whereas Seyfert 1.8/1.9 show only one temperature regime centered at $K T \sim 0.2 \mathrm{keV}$. Moreover the comparison with the study by Brightman \& Nandra (2011a,b) on the $12 \mu \mathrm{m}$ complete sample of Seyferts show that a thermal component is fitted in Seyfert 11.5 (Seyfert 2) in 15 (24) cases, with 12 (6) of them centered at $0.1 \mathrm{keV}$ and three (18) centered at $0.7 \mathrm{keV}$. This result might indicate that the thermal component observed in Seyfert 1.8/1.9 is more similar to that observed in Seyfert 1 than to that in type 2.

In this context, it should be noticed that a more realistic physical model for the absorbing material in Seyfert 1.8/1.9 might be

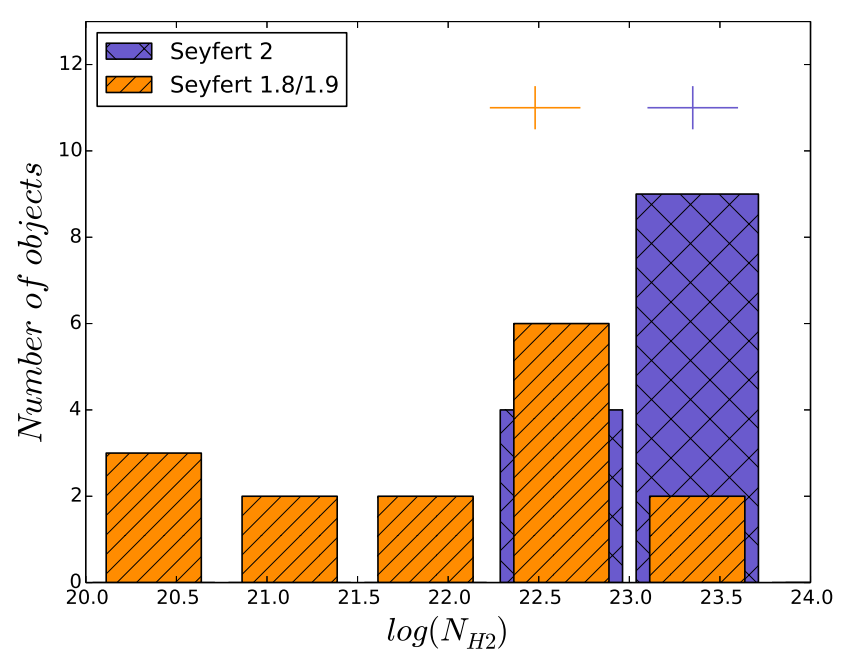

Fig. 4. Column density, $N_{\mathrm{H} 2}$, for Seyfert 1.8/1.9 and Seyfert 2 in logarithmic scale. The crosses represent the median values.

represented by ionized absorption (i.e., winds) instead of neutral absorption (i.e., the torus). We changed the neutral absorption by an ionized absorption in our models but the spectral fits were statistically the same (the fits did not improve at the $99.9 \%$ of confidence level using the $F$-test), except in the case of NGC 1365, where the presence of ionized absorption is well established (Risaliti et al. 2005a; Guainazzi et al. 2009). Since we cannot differentiate between neutral or ionized absorption due to the resolution of the spectra presented in this work, this issue should be explored using high spectral resolution data.

\subsection{Short-term variability}

Further information can be obtained from the analysis of the variability. X-ray short-term (i.e., intra-day) variations in Seyfert 2 galaxies were not detected in Hernández-García et al. (2015), and thus all the measurements of $\sigma_{\text {NXS }}^{2}$ are upper limits. It is interesting to notice that most Seyfert 1 show changes at these timescales (Nandra et al. 1997; Turner et al. 1997), but variations at these timescales in a few Seyfert 2 have also been reported in the literature (e.g., González-Martín \& Vaughan 2012). Even though the physical origin of these variations is not yet well settled, the involved timescales imply that they occur very close to the SMBH, and it has been suggested that accretion-disk and/or corona instabilities or variations in the accretion rate may be involved (Uttley et al. 2005; Breedt et al. 2010; McHardy 2010; Soldi et al. 2014). Figure 5 shows the $\sigma_{\text {NXS }}^{2}$ in the $2-10 \mathrm{keV}$ energy band against $M_{\mathrm{BH}}$ for the variable Seyfert 1.8/1.9 in our sample and the upper limits for the Seyfert 2 together with the results reported by Ponti et al. (2012) for the CAIXA's sample, which includes unobscured sources. For a proper comparison, we used their $\sigma_{\text {NXS }}^{2}$ calculated for light curve segments of $40 \mathrm{ks}$. The green squares represent those sources with a FWHM of the $\mathrm{H}_{\beta}$ larger than $2500 \mathrm{~km} \mathrm{~s}^{-1}$, the red triangles those with $F M W H\left(\mathrm{H}_{\beta}\right)<2500 \mathrm{~km} \mathrm{~s}^{-1}$, and the black hexagons the sources where they did not report the $\operatorname{FMWH}\left(\mathrm{H}_{\beta}\right)$. It has been shown that the $2-10 \mathrm{keV}$ power spectrum in AGN follows a power law of slope $\Gamma \sim-2$ at high frequencies, which then flattens to a slope of $\Gamma \sim-1$ below a break frequency (Papadakis 2004; O'Neill et al. 2005; Miniutti et al. 2009). This model is named the universal power spectrum density (PSD) model because the integral of the PSD is equal to the $\sigma_{\text {NXs }}^{2}$ of a light curve (Vaughan et al. 2003). The dotted line 


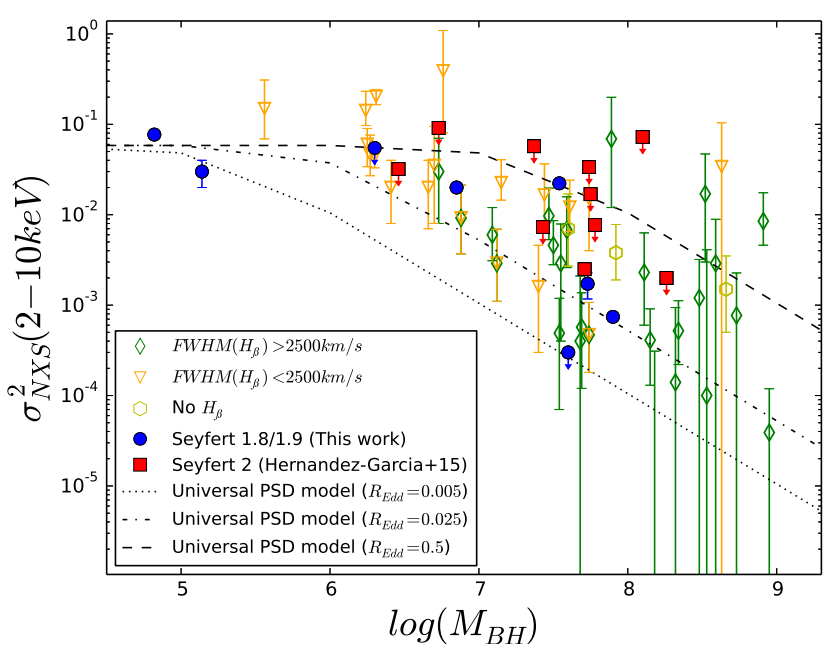

Fig. 5. Normalized excess variance, $\sigma_{\mathrm{NXS}}^{2}$, against the black hole mass, $M_{\mathrm{BH}}$ for the Seyfert 1.8/1.9 in the sample (blue circles) and Seyfert 2 in Hernández-García et al. (2015; red squares). Results for the CAIXA sample using light curve segments of $40 \mathrm{ks}$ (Ponti et al. 2012) are presented, including sources with $F M W H\left(\mathrm{H}_{\beta}\right)>2500 \mathrm{~km} \mathrm{~s}^{-1}$ (green diamonds), $F M W H\left(\mathrm{H}_{\beta}\right)<2500 \mathrm{~km} \mathrm{~s}^{-1}$ (orange triangles), and those with no $\mathrm{H}_{\beta}$ (yellow hexagons). The dotted, dash-dotted, and dashed lines are the universal PSD model, represented as a broken power law (Papadakis 2004) using $R_{\text {Edd }}=[0.005,0.025,0.5]$.

in Fig. 5 represents this model as in Papadakis (2004, see also González-Martín et al. 2011), which was obtained for a sample of broad line Seyfert 1. For the relation, we used an Eddington ratio of $R_{\text {Edd }}=[0.005,0.025,0.5]$, which is represented as a dotted, dash-dotted, and dashed lines in Fig. 5, respectively. It can be observed that the Seyfert 1.8/1.9 in our sample fit well both with this model and with the results reported by Ponti et al. (2012), as well as Seyfert 2 do. Thereof the short-term variability in Seyfert 1, Seyfert 1.8/1.9 and Seyfert 2 is consistent within the statistical uncertainties and thus we cannot provide evidence for a difference among the classes in this respect until the variations in Seyfert 2 are rejected.

\subsection{Long-term variations}

We found differences in the spectral variations between Seyfert 1.8/1.9 and Seyfert 2 in timescales from weeks to years. Variations at soft energies are only detected in Seyfert 1.8/1.9. The origin of the soft X-ray emission in Seyferts is under debate, maybe related to e.g., the possible dilution effect of scattering by photoionized gas (e.g., González-Martín et al. 2010) or to a warm absorber (WA), consisting on ionized absorbing gas, that is observed in about 65\% of Seyfert 1s (Laha et al. 2014), and it is highly variable (e.g., Winter et al. 2012; Laha et al. 2016, and references therein). However, the exposure times of the high resolution data (e.g., from the Reflection Grating Spectrometer) of the sources discussed in this paper are too short to constrain the properties of the soft X-ray emission, and thus the nature of the soft X-ray variations (e.g., Laha et al. 2011; Giustini et al. 2017).

At long timescales we find that Seyfert 1.8/1.9, as well as Seyfert 2 and LINERs, show hard X-ray variations in timescales between months and years, these changes being related to intrinsic variations of the hard nuclear continuum. This suggests that the emission mechanism is the same in these AGN families. We have also computed the variability dynamical timescale against the time between the observations for the two families. We did not find differences in the variability of these sources, neither any obvious trend against the time difference. We note that a caveat in our analysis might be that the observations were taken at random epochs, and thus the timescale between the observations do not refer to the variability timescales.

The AGN continuum at X-rays comes from the Comptonization of photons from the inner parts of the accretion disk (Shakura \& Sunyaev 1973). Thus, these long-term variations might be related to fluctuations in the inner accretion disk (e.g., Hernández-García et al. 2016). Moreover, variations due to absorbing clouds (i.e., $N_{\mathrm{H}}$ ) are common in our sample, both in Seyfert 1.8/1.9 (six out of the 15, i.e., 40\%) and 2 s (four out of the 11 variable sources, i.e., 36\%). However, these kind of eclipses are also observed in Seyfert 1s (Risaliti et al. 2011; Sanfrutos et al. 2013; Markowitz et al. 2014; Agís-González et al. 2014). When it is possible to estimate the cloud velocity, and thus the location of the absorbing material, the clouds appear to be located very close to the BLR or within the borderline between the BLR and the torus (Risaliti et al. 2009, 2013; Nardini \& Risaliti 2011; Walton et al. 2014; Connolly et al. 2014; Markowitz et al. 2014; Parker et al. 2015; Giustini et al. 2017). The velocities of the clouds in Seyfert 1.8/1.9 (NGC 1365, NGC 2617, NGC 2992, and NGC 4395) and Seyfert 2 (Mark 1210, and NGC 4507) in our sample are greater than $10^{3} \mathrm{~km} \mathrm{~s}^{-1}$ (following the procedure in Risaliti et al. 2010) ${ }^{9}$. Therefore, these eclipses due to clouds passing through our line of sight seem to be happening at distances from the accretion disk consistent within or very close to the location of the BLR.

Furthermore, while variations at UV frequencies are not detected in Seyfert 2, these changes are observed in Seyfert 1.8/1.9 in the present work. The same behavior is also seen in Seyfert $1 \mathrm{~s}$ (e.g., Cardaci 2010; Netzer 2013). This result might indicate a less obstructed view of the accretion disk in Seyfert 1.8/1.9 compared to that of Seyfert 2, in agreement with the higher values of $N_{\mathrm{H} 2}$ in Seyfert 2 compared to those of Seyfert 1.8/1.9.

As a final remark, we would like to warn the reader of the simplistic association of Seyfert 1.8/1.9 to Seyfert 2, since different variability properties are observed in the two AGN groups both at X-ray and at UV frequencies. We leave open the possibility that Seyfert 1.8/1.9 behaves more like Seyfert 1, but a proper, systematic, homogeneous comparison between the properties of these families is necessary.

\section{Summary and conclusions}

We have performed a spectral and variability analysis at X-ray and UV frequencies of a sample of fifteen galaxies classified as Seyfert 1.8/1.9 based on optical spectroscopy. The main results of the study can be summarized as follows:

- X-ray long-term variability is found in all the 15 nuclei. None of the sources are classified as Compton-thick candidates, and two of them have been classified as changing-look candidates.

- The main variability pattern is related to intrinsic changes in the sources, which are observed in ten nuclei. Changes in the column density are also frequent, as they are observed in six nuclei. Variations at soft energies are detected in six sources.

- X-ray short-term variations are detected in six out of the eight studied sources.

\footnotetext{
9 Since the timescales between the observations were obtained at random, we can only estimate its velocities when the timescale is shorter than approximately one month.
} 
- Variations at UV frequencies are detected in seven out of the nine sources where data were available.

We have compared the properties of Seyfert 1.8/1.9 with the results of a sample of Seyfert 2 that were analyzed using the same methodology applied in this work (Hernández-García et al. 2015), allowing a homogeneous comparison. The main conclusions obtained from this work are the following:

- The X-ray long term variations occur in a similar way in all the nuclei and are mainly related to intrinsic changes of the nuclear continuum. Variations in the absorbing column densities are also frequent in both AGN families.

- X-ray short-term, soft X-ray, and UV long term variations are detected in Seyfert 1.8/1.9 but not in Seyfert 2, indicating that the view of the SMBH is unobstructed in Seyfert 1.8/1.9 and obstructed in Seyfert 2.

- We caution on the simplistic association of Seyfert 1.8/1.9 to Seyfert 2 to classify all of them as obscured sources, because they show different variability properties at X-ray and UV frequencies.

A similar study of a sample of Seyfert 1 would be required in order to homogeneously compare their properties and to test whether Seyfert 1.8/1.9 are similar to these AGN or not.

Acknowledgements. We thank the anonymous referee for helpful comments We acknowledge the AGN group at the IAA for helpful comments during this work. This work was financed by MINECO grant AYA 2010-15169, AYA 2013 42227-P, and Junta de Andalucía TIC114. O.G.M. acknowledges financial support from the UNAM PAPIIT IA100516 project. L.H.G. and F.P. acknowledge the ASI/INAF agreement number 2013-023-R1. This research made use of data obtained from the Chandra Data Archive provided by the Chandra X-ray Center (CXC). This research made use of data obtained from the XMM-Newton Data Archive provided by the XMM-Newton Science Archive (XSA). This research made use of the NASA/IPAC extragalactic database (NED), which is operated by the Jet Propulsion Laboratory under contract with the National Aeronautics and Space Administration. We acknowledge the usage of the HyperLeda data base (http: //leda.univ-lyon $1 . \mathrm{fr}$ )

\section{References}

Agís-González, B., Miniutti, G., Kara, E., et al. 2014, MNRAS, 443, 2862 Akylas, A., \& Georgantopoulos, I. 2009, A\&A, 500, 999 Antonucci, R. 1993, ARA\&A, 31, 473

Barth, A. J., Ho, L. C., Rutledge, R. E., \& Sargent, W. L. W. 2004, ApJ, 607, 90 Bassani, L., Dadina, M., Maiolino, R., et al. 1999, ApJS, 121, 473 Baumgartner, W. H., Tueller, J., \& Mushotzky, R. F. 2008, ATel, 1794 Beckmann, V., Gehrels, N., \& Tueller, J. 2007, ApJ, 666, 122 Benítez, E., Méndez-Abreu, J., Fuentes-Carrera, I., et al. 2013, ApJ, 763, 136 Braito, V., Ballo, L., Reeves, J. N., et al. 2013, MNRAS, 428, 2516 Braito, V., Reeves, J. N., Gofford, J., et al. 2014, ApJ, 795, 87 Breedt, E., McHardy, I. M., Arévalo, P., et al. 2010, MNRAS, 403, 605 Brenneman, L. W., Risaliti, G., Elvis, M., \& Nardini, E. 2013, MNRAS, 429, 2662

Brightman, M., \& Nandra, K. 2011a, MNRAS, 413, 1206

Brightman, M., \& Nandra, K. 2011b, MNRAS, 414, 3084

Cackett, E. M., Fabian, A. C., Zogbhi, A., et al. 2013, ApJ, 764, L9

Cameron, D. T., McHardy, I., Dwelly, T., et al. 2012, MNRAS, 422, 902

Cappi, M., Panessa, F., Bassani, L., et al. 2006, A\&A, 446, 459

Cardaci, M. V. 2010, Ph.D. Thesis, Universidad Autónoma de Madrid

Cohen, R. D., Puetter, R. C., Rudy, R. J., Ake, T. B., \& Foltz, C. B. 1986, ApJ 311,135

Condon, J. J., Condon, M. A., Gisler, G., \& Puschell, J. J. 1982, ApJ, 252, 102

Condon, J. J., Yin, Q. F., Thuan, T. X., \& Boller, T. 1998, AJ, 116, 2682

Connolly, S. D., McHardy, I. M., \& Dwelly, T. 2014, MNRAS, 440, 3503

Coziol, R., Demers, S., Pena, M., et al. 1993, AJ, 105, 35

Dahari, O., \& De Robertis, M. M. 1988, ApJS, 67, 249

Dewangan, G. C., Mathur, S., Griffiths, R. E., \& Rao, A. R. 2008, ApJ, 689, 762

Dickey, J. M., \& Lockman, F. J. 1990, ARA\&A, 28, 215
Eracleous, M., Hwang, J. A., \& Flohic, H. M. L. G. 2010, ApJ, 711, 796 Fabian, A. C., Lohfink, A., Kara, E., et al. 2015, MNRAS, 451, 4375 Garmire, G. P., Bautz, M. W., Ford, P. G., Nousek, J. A., \& Ricker, J. G. R. 2003, in SPIE Conf. Ser. 4851, eds. J. E. Truemper, \& H. D. Tananbaum, 28 Georgantopoulos, I., Zezas, A., \& Ward, M. J. 2003, ApJ, 584, 129 Georgantopoulos, I., Papadakis, I., Zezas, A., \& Ward, M. J. 2004, ApJ, 614, 634 Ghisellini, G., Haardt, F., \& Matt, G. 1994, MNRAS, 267, 743

Gilli, R., Maiolino, R., Marconi, A., et al. 2000, A\&A, 355, 485

Giustini, M., Costantini, E., De Marco, B., et al. 2017, A\&A, 597, A66

Glass, I. S. 1997, MNRAS, 292, L50

González-Martín, O., \& Vaughan, S. 2012, A\&A, 544, A80

González-Martín, O., Acosta-Pulido, J. A., Perez Garcia, A. M., \& Ramos Almeida, C. 2010, ApJ, 723, 1748

González-Martín, O., Papadakis, I., Reig, P., \& Zezas, A. 2011, A\&A, 526, A132 Goodrich, R. W. 1995, ApJ, 440, 141

Griffiths, R. E., Schwartz, D. A., Schwarz, J., et al. 1979, ApJ, 230, L21

Guainazzi, M., Fiore, F., Matt, G., \& Perola, G. C. 2001, MNRAS, 327, 323

Guainazzi, M., Fabian, A. C., Iwasawa, K., Matt, G., \& Fiore, F. 2005, MNRAS, 356,295

Guainazzi, M., Risaliti, G., Nucita, A., et al. 2009, A\&A, 505, 589

Hernández-García, L., González-Martín, O., Márquez, I., \& Masegosa, J. 2013, A\&A, 556, A47

Hernández-García, L., González-Martín, O., Masegosa, J., \& Márquez, I. 2014 A\&A, 569, A26

Hernández-García, L., Masegosa, J., González-Martín, O., \& Márquez, I. 2015a, A\&A, 579, A90

Hernández-García, L., Vaughan, S., Roberts, T. P., \& Middleton, M. 2015b, MNRAS, 453, 2877

Hernández-García, L., Masegosa, J., González-Martín, O., Márquez, I., \& Perea, J. 2016, ApJ, 824, 7

Hickson, P., Kindl, E., \& Auman, J. R. 1989, ApJS, 70, 687

Ho, L. C., \& Ulvestad, J. S. 2001, ApJS, 133, 77

Ho, L. C., Filippenko, A. V., Sargent, W. L. W., \& Peng, C. Y. 1997, ApJS, 112, 391

Jorsater, S., \& van Moorsel, G. A. 1995, AJ, 110, 2037

Kalberla, P. M. W., Burton, W. B., Hartmann, D., et al. 2005, A\&A, 440, 775

Kara, E., Zoghbi, A., Marinucci, A., et al. 2015, MNRAS, 446, 737

Laha, S., Dewangan, G. C., \& Kembhavi, A. K. 2011, ApJ, 734, 75

Laha, S., Guainazzi, M., Dewangan, G. C., Chakravorty, S., \& Kembhavi, A. K. 2014, MNRAS, 441, 2613

Laha, S., Guainazzi, M., Chakravorty, S., Dewangan, G. C., \& Kembhavi, A. K. 2016, MNRAS, 457, 3896

LaMassa, S. M., Heckman, T. M., Ptak, A., et al. 2011, ApJ, 729, 52

LaMassa, S. M., Yaqoob, T., Ptak, A. F., et al. 2014, ApJ, 787, 61

Lawrence, A., Watson, M. G., Pounds, K. A., \& Elvis, M. 1987, Nature, 325, 694

Maiolino, R., \& Rieke, G. H. 1995, ApJ, 454, 95

Maiolino, R., Salvati, M., Bassani, L., et al. 1998, A\&A, 338, 781

Malizia, A., Bassani, L., Bazzano, A., et al. 2012, MNRAS, 426, 1750

Markowitz, A. G., Krumpe, M., \& Nikutta, R. 2014, MNRAS, 439, 1403

Marquez, I., \& Moles, M. 1996, A\&AS, 120, 1

Matt, G., Guainazzi, M., \& Maiolino, R. 2003, MNRAS, 342, 422

McHardy, I. 2010, in Lect. Notes Phys. 794, ed. T. Belloni (Berlin: Springer Verlag), 203

McHardy, I. M., Connolly, S. D., Peterson, B. M., et al. 2016, Astron. Nachr., 337,500

Minezaki, T., Yoshii, Y., Kobayashi, Y., et al. 2006, ApJ, 643, L5

Miniutti, G., Ponti, G., Greene, J. E., et al. 2009, MNRAS, 394, 443

Moran, E. C., Halpern, J. P., \& Helfand, D. J. 1996, ApJS, 106, 341

Moran, E. C., Barth, A. J., Kay, L. E., \& Filippenko, A. V. 2000, ApJ, 540, L73

Moran, E. C., Eracleous, M., Leighly, K. M., et al. 2005, AJ, 129, 2108

Mulchaey, J. S., Wilson, A. S., \& Tsvetanov, Z. 1996, ApJS, 102, 309

Murphy, K. D., Yaqoob, T., \& Terashima, Y. 2007, ApJ, 666, 96

Mushotzky, R. F. 1982, ApJ, 256, 92

Nagar, N. M., Wilson, A. S., Mulchaey, J. S., \& Gallimore, J. F. 1999, ApJS, 120, 209

Nagar, N. M., Falcke, H., Wilson, A. S., \& Ulvestad, J. S. 2002, A\&A, 392, 53

Nandra, K., George, I. M., Mushotzky, R. F., Turner, T. J., \& Yaqoob, T. 1997, ApJ, 476, 70

Nardini, E., \& Risaliti, G. 2011, MNRAS, 417, 2571

Netzer, H. 2013, The Physics and Evolution of Active Galactic Nuclei (Cambridge, UK: Cambridge University Press)

O’Neill, P. M., Nandra, K., Papadakis, I. E., \& Turner, T. J. 2005, MNRAS, 358, 1405

O’Neill, P. M., Kaspi, S., Laor, A., et al. 2006, ApJ, 645, 160

Osterbrock, D. E. 1977, ApJ, 215, 733

Osterbrock, D. E. 1981, ApJ, 249, 462

Osterbrock, D. E., \& Dahari, O. 1983, ApJ, 273, 478 
Osterbrock, D. E., \& Martel, A. 1993, ApJ, 414, 552

Panessa, F., \& Bassani, L. 2002, A\&A, 394, 435

Panessa, F., Wolter, A., Pellegrini, S., et al. 2005, ApJ, 631, 707

Panessa, F., Bassani, L., Cappi, M., et al. 2006, A\&A, 455, 173

Papadakis, I. E. 2004, MNRAS, 348, 207

Parker, M. L., Walton, D. J., Fabian, A. C., \& Risaliti, G. 2014, MNRAS, 441, 1817

Parker, M. L., Fabian, A. C., Matt, G., et al. 2015, MNRAS, 447, 72

Peterson, B. M. 1997, An Introduction to Active Galactic Nuclei (Cambridge University Press)

Pietsch, W., Bischoff, K., Boller, T., et al. 1998, A\&A, 333, 48

Polletta, M., Bassani, L., Malaguti, G., Palumbo, G. G. C., \& Caroli, E. 1996 , ApJS, 106, 399

Ponti, G., Papadakis, I., Bianchi, S., et al. 2012, A\&A, 542, A83

Porquet, D., Uttley, P., Reeves, J. N., et al. 2007, A\&A, 473, 67

Rees, M. J. 1984, ARA\&A, 22, 471

Risaliti, G. 2002, A\&A, 386, 379

Risaliti, G., Maiolino, R., \& Salvati, M. 1999, ApJ, 522, 157

Risaliti, G., Elvis, M., \& Nicastro, F. 2002, ApJ, 571, 234

Risaliti, G., Bianchi, S., Matt, G., et al. 2005a, ApJ, 630, L129

Risaliti, G., Elvis, M., Fabbiano, G., Baldi, A., \& Zezas, A. 2005b, ApJ, 623, L93

Risaliti, G., Elvis, M., Fabbiano, G., et al. 2007, ApJ, 659, L111

Risaliti, G., Miniutti, G., Elvis, M., et al. 2009, ApJ, 696, 160

Risaliti, G., Elvis, M., Bianchi, S., \& Matt, G. 2010, MNRAS, 406, L20

Risaliti, G., Nardini, E., Salvati, M., et al. 2011, MNRAS, 410, 1027

Risaliti, G., Harrison, F. A., Madsen, K. K., et al. 2013, Nature, 494, 449

Rivers, E., Risaliti, G., Walton, D. J., et al. 2015, ApJ, 804, 107

Sanfrutos, M., Miniutti, G., Agís-González, B., et al. 2013, MNRAS, 436, 1588

Shakura, N. I., \& Sunyaev, R. A. 1973, A\&A, 24, 337

Shappee, B. J., Prieto, J. L., Grupe, D., et al. 2014, ApJ, 788, 48

Shi, Y., Rieke, G. H., Smith, P., et al. 2010, ApJ, 714, 115

Shu, X. W., Yaqoob, T., Murphy, K. D., et al. 2010, ApJ, 713, 1256

Singh, V., Shastri, P., \& Risaliti, G. 2011, A\&A, 532, A84

Smith, J. E., Robinson, A., Alexander, D. M., et al. 2004, MNRAS, 350, 140

Soldi, S., Beckmann, V., Baumgartner, W. H., et al. 2014, A\&A, 563, A57
Stoehr, F., White, R., Smith, M., et al. 2008, in Astronomical Data Analysis Software and Systems XVII, eds. R. W. Argyle, P. S. Bunclark, \& J. R. Lewis, ASP Conf. Ser., 394, 505

Strüder, L., Briel, U., Dennerl, K., et al. 2001, A\&A, 365, L18

Terashima, Y., \& Wilson, A. S. 2003, ApJ, 583, 145

Thornton, C. E., Barth, A. J., Ho, L. C., Rutledge, R. E., \& Greene, J. E. 2008, ApJ, 686, 892

Tremaine, S., Gebhardt, K., Bender, R., et al. 2002, ApJ, 574, 740

Trippe, M. L., Crenshaw, D. M., Deo, R., \& Dietrich, M. 2008, AJ, 135, 2048

Trippe, M. L., Crenshaw, D. M., Deo, R. P., et al. 2010, ApJ, 725, 1749

Trippe, M. L., Reynolds, C. S., Koss, M., Mushotzky, R. F., \& Winter, L. M. 2011, ApJ, 736, 81

Turner, T. J., George, I. M., Nandra, K., \& Mushotzky, R. F. 1997, ApJS, 113, 23

Turner, T. J., \& Pounds, K. A. 1989, MNRAS, 240, 833

Ulvestad, J. S. 1986, ApJ, 310, 136

Ulvestad, J. S., \& Wilson, A. S. 1984, ApJ, 278, 544

Urry, C. M., \& Padovani, P. 1995, PASP, 107, 803

Uttley, P. 2007, in The Central Engine of Active Galactic Nuclei, eds. L. C. Ho, \& J.-W. Wang, ASP Conf. Ser., 373, 149

Uttley, P., McHardy, I. M., \& Vaughan, S. 2005, MNRAS, 359, 345

Vasudevan, R. V., \& Fabian, A. C. 2009, MNRAS, 392, 1124

Vaughan, S., Edelson, R., Warwick, R. S., \& Uttley, P. 2003, MNRAS, 345, 1271

Vaughan, S., Iwasawa, K., Fabian, A. C., \& Hayashida, K. 2005, MNRAS, 356, 524

Véron-Cetty, M.-P., \& Véron, P. 2010, A\&A, 518, A10

Walton, D. J., Risaliti, G., Harrison, F. A., et al. 2014, ApJ, 788, 76

Wang, J.-M., \& Zhang, E.-P. 2007, ApJ, 660, 1072

Ward, M., Penston, M. V., Blades, J. C., \& Turtle, A. J. 1980, MNRAS, 193, 563

Whittle, M. 1992, ApJS, 79, 49

Winkler, H. 1992, MNRAS, 257, 677

Winter, L. M., Veilleux, S., McKernan, B., \& Kallman, T. R. 2012, ApJ, 745, 107

Wrobel, J. M., \& Ho, L. C. 2006, ApJ, 646, L95

Zdziarski, A. A., Johnson, W. N., Done, C., Smith, D., \& McNaron-Brown, K. 1995, ApJ, 438, L63

Zhang, Y.-H. 2011, ApJ, 726, 21 


\section{Appendix A: Additional material}

Table A.1. Observational details.

\begin{tabular}{|c|c|c|c|c|c|c|c|c|}
\hline Name & Instrument & ObsID & Date & $\begin{array}{c}R \\
\left({ }^{\prime \prime}\right)\end{array}$ & $\begin{array}{c}\text { Net exptime } \\
\text { (ks) }\end{array}$ & Counts & OM filter & $\begin{array}{c}\log \left(L_{\mathrm{UV}}\right) \\
(\mathrm{erg} / \mathrm{s})\end{array}$ \\
\hline$(1)$ & $(2)$ & $(3)$ & $(4)$ & $(5)$ & $(6)$ & $(7)$ & $(8)$ & $(9)$ \\
\hline \multirow[t]{3}{*}{ ESO 540-G01 } & Chandra $^{c}$ & 2192 & 2001-08-31 & 3 & 20 & 1316 & - & \\
\hline & XMM-Newton ${ }^{c}$ & 0044350101 & 2002-05-29 & 25 & 11 & 2728 & $U V W 2$ & $43.26_{-}^{+} 0.04$ \\
\hline & & & & & & & $U V W 1$ & $43.41_{-}^{+} 0.01$ \\
\hline \multirow[t]{2}{*}{ ESO 195-IG21 } & Chandra ${ }^{c}$ & 13898 & 2012-11-30 & 2 & 20 & 529 & - & \\
\hline & XMM-Newton ${ }^{c}$ & 0554500201 & 2008-06-21 & 20 & 25 & 6165 & & Not detected \\
\hline \multirow[t]{4}{*}{ ESO 113-G10 } & XMM-Newton & 0103861601 & 2001-05-03 & 25 & 4 & 10419 & $U V W 2$ & $42.68_{-}^{+} 0.06$ \\
\hline & XMM-Newton & 0301890101 & 2005-11-10 & 25 & 77 & 304379 & $U V W 2$ & $42.87_{-}^{+} 0.02$ \\
\hline & & & & & & & $U V M 2$ & $42.94_{-}^{+} 0.01$ \\
\hline & & & & & & & $U V W 1$ & $43.02_{-}^{+} 0.01$ \\
\hline \multirow[t]{8}{*}{ NGC 526A } & XMM-Newton ${ }^{c}$ & 0109130201 & 2002-06-30 & 35 & 8 & 20949 & $U V M 2$ & $42.13_{-}^{+} 0.08$ \\
\hline & & & & & & & $U V W 1$ & $42.27_{-}^{+} 0.03$ \\
\hline & $X M M-N e w t o n$ & 0150940101 & 2003-06-21 & 35 & 35 & 118532 & $U V W 2$ & $41.99_{-}^{+} 0.07$ \\
\hline & & & & & & & $U V M 2$ & $42.15_{-}^{+} 0.04$ \\
\hline & & & & & & & $U V W 1$ & $42.41_{-}^{+} 0.01$ \\
\hline & $X M M-N e w t o n$ & 0721730301 & 2013-12-22 & 35 & 48 & 177241 & - & \\
\hline & XMM-Newton & 0721730401 & $2013-12-22$ & 35 & 37 & 164811 & - & \\
\hline & Chandra $^{c}$ & 342 & 2000-02-07 & 4 & 9 & 1158 & - & \\
\hline \multirow[t]{2}{*}{ MARK 609} & XMM-Newton & 0103861001 & 2002-08-13 & 20 & 7 & 5054 & $U V W 2$ & $43.32_{-}^{+} 0.04$ \\
\hline & XMM-Newton & 0402110201 & 2007-01-27 & 20 & 16 & 10002 & $U V M 2$ & $43.37_{-}^{+} 0.01$ \\
\hline \multirow[t]{17}{*}{ NGC 1365} & Chandra $^{c}$ & 6869 & 2006-04-20 & 2 & 15 & 2021 & - & \\
\hline & XMM-Newton & 0151370101 & 2003-01-16 & 20 & 14 & 10599 & $U V W 2$ & $41.71_{-}^{+} 0.02$ \\
\hline & XMM-Newton & 0151370201 & 2003-02-09 & 20 & 3 & 1481 & - & \\
\hline & XMM-Newton & 0151370701 & 2003-08-13 & 20 & 6 & 6337 & $U V W 2$ & $41.71_{-}^{+} 0.03$ \\
\hline & $X M M-N e w t o n$ & 0205590301 & 2004-01-17 & 20 & 50 & 83303 & $U V W 1$ & $41.69_{-}^{+} 0.01$ \\
\hline & & & & & & & $U V M 2$ & $41.30_{-}^{+} 0.02$ \\
\hline & XMM-Newton ${ }^{c}$ & 0205590401 & 2004-07-24 & 20 & 29 & 30181 & $U V W 1$ & $42.21_{-}^{+} 0.01$ \\
\hline & & & & & & & $U V M 2$ & $41.95_{-}^{+} 0.01$ \\
\hline & $X M M-N e w t o n$ & 0505140201 & 2007-06-30 & 20 & 42 & 26349 & $U V W 1$ & $42.07_{-}^{+} 0.01$ \\
\hline & & & & & & & $U V M 2$ & $41.35_{-}^{+} 0.02$ \\
\hline & $X M M-N e w t o n$ & 0505140401 & 2007-07-02 & 20 & 78 & 49947 & $U V W 1$ & $42.08_{-}^{+} 0.01$ \\
\hline & XMM-Newton & 0505140501 & 2007-07-04 & 20 & 46 & 29528 & $U V W 1$ & $42.24_{-}^{+} 0.01$ \\
\hline & & & & & & & $U V M 2$ & $42.04_{-}^{+} 0.01$ \\
\hline & XMM-Newton & 0692840201 & $2012-07-25$ & 20 & 106 & 115167 & $U V W 1$ & $42.01_{-}^{+} 0.01$ \\
\hline & XMM-Newton & 0692840301 & $2012-12-24$ & 20 & 95 & 276846 & $U V W 1$ & $41.94_{-}^{+} 0.01$ \\
\hline & XMM-Newton & 0692840401 & 2013-01-23 & 20 & 89 & 366687 & $U V W 1$ & $41.78_{-}^{+} 0.01$ \\
\hline & XMM-Newton & 0692840501 & 2013-02-12 & 20 & 102 & 172790 & $U V W 1$ & $41.77_{-}^{+} 0.01$ \\
\hline \multirow[t]{4}{*}{ NGC 2617} & XMM-Newton & 0701981601 & 2013-04-27 & 25 & 39 & 574119 & $U V W 2$ & $42.95_{-}^{+} 0.02$ \\
\hline & & & & & & & $U V M 2$ & $43.02_{-}^{+} 0.01$ \\
\hline & & & & & & & $U V W 1$ & $43.05_{-}^{+} 0.01$ \\
\hline & XMM-Newton & 0701981901 & 2013-05-24 & 25 & 14 & 496297 & $U V W 1$ & $43.24_{-}^{+} 0.01$ \\
\hline
\end{tabular}

Notes. (Column 1) name, (Col. 2) instrument, (Col. 3) obsID, (Col. 4) date, (Col. 5) aperture radius for the nuclear extraction, (Col. 6) net exposure time, (Col. 7) number of counts in the $0.5-10 \mathrm{keV}$ band, (Cols. 8 and 9) UV luminosity from the optical monitor and filter. The ${ }^{c}$ represents data from different instruments that were compared as explained in Sect. 4.2. 
Table A.1. continued.

\begin{tabular}{|c|c|c|c|c|c|c|c|c|}
\hline Name & Instrument & ObsID & Date & $\begin{array}{l}R \\
\left({ }^{\prime \prime}\right) \\
(5)\end{array}$ & $\begin{array}{c}\text { Net exptime } \\
\text { (ks) } \\
\text { (6) }\end{array}$ & Counts & OM Filter & $\begin{array}{c}\log \left(L_{\mathrm{UV}}\right) \\
(\mathrm{erg} / \mathrm{s}) \\
(9)\end{array}$ \\
\hline \multirow[t]{4}{*}{ MARK 1218} & XMM-Newton & 0302260201 & 20050409 & 20 & 6 & 3652 & $U V W 2$ & $41.98_{-}^{+} 0.25$ \\
\hline & & & & & & & $U V M 2$ & $42.12_{-}^{+} 0.09$ \\
\hline & & & & & & & $U V W 1$ & $42.43_{-}^{+} 0.03$ \\
\hline & XMM-Newton & 0302260401 & 20051008 & 20 & 3 & 935 & UVM2 & $42.06_{-}^{+} 0.10$ \\
\hline \multirow[t]{8}{*}{ NGC 2992} & XMM-Newton & 0654910501 & $2010-05-26$ & 25 & 35 & 85124 & UVM2 & $41.14_{-}^{+} 0.08$ \\
\hline & $X M M-N e w t o n$ & 0654910601 & 2010-06-05 & 25 & 33 & 35540 & $U V M 2$ & $41.28_{-}^{+} 0.05$ \\
\hline & XMM-Newton & 0654910701 & 2010-11-08 & 25 & 37 & 40296 & & Not detected \\
\hline & XMM-Newton & 0654910901 & 2010-11-28 & 25 & 33 & 20874 & & Not detected \\
\hline & XMM-Newton & 0654911001 & 2010-12-08 & 25 & 38 & 44100 & $U V W 2$ & $40.70_{-}^{+} 0.33$ \\
\hline & & & & & & & $U V M 2$ & $41.24_{-}^{+} 0.03$ \\
\hline & & & & & & & $U V W 1$ & $41.87_{-}^{+} 0.01$ \\
\hline & XMM-Newton & 0701780101 & 2013-05-11 & 25 & 9 & 26676 & UVM2 & $41.09_{-}^{+} 0.06$ \\
\hline \multirow[t]{2}{*}{ POX 52} & Chandra $^{c}$ & 5736 & 2006-04-18 & 2 & 22 & 4979 & - & \\
\hline & XMM-Newton ${ }^{c}$ & 0302420101 & 2005-07-08 & 20 & 69 & 4869 & UVM2 & $42.21_{-}^{+} 0.03$ \\
\hline \multirow[t]{2}{*}{ NGC 4138} & Chandra $^{c}$ & 3994 & 2003-03-16 & 2 & 6 & 889 & - & \\
\hline & XMM-Newton ${ }^{c}$ & 0112551201 & 2001-11-26 & 25 & 8 & 4271 & - & \\
\hline \multirow[t]{5}{*}{ NGC 4395} & Chandra & 5302 & 2004-04-11 & 2 & 28 & 2779 & - & \\
\hline & Chandra ${ }^{c}$ & 5301 & 2004-04-10 & 2 & 27 & 3105 & - & \\
\hline & $X M M-$ Newton $^{c}$ & 0112521901 & 2002-05-31 & 25 & 8 & 5209 & $U V W 1$ & $40.09_{-}^{+} 0.02$ \\
\hline & XMM-Newton & 0744010101 & $2014-12-28$ & 25 & 36 & 16941 & $U V W 1$ & $39.99_{-}^{+} 0.01$ \\
\hline & XMM-Newton & 0744010201 & 2014-12-31 & 25 & 14 & 8819 & $U V W 1$ & $40.07_{-}^{+} 0.01$ \\
\hline \multirow[t]{2}{*}{ NGC 4565} & XMM-Newton ${ }^{c}$ & 0112550301 & 2001-07-01 & 20 & 9 & 1209 & - & \\
\hline & Chandra $^{c}$ & 3950 & 2003-02-08 & 2 & 59 & 2158 & - & \\
\hline \multirow[t]{10}{*}{ MARK 883} & $X M M-N e w t o n$ & 0302260101 & 2006-08-13 & 25 & 8 & 5311 & $U V W 2$ & $42.99_{-}^{+} 0.08$ \\
\hline & & & & & & & $U V M 2$ & $42.92_{-}^{+} 0.04$ \\
\hline & & & & & & & $U V W 1$ & $43.01_{-}^{+} 0.02$ \\
\hline & XMM-Newton & 0302260701 & 2006-08-15 & 25 & 10 & 7307 & $U V W 2$ & $42.85_{-}^{+} 0.06$ \\
\hline & & & & & & & $U V M 2$ & $42.91_{-}^{+} 0.04$ \\
\hline & & & & & & & $U V W 1$ & $43.02_{-}^{+} 0.02$ \\
\hline & XMM-Newton & 0302261001 & 2006-08-21 & 25 & 8 & 7000 & $U V W 2$ & $42.75_{-}^{+} 0.06$ \\
\hline & & & & & & & $U V M 2$ & $42.94_{-}^{+} 0.04$ \\
\hline & & & & & & & $U V W 1$ & $43.03_{-}^{+} 0.02$ \\
\hline & XMM-Newton & 0652550201 & 2010-08-20 & 25 & 26 & 12409 & $U V W 1$ & $43.13_{-}^{+} 0.01$ \\
\hline \multirow[t]{3}{*}{ IRAS 2005-1117 } & $X M M-N e w t o n$ & 0044350201 & 2002-04-20 & 20 & 4 & 4535 & $U V W 2$ & $42.65_{-}^{+} 0.09$ \\
\hline & & & & & & & $U V W 1$ & $43.03_{-}^{+} 0.01$ \\
\hline & XMM-Newton & 0044350501 & 2002-10-21 & 20 & 10 & 7020 & $U V W 1$ & $43.01_{-}^{+} 0.02$ \\
\hline
\end{tabular}


Table A.2. Final compilation of the best-fit models for the sample.

\begin{tabular}{|c|c|c|c|c|c|c|c|c|c|}
\hline Analysis & ObsID & Model & $N_{\mathrm{H} 1}$ & $N_{\mathrm{H} 2}$ & $\begin{array}{c}k T \\
\mathrm{keV} \\
(6)\end{array}$ & (7) & $\begin{array}{c}\text { Norm }_{1} \\
\left(10^{-4}\right) \\
(8)\end{array}$ & $\begin{array}{c}\text { Norm }_{2} \\
\left(10^{-4}\right) \\
(9)\end{array}$ & $\begin{array}{c}\chi^{2} / \text { d.o.f. } \\
F \text {-test } \\
(10)\end{array}$ \\
\hline \multicolumn{10}{|c|}{ ESO 540-G01 } \\
\hline Ind & 2192 & MEPL & $0_{0}^{0.45}$ & $0.04_{0}^{0.14}$ & $0.36_{0.20}^{0.52}$ & $1.89_{1.69}^{2.15}$ & $0.17_{0.08}^{2.73}$ & $0.69_{0.55}^{0.84}$ & $46.20 / 42$ \\
\hline Ind & 0044350101 & MEPL & - & $0.75_{0.63}^{1.15}$ & $0.65_{0.33}^{0.80}$ & $1.82_{1.74}^{1.90}$ & $0.90_{0.81}^{0.99}$ & $0.08_{0.01}^{6.03}$ & $87.51 / 92$ \\
\hline SMF (+ring) & $\begin{array}{c}0044350101 \\
2129\end{array}$ & MEPL & - & - & $0.38_{0.31}^{0.55}$ & $1.68_{1.61}^{1.76}$ & $\begin{array}{l}0.15_{0.10}^{0.20} \\
0.57_{0.53}^{0.62} \\
\end{array}$ & $0.87_{0.82}^{0.93}$ & $137.15 / 142$ \\
\hline \multicolumn{10}{|c|}{ ESO 195-IG21 } \\
\hline Ind & 13898 & MEPL & $5.34_{3.07}^{8.25}$ & $10.43_{5.98}^{19.62}$ & $0.27_{0.14}^{1.11}$ & $2.42_{1.17}^{0}$ & $252.77_{2.03}^{330.35}$ & $9.58_{5.05}^{135.83}$ & $15.09 / 16$ \\
\hline Ind & 0554500201 & MEPL & $0.79_{0.68}^{0.93}$ & $3.39_{3.11}^{3.69}$ & $0.14_{0.11}^{0.17}$ & $1.32_{1.22}^{1.43}$ & $46.69_{11.98}^{308.08}$ & $4.87_{4.10}^{5.82}$ & $274.21 / 262$ \\
\hline SMF (+ring) & $\begin{array}{c}0554500201 \\
2012\end{array}$ & MEPL & $0.27_{0.00}^{2.11}$ & $3.81_{3.15}^{4.65}$ & $2.67_{0.38}^{4.85}$ & $1.35_{1.24}^{1.48}$ & $\begin{array}{c}0.47_{0.13}^{2.23} \\
0.04_{0.01}^{0.12} \\
\end{array}$ & $\begin{array}{r}5.15_{4.21}^{6.43} \\
0.08_{0.03}^{0.14} \\
\end{array}$ & $270.70 / 255$ \\
\hline \multicolumn{10}{|c|}{ ESO 113-G10 } \\
\hline Ind & 0103861601 & ME2PL & - & $1.57_{0.99}^{2.82}$ & 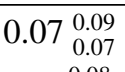 & $\begin{array}{l}3.27_{3.18}^{3.40} \\
\end{array}$ & $6.07_{6.07}^{7.37}$ & $6.12_{2.72}^{12.09}$ & $277.61 / 236$ \\
\hline Ind & 0301890101 & ME2PL & - & $4.02_{3.73}^{4.35}$ & $0.07_{0.07}^{0.08}$ & $2.63_{2.60}^{2.66}$ & $14.76_{14.76}^{14.96}$ & $19.33_{17.97}^{20.76}$ & $2080.8 / 1088$ \\
\hline SMF1 & $\begin{array}{l}0103861601 \\
0301890101\end{array}$ & ME2PL & - & $3.62_{3.41}^{3.87}$ & $0.08_{0.07}^{0.09}$ & $2.67_{2.65}^{2.70}$ & $\begin{array}{c}8.95_{8.73}^{9.17} \\
14.81_{14.70}^{14.91} \\
\end{array}$ & $20.48_{19.13}^{21.91}$ & $2281.77 / 1302$ \\
\hline \multicolumn{10}{|c|}{ NGC 526A } \\
\hline Ind & 0109130201 & $2 \mathrm{PL}$ & $0.01_{0}^{0.06}$ & $1.14_{1.08}^{1.24}$ & - & $1.38_{1.34}^{1.43}$ & $1.08_{0.97}^{1.58}$ & $25.31_{23.72}^{27.13}$ & $706.22 / 732$ \\
\hline Ind & 0150940101 & $2 \mathrm{PL}$ & $0.03_{0}^{0.05}$ & $1.17_{1.14}^{1.21}$ & - & $1.48_{1.46}^{1.50}$ & $1.25_{1.04}^{1.49}$ & $39.74_{38.67}^{40.86}$ & $1609.78 / 1538$ \\
\hline Ind & 0721730301 & $2 \mathrm{PL}$ & $0.03_{0.01}^{0.05}$ & $1.25_{1.22}^{1.28}$ & - & $1.49_{1.47}^{1.50}$ & $1.37_{1.19}^{1.58}$ & $46.44_{45.37}^{47.53}$ & $1718.2 / 1638$ \\
\hline Ind & 0721730401 & $2 \mathrm{PL}$ & $0.06_{0.03}^{0.09}$ & $1.23_{1.20}^{1.27}$ & - & $1.50_{1.48}^{1.51}$ & $1.71_{1.44}^{2.02}$ & $55.94_{54.64}^{57.30}$ & $1747.04 / 1614$ \\
\hline Ind & 342 & $2 \mathrm{PL}$ & $0.73_{0.69}^{1.04}$ & $120.02_{64.85}^{185.04}$ & - & $0.97_{0.84}^{1.25}$ & $2.40_{1.99}^{2.78}$ & $16.32_{4.02}^{25.17}$ & $61.32 / 45$ \\
\hline SMF1 & 0109130201 & $2 \mathrm{PL}$ & $0.03_{0.02}^{0.04}$ & $1.21_{1.20}^{1.23}$ & - & $1.48_{1.47}^{1.49}$ & $1.36_{1.24}^{1.48}$ & $28.49_{27.97}^{29.02}$ & $5863.99 / 5543$ \\
\hline & 0150940101 & & & & & & & $40.16_{39.59}^{40.74}$ & \\
\hline & 0721730301 & & & & & & & $45.72_{45.07}^{46.38}$ & \\
\hline & 0721730401 & & & & & & & $55.12_{54.33}^{55.93}$ & \\
\hline \multicolumn{10}{|c|}{ MARK 609} \\
\hline Ind & 0103861001 & $2 \mathrm{PL}$ & - & $10.43_{5.28}^{149.71}$ & - & $1.96_{1.89}^{2.03}$ & $3.78_{3.67}^{3.89}$ & $2.21_{1.18}^{46.21}$ & $183.79 / 173$ \\
\hline Ind & 0402110201 & $2 \mathrm{PL}$ & - & $12.62_{7.69}^{24.95}$ & - & $1.81_{1.78}^{1.89}$ & $2.90_{2.84}^{2.96}$ & $2.45_{1.80}^{3.31}$ & $359.17 / 319$ \\
\hline SMF1 & 0103861001 & $2 \mathrm{PL}$ & - & $11.41_{7.16}^{17.41}$ & - & $1.89_{1.85}^{1.93}$ & $3.71_{3.61}^{3.81}$ & $2.58_{1.95}^{3.16}$ & $566.07 / 499$ \\
\hline & 0402110201 & & & & & & $2.91_{2.85}^{2.97}$ & & \\
\hline \multicolumn{10}{|c|}{ NGC 1365} \\
\hline Ind & 6869 & 2PL & $0_{0}^{0.05}$ & $35.98_{33.47}^{38.60}$ & - & $1.53_{1.35}^{1.76}$ & $0.46_{0.43}^{0.54}$ & $38.56_{27.19}^{61.78}$ & $126.46 / 89$ \\
\hline Ind & 0151370101 & ME2PL & - & $40.07_{37.58}^{42.76}$ & $0.64_{0.61}^{0.66}$ & $1.90_{1.83}^{1.96}$ & $1.60_{1.59}^{1.76}$ & $56.68_{48.52}^{66.66}$ & $421.94 / 359$ \\
\hline Ind & 0151370201 & ME2PL & - & $13.30_{8.30}^{22.30}$ & $0.73_{0.65}^{0.82}$ & $2.02_{1.83}^{2.22}$ & $1.57_{1.57}^{2.01}$ & $9.21_{5.56}^{14.60}$ & $70.21 / 49$ \\
\hline Ind & 0151370701 & ME2PL & $0_{0}^{0.04}$ & $32.58_{30.26}^{34.62}$ & $0.68_{0.65}^{0.71}$ & $2.12_{2.04}^{2.38}$ & $1.71_{1.59}^{2.02}$ & $97.42_{97.41}^{188.31}$ & $335.83 / 232$ \\
\hline Ind & 0205590301 & ME2PL & $0.06_{0.05}^{0.06}$ & $11.95_{11.81}^{12.12}$ & $0.62_{0.60}^{0.63}$ & $2.18_{2.17}^{2.22}$ & $2.65_{2.65}^{2.80}$ & $130.56_{125.17}^{144.97}$ & $2751.27 / 1504$ \\
\hline
\end{tabular}

Notes. Including the individual best-fit model for each observation, and the simultaneous best-fit model (i.e., SMF0, SMF1, SMF2, or SMF3) with the varying parameters (except for SMF0). Note that when the column density is compatible with the Galactic absorption, a "--" is placed. 
Table A.2. continued.

\begin{tabular}{|c|c|c|c|c|c|c|c|c|c|}
\hline Analysis & ObsID & Model & $N_{\mathrm{H} 1}$ & $N_{\mathrm{H} 2}$ & $\begin{array}{c}k T \\
\mathrm{keV} \\
(6)\end{array}$ & (7) & $\begin{array}{c}\text { Norm }_{1} \\
\left(10^{-4}\right) \\
(8)\end{array}$ & $\begin{array}{c}\text { Norm }_{2} \\
\left(10^{-4}\right) \\
(9)\end{array}$ & $\begin{array}{c}\chi^{2} / \text { d.o.f. } \\
F \text {-test } \\
(10)\end{array}$ \\
\hline Ind & 0205590401 & ME2PL & - & $25.86_{24.72}^{26.41}$ & $0.62_{0.61}^{0.64}$ & $2.03_{1.98}^{2.08}$ & $1.65_{1.65}^{1.77}$ & $80.55_{72.16}^{88.22}$ & $1181.26 / 778$ \\
\hline Ind & 0505140201 & ME2PL & - & $52.73_{46.76}^{59.11}$ & $0.65_{0.64}^{0.66}$ & $2.20_{2.16}^{2.25}$ & $1.53_{1.52}^{1.62}$ & $25.72_{21.58}^{30.56}$ & $632.85 / 444$ \\
\hline Ind & 0505140401 & ME2PL & - & $64.17_{60.03}^{65.92}$ & $0.64_{0.63}^{0.65}$ & $2.15_{2.13}^{2.20}$ & $1.57_{1.57}^{1.63}$ & $45.38_{41.01}^{50.70}$ & $977.71 / 681$ \\
\hline Ind & 0505140501 & ME2PL & - & $60.11_{54.24}^{66.62}$ & $0.63_{0.62}^{0.65}$ & $2.14_{2.10}^{2.17}$ & $1.57_{1.57}^{1.65}$ & $35.33_{30.12}^{41.61}$ & $700.17 / 499$ \\
\hline Ind & 0692840201 & ME2PL & - & $26.07_{25.71}^{26.43}$ & $0.65_{0.64}^{0.66}$ & $2.12_{2.10}^{2.15}$ & $1.69_{1.66}^{1.72}$ & $104.65_{104.65}^{115.25}$ & $2186.8 / 1529$ \\
\hline Ind & 0692840301 & ME2PL & - & $3.66_{3.60}^{3.72}$ & $0.61_{0.58}^{0.64}$ & $1.40_{1.39}^{1.42}$ & $4.91_{4.91}^{5.06}$ & $33.35_{32.41}^{34.32}$ & $5588.28 / 1703$ \\
\hline Ind & 0692840401 & ME2PL & $0.09_{0.09}^{0.09}$ & $1.99_{1.97}^{2.02}$ & $67.07_{0}^{0}$ & $1.65_{1.64}^{1.67}$ & $10.95_{10.95}^{11.28}$ & $46.00_{45.13}^{47.33}$ & $4283.19 / 1697$ \\
\hline Ind & 0692840501 & ME2PL & - & $12.30_{12.14}^{12.43}$ & $0.63_{0.62}^{0.64}$ & $1.74_{1.73}^{1.76}$ & $2.73_{2.73}^{2.80}$ & $61.81_{59.72}^{63.43}$ & $2817.83 / 1687$ \\
\hline \multirow[t]{6}{*}{ SMF3 } & 0151370101 & ME2PL & - & $39.40_{37.04}^{41.93}$ & $0.62_{0.61}^{0.62}$ & $1.63_{1.62}^{1.64}$ & $1.63_{1.58}^{1.69}$ & $58.68_{54.30}^{63.43}$ & $10738.3 / 6122$ \\
\hline & 0151370701 & & & $31.17_{29.45}^{33.02}$ & & & $1.74_{1.66}^{1.83}$ & $\begin{array}{r}73.99 \\
69.02\end{array}$ & \\
\hline & 0205590301 & & & $10.44_{10.28}^{10.59}$ & & & $2.07_{2.03}^{2.11}$ & $79.99_{77.90}^{82.13}$ & \\
\hline & 0205590401 & & & $25.36_{24.67}^{26.07}$ & & & $1.79_{1.75}^{1.80}$ & $65.26_{62.82}^{67.77}$ & \\
\hline & 0692840201 & & & $25.18_{24.84}^{25.52}$ & & & $1.77_{1.75}^{1.80}$ & $74.12_{72.03}^{76.25}$ & \\
\hline & 0692840501 & & & $\begin{array}{r}12.77_{12.62}^{12.93} \\
\end{array}$ & & & $2.52_{2.49}^{2.55}$ & $87.64_{85.42}^{89.91}$ & \\
\hline \multirow[t]{2}{*}{ SMF2 (+ring) } & 0205590401 & ME2PL & - & $27.74_{25.62}^{28.95}$ & $<0.69$ & $2.26_{2.18}^{2.51}$ & $0.31_{0.24}^{0.37}$ & $140.24_{99.61}^{185.74}$ & $1388.72 / 873$ \\
\hline & 6869 & & & $44.27_{40.82}^{45.58}$ & & & & $199.22_{158.50}^{259.85}$ & \\
\hline \multicolumn{10}{|c|}{ NGC 2617} \\
\hline Ind & 0701981601 & $2 \mathrm{PL}$ & - & $11.93_{10.44}^{13.65}$ & - & $2.01_{2.00}^{2.02}$ & $71.01_{70.80}^{71.21}$ & $39.95_{37.52}^{42.55}$ & $1903.22 / 1564$ \\
\hline Ind & 0701981901 & $2 \mathrm{PL}$ & - & $10.07_{8.61}^{11.77}$ & - & $2.15_{2.14}^{2.16}$ & $0.02_{0.02}^{0.02}$ & $0.01_{0.01}^{0.01}$ & $1861.39 / 1469$ \\
\hline \multirow[t]{2}{*}{ SMF2 } & 0701981601 & $2 \mathrm{PL}$ & - & $6.88_{6.25}^{7.54}$ & - & $2.08_{2.07}^{2.09}$ & $70.05_{69.81}^{70.28}$ & $49.41_{47.49}^{51.41}$ & $4431.52 / 3039$ \\
\hline & 0701981901 & & & $9.80_{8.75}^{10.98}$ & & & $170.94_{170.46}^{171.43}$ & & \\
\hline \multicolumn{10}{|c|}{ MARK 1218} \\
\hline Ind & 0302260201 & PL & $0.09_{0.06}^{0.12}$ & - & - & $1.12_{1.06}^{1.20}$ & - & - & $187.44 / 146$ \\
\hline Ind & 0302260401 & PL & - & - & - & $0.81_{0.74}^{0.94}$ & - & - & $27.40 / 30$ \\
\hline \multirow[t]{2}{*}{ SMF1 } & 0302260201 & PL & $0.06_{0.03}^{0.09}$ & - & - & $1.05_{1.00}^{1.12}$ & $2.49_{2.31}^{2.72}$ & - & $231.136 / 181$ \\
\hline & & & & & & & $0.91_{0.84}^{1.00}$ & & \\
\hline \multicolumn{10}{|c|}{ NGC 2992} \\
\hline Ind & 0654910501 & $2 \mathrm{PL}$ & $0.16_{0.12}^{0.19}$ & $0.93_{0.85}^{1.02}$ & - & $1.50_{1.48}^{1.53}$ & $4.78_{3.74}^{5.88}$ & $20.42_{19.45}^{21.35}$ & $1460.82 / 1412$ \\
\hline Ind & 0654910601 & $2 \mathrm{PL}$ & $0.12_{0.10}^{0.14}$ & $1.18_{0.92}^{1.47}$ & - & $1.38_{1.32}^{1.44}$ & $3.93_{3.42}^{4.35}$ & $4.05_{3.44}^{4.70}$ & $1078.77 / 947$ \\
\hline Ind & 0654910701 & $2 \mathrm{PL}$ & $0.11_{0.08}^{0.14}$ & $0.99_{0.77}^{1.24}$ & - & $1.37_{1.32}^{1.42}$ & $3.72_{3.09}^{4.21}$ & $4.22_{3.66}^{4.78}$ & $1012.9 / 1016$ \\
\hline Ind & 0654910901 & $2 \mathrm{PL}$ & $0.05_{0.03}^{0.07}$ & $8.13_{2.36}^{57.00}$ & - & $1.22_{1.10}^{1.34}$ & $2.65_{2.48}^{2.83}$ & $0.54_{0.48}^{1.21}$ & 676.2/664 \\
\hline Ind & 0654911001 & $2 \mathrm{PL}$ & $0.08_{0.05}^{0.11}$ & $0.85_{0.69}^{1.03}$ & - & $1.37_{1.33}^{1.42}$ & $2.99_{2.29}^{3.59}$ & $5.79_{5.24}^{6.33}$ & $1106.19 / 1078$ \\
\hline Ind & 0701780101 & $2 \mathrm{PL}$ & $0.09_{0}^{0.17}$ & $0.81_{0.70}^{0.95}$ & - & $1.50_{1.46}^{1.55}$ & $3.36_{1.68}^{5.75}$ & $27.24_{25.32}^{28.99}$ & $818.68 / 821$ \\
\hline \multirow[t]{6}{*}{ SMF2 } & 0654910501 & $2 \mathrm{PL}$ & $0.09_{0.08}^{0.09}$ & $0.76_{0.74}^{0.78}$ & - & $1.43_{1.41}^{1.44}$ & $2.99_{2.90}^{3.07}$ & $19.52_{19.06}^{20.00}$ & $6291.22 / 5968$ \\
\hline & 0654910601 & & & $0.95_{0.89}^{1.01}$ & & & & $5.51_{5.30}^{5.73}$ & \\
\hline & 0654910701 & & & $0.88_{0.83}^{0.93}$ & & & & $5.53_{5.33}^{5.74}$ & \\
\hline & 0654910901 & & & $7.44_{5.36}^{10.37}$ & & & & $1.76_{1.53}^{2.02}$ & \\
\hline & 0654911001 & & & $0.93_{0.88}^{0.98}$ & & & & $6.56_{6.34}^{6.79}$ & \\
\hline & 0701780101 & & & $0.72_{0.69}^{0.74}$ & & & & $24.66_{24.02}^{25.32}$ & \\
\hline
\end{tabular}


Table A.2. continued.

\begin{tabular}{|c|c|c|c|c|c|c|c|c|c|}
\hline Analysis & ObsID & Model & $N_{\mathrm{H} 1}$ & $N_{\mathrm{H} 2}$ & $\begin{array}{c}k T \\
\mathrm{keV} \\
(6)\end{array}$ & (7) & $\begin{array}{c}\text { Norm }_{1} \\
\left(10^{-4}\right) \\
(8)\end{array}$ & $\begin{array}{c}\text { Norm }_{2} \\
\left(10^{-4}\right) \\
(9)\end{array}$ & $\begin{array}{c}\chi^{2} / \text { d.o.f. } \\
F \text {-test } \\
(10)\end{array}$ \\
\hline Ind & 5736 & ME2PL & - & $3.23_{2.36}^{4.44}$ & $0.22_{0.20}^{0.25}$ & $2.62_{2.44}^{2.78}$ & $2.32_{2.32}^{2.69}$ & $4.25_{2.65}^{6.13}$ & $122.38 / 117$ \\
\hline Ind & 0302420101 & ME2PL & - & $\begin{array}{r}6.597 .39 \\
5.79 \\
\end{array}$ & $0.16_{0.14}^{0.18}$ & $1.79_{1.60}^{1.98}$ & $0.11_{0.11}^{0.13}$ & $1.33_{0.92}^{1.89}$ & $244.71 / 174$ \\
\hline SMF (+ring) & $\begin{array}{c}0302420101 \\
5736\end{array}$ & ME2PL & $\begin{array}{c}8.38_{7.36}^{10.43} \\
-\end{array}$ & $\begin{array}{l}2.43_{1.30}^{4.28} \\
4.32_{3.51}^{5.41}\end{array}$ & $0.15_{0.11}^{0.17}$ & $2.30_{2.26}^{2.34}$ & $2.76_{2.05}^{2.98}$ & $0.30_{0.10}^{1.00}$ & $378.76 / 290$ \\
\hline \multicolumn{10}{|c|}{ NGC 4138} \\
\hline Ind & 3994 & PL & $\begin{array}{l}7.92_{6.62}^{9.95} \\
\end{array}$ & - & - & $1.36_{1.01}^{1.93}$ & - & - & $34.66 / 35$ \\
\hline Ind & 0112551201 & PL(2-10 keV) & $7.89_{7.00}^{8.82}$ & - & - & $1.44_{1.26}^{1.62}$ & & $0.14_{0.10}^{0.19}$ & $153.80 / 162$ \\
\hline SMF (+ring) & $\begin{array}{c}0112551201 \\
2003\end{array}$ & $\mathrm{PL}(2-10 \mathrm{keV})$ & $6.76_{4.22}^{9.55}$ & - & - & $1.20_{0.61}^{1.83}$ & $\begin{array}{c}0.19_{0.01}^{0.86} \\
8.91_{3.16}^{26.70} \\
\end{array}$ & - & $186.04 / 190$ \\
\hline \multicolumn{10}{|c|}{ NGC 4395} \\
\hline Ind & 5302 & ME2PL & - & $4.00_{3.52}^{4.52}$ & $0.09_{0.05}^{0.17}$ & $1.13_{0.94}^{1.33}$ & $0.07_{0.07}^{0.12}$ & $3.99_{2.90}^{5.52}$ & $99.62 / 108$ \\
\hline Ind & 5301 & ME2PL & - & $3.40_{3.04}^{3.76}$ & $0.08_{0.03}^{0.22}$ & $1.52_{1.30}^{1.83}$ & $0.14_{0.11}^{0.16}$ & $5.98_{5.98}^{10.70}$ & $183.22 / 121$ \\
\hline SMF1 & $\begin{array}{l}5302 \\
5301\end{array}$ & ME2PL & - & $\begin{array}{r}4.34_{3.99}^{4.69} \\
3.01_{2.74}^{3.29} \\
\end{array}$ & $0.09_{0.05}^{0.16}$ & $1.37_{1.24}^{1.50}$ & $0.11_{0.09}^{0.13}$ & $5.50_{4.46}^{6.81}$ & $265.8 / 238$ \\
\hline Ind & 0112521901 & ME2PL & $5.71_{5.11}^{6.45}$ & $0.04_{0}^{0.23}$ & $0.16_{0.09}^{0.21}$ & $1.29_{1.12}^{1.52}$ & $6.75_{6.75}^{11.53}$ & $0.35_{0.29}^{0.49}$ & $261.70 / 220$ \\
\hline Ind & 0744010101 & ME2PL & - & $8.14_{7.67}^{8.63}$ & $0.19_{0.18}^{0.20}$ & $1.27_{1.18}^{1.35}$ & $0.30_{0.29}^{0.34}$ & $8.06_{6.84}^{9.51}$ & $601.99 / 590$ \\
\hline Ind & 0744010201 & ME2PL & - & $4.78_{4.56}^{5.20}$ & $0.18_{0.14}^{0.21}$ & $1.16_{1.05}^{1.26}$ & $0.36_{0.35}^{0.47}$ & $7.49_{6.14}^{8.93}$ & $369.14 / 344$ \\
\hline \multirow[t]{3}{*}{ SMF2 } & 0112521901 & ME2PL & - & $5.81_{5.43}^{6.21}$ & $0.19_{0.18}^{0.20}$ & $1.20_{1.14}^{1.26}$ & $0.33_{0.31}^{0.35}$ & $9.40_{8.37}^{10.55}$ & $1217.45 / 1170$ \\
\hline & 0744010101 & & & $7.94_{7.56}^{8.34}$ & & & & $70.83_{63.06}^{79.57}$ & \\
\hline & 0744010201 & & & $\begin{array}{l}4.66_{4.38}^{4.96} \\
\end{array}$ & & & & $80.75_{72.23}^{90.29}$ & \\
\hline SMF1 (+ring) & $\begin{array}{c}0112521901 \\
5301\end{array}$ & ME2PL & $2.87_{2.55}^{3.20}$ & $13.58_{11.38}^{16.19}$ & $0.25_{0.24}^{0.27}$ & $1.42_{1.26}^{1.64}$ & $5.31_{4.23}^{6.82}$ & $\begin{array}{c}10.17_{6.54}^{17.25} \\
0.76_{0.01}^{3.00} \\
\end{array}$ & $460.32 / 350$ \\
\hline \multicolumn{10}{|c|}{ NGC 4565} \\
\hline Ind & 0112550301 & PL & $0.11_{0.07}^{0.16}$ & - & - & $1.80_{1.67}^{1.99}$ & - & - & $46.52 / 46$ \\
\hline Ind & 3950 & PL & $0.25_{0.22}^{0.28}$ & - & - & $1.96_{1.85}^{2.05}$ & - & - & $71.19 / 77$ \\
\hline SMF (+ring) & $\begin{array}{c}0112550301 \\
3950\end{array}$ & PL & $\begin{array}{l}0.13_{0.10}^{0.17} \\
0.25_{0.22}^{0.29} \\
\end{array}$ & - & - & $1.94_{1.85}^{2.05}$ & $0.78_{0.71}^{0.86}$ & - & $121.88 / 128$ \\
\hline \multicolumn{10}{|c|}{ MARK 883} \\
\hline Ind & 0302260101 & PL & $0.04_{0.02}^{0.06}$ & - & - & $1.61_{1.54}^{1.68}$ & - & - & $206.37 / 191$ \\
\hline Ind & 0302260701 & PL & $0.04_{0.02}^{0.06}$ & - & - & $1.64_{1.59}^{1.71}$ & - & - & $269.11 / 249$ \\
\hline Ind & 0302261001 & PL & $0.07_{0.05}^{0.08}$ & - & - & $1.75_{1.69}^{1.81}$ & - & - & $221.43 / 243$ \\
\hline Ind & 0652550201 & PL & $0.04_{0.03}^{0.05}$ & - & - & $1.66_{1.62}^{1.71}$ & - & - & $438.40 / 407$ \\
\hline SMF1 & 0302260101 & $\mathrm{PL}$ & $0.05_{0.04}^{0.05}$ & - & - & $1.67_{1.64}^{1.70}$ & & $3.67_{3.54}^{3.80}$ & $1151.81 / 1105$ \\
\hline & 0302260701 & & & & & & & $3.64_{3.52}^{3.76}$ & \\
\hline & 0302261001 & & & & & & & $4.62_{4.47}^{4.77}$ & \\
\hline & 0652550201 & & & & & & & $2.66_{2.59}^{2.74}$ & \\
\hline \multicolumn{10}{|c|}{ IRAS 20051-1117 } \\
\hline Ind & 0044350201 & PL & - & - & - & $1.89_{1.85}^{1.94}$ & - & - & $171.36 / 160$ \\
\hline Ind & 0044350501 & PL & - & - & - & $1.93_{1.89}^{1.96}$ & - & - & $246.53 / 245$ \\
\hline SMF1 & 0044350201 & PL & - & - & - & $1.91_{1.88}^{1.94}$ & - & $5.88_{5.73}^{6.04}$ & $425.12 / 410$ \\
\hline
\end{tabular}


Table A.2. continued.

\begin{tabular}{|c|c|c|c|c|c|c|c|c|c|}
\hline Analysis & ObsID & Model & $N_{\mathrm{H} 1}$ & $N_{\mathrm{H} 2}$ & $\begin{array}{c}k T \\
\mathrm{keV}\end{array}$ & $\Gamma$ & $\begin{array}{l}\text { Norm }_{1} \\
\left(10^{-4}\right)\end{array}$ & $\begin{array}{c}\text { Norm }_{2} \\
\left(10^{-4}\right)\end{array}$ & $\begin{array}{c}\chi^{2} / \text { d.o.f } \\
F \text {-test }\end{array}$ \\
\hline (1) & (2) & (3) & (4) & (5) & (6) & (7) & (8) & (9) & (10) \\
\hline & 0044350501 & & & & & & & $4.17_{4.08}^{4.26}$ & \\
\hline
\end{tabular}

Table A.3. X-ray luminosities.

\begin{tabular}{|c|c|c|c|c|c|c|}
\hline \multirow{3}{*}{$\begin{array}{l}\text { Name } \\
\text { (1) }\end{array}$} & \multirow{3}{*}{$\begin{array}{c}\text { Satellite } \\
\text { (2) }\end{array}$} & \multirow{3}{*}{$\begin{array}{c}\text { ObsID } \\
(3)\end{array}$} & \multicolumn{2}{|l|}{ Individual } & \multicolumn{2}{|c|}{ Simultaneous } \\
\hline & & & $\log (L(0.5-2 \mathrm{keV}))$ & $\log (L(2-10 \mathrm{keV}))$ & $\log (L(0.5-2 \mathrm{keV}))$ & $\log (L(2-10 \mathrm{keV}))$ \\
\hline & & & (4) & (5) & (6) & (7) \\
\hline \multirow[t]{3}{*}{ ESO 540-G01 } & XMM-Newton & 0044350101 & $43.38_{43.36}^{43.40}$ & $41.83 \begin{array}{c}41.86 \\
41.79\end{array}$ & $\begin{array}{l}41.63_{41.62}^{41.65} \\
\end{array}$ & $41.81_{41.77}^{41.84}$ \\
\hline & Chandra $\left(3^{\prime \prime}\right)$ & 2192 & $41.49_{41.42}^{41.54}$ & $41.55_{41.02}^{44.12}$ & $41.41_{41.39}^{41.43}$ & $41.60_{41.57}^{41.64}$ \\
\hline & Chandra $\left(25^{\prime \prime}\right)$ & 2192 & $41.80_{41.73}^{41.87}$ & $41.65_{40.99}^{43.67}$ & & \\
\hline \multirow[t]{3}{*}{ ESO 195-IG21 } & XMM-Newton & 0554500201 & $43.46_{43.41}^{43.50}$ & $43.31_{43.30}^{43.32}$ & $\begin{array}{l}42.83_{42.70}^{42.93} \\
\end{array}$ & $43.32_{43.31}^{43.34}$ \\
\hline & Chandra $\left(2^{\prime \prime}\right)$ & 13898 & $44.41_{44.22}^{44.55}$ & $42.88_{42.31}^{43.29}$ & $41.08_{40.99}^{41.43}$ & $41.76_{41.62}^{41.87}$ \\
\hline & Chandra $\left(20^{\prime \prime}\right)$ & 13898 & $42.24_{42.06}^{42.37}$ & $42.74 \begin{array}{c}43.48 \\
42.25\end{array}$ & & \\
\hline \multirow[t]{2}{*}{ ESO 113-G10 } & XMM-Newton & 0103861601 & $42.67 \begin{array}{r}42.76 \\
42.55\end{array}$ & $42.60_{42.21}^{42.80}$ & $43.03_{43.01}^{43.05}$ & $42.66_{42.66}^{42.67}$ \\
\hline & XMM-Newton & 0301890101 & $43.11_{43.11}^{43.11}$ & $42.76_{42.75}^{42.76}$ & $43.11_{43.10}^{43.12}$ & $42.74 \begin{array}{r}42.74 \\
42.73\end{array}$ \\
\hline \multirow[t]{6}{*}{ NGC 526A } & XMM-Newton & 0109130201 & $42.69_{42.69}^{42.70}$ & $43.17_{43.16}^{43.17}$ & $\begin{array}{l}42.74_{42.74}^{42.75} \\
\end{array}$ & $43.16_{43.15}^{43.16}$ \\
\hline & XMM-Newton & 0150940101 & $42.88_{42.88}^{42.88}$ & $43.29_{43.29}^{43.30}$ & $42.89_{42.88}^{42.89}$ & $43.30_{43.29}^{43.30}$ \\
\hline & XMM-Newton & 0721730301 & $42.95_{42.94}^{42.95}$ & $43.35_{43.35}^{43.36}$ & $42.94_{42.94}^{42.94}$ & $43.35_{43.35}^{43.35}$ \\
\hline & XMM-Newton & 0721730401 & $43.03_{43.03}^{43.03}$ & 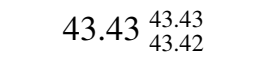 & $43.02_{43.02}^{43.02}$ & $43.43_{43.43}^{43.43}$ \\
\hline & Chandra (4") & 342 & $42.55_{42.50}^{42.59}$ & 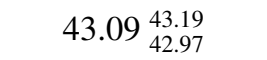 & & \\
\hline & Chandra $\left(35^{\prime \prime}\right)$ & 342 & $41.87_{41.84}^{41.90}$ & $42.58 \begin{array}{r}42.62 \\
42.55\end{array}$ & & \\
\hline \multirow[t]{2}{*}{ MARK 609} & $X M M-N e w t o n$ & 0103861001 & $42.55_{42.54}^{42.56}$ & $42.67_{42.64}^{42.69}$ & $42.58_{42.57}^{42.59}$ & $42.72 \begin{array}{c}42.74 \\
42.71\end{array}$ \\
\hline & XMM-Newton & 0402110201 & $42.50_{42.49}^{42.51}$ & $42.69_{42.68}^{42.71}$ & $42.52_{42.51}^{42.53}$ & $42.66_{42.65}^{42.68}$ \\
\hline \multirow[t]{13}{*}{ NGC 1365} & $X M M-N e w t o n$ & 0151370101 & $41.72_{41.71}^{41.73}$ & $41.86_{41.84}^{41.87}$ & $41.48_{41.47}^{41.49}$ & $41.78_{41.77}^{41.79}$ \\
\hline & $X M M-N e w t o n$ & 0151370201 & $40.97_{40.95}^{40.99}$ & $41.08_{41.04}^{41.12}$ & & \\
\hline & $X M M-N e w t o n$ & 0151370701 & $41.94_{41.93}^{41.95}$ & $41.97 \begin{array}{r}41.99 \\
41.96\end{array}$ & $40.45_{40.44}^{40.47}$ & $42.82_{42.80}^{42.83}$ \\
\hline & $X M M-N e w t o n$ & 0205590301 & $42.11_{42.11}^{42.12}$ & $42.02 \begin{array}{r}42.03 \\
42.02\end{array}$ & $41.62_{41.62}^{41.63}$ & $41.93_{41.92}^{41.93}$ \\
\hline & $X M M-N e w t o n$ & 0205590401 & $41.83_{41.83}^{41.84}$ & $41.90 \begin{array}{r}41.91 \\
41.89\end{array}$ & $39.98 \begin{array}{r}39.99 \\
39.98\end{array}$ & $39.95_{39.94}^{39.96}$ \\
\hline & XMM-Newton & 0505140201 & $41.37_{41.37}^{41.38}$ & $41.33 \begin{array}{r}41.34 \\
41.32\end{array}$ & & \\
\hline & XMM-Newton & 0505140401 & $41.60_{41.60}^{41.60}$ & $41.59_{41.58}^{41.59}$ & & \\
\hline & XMM-Newton & 0505140501 & $41.50_{41.49}^{41.50}$ & $41.49_{41.48}^{41.50}$ & & \\
\hline & XMM-Newton & 0692840201 & $41.97_{41.97}^{41.98}$ & $41.98_{41.97}^{41.98}$ & $40.38 \underset{40.38}{40.38}$ & $41.50_{41.50}^{41.51}$ \\
\hline & XMM-Newton & 0692840301 & $41.54_{41.54}^{41.54}$ & $42.00 \begin{array}{r}42.00 \\
42.00\end{array}$ & & \\
\hline & XMM-Newton & 0692840401 & $41.71_{41.67}^{41.74}$ & $42.00_{41.98}^{42.01}$ & & \\
\hline & XMM-Newton & 0692840501 & $41.75_{41.74}^{41.75}$ & $41.99 \begin{array}{l}41.99 \\
41.98\end{array}$ & $40.76_{40.76}^{40.76}$ & $41.90_{41.90}^{41.90}$ \\
\hline & $X M M-N e w t o n\left(2^{\prime \prime}\right)$ & 0205590401 & & & $42.10_{42.02}^{42.17}$ & $41.94_{41.92}^{41.97}$ \\
\hline
\end{tabular}

Notes. Columns 4 and 5: soft and hard intrinsic luminosities for individual fits; Columns 6 and 7: soft and hard intrinsic luminosities for simultaneous fitting. Blanks mean observations that are not used for the simultaneous fittings. 
Table A.3. continued.

\begin{tabular}{|c|c|c|c|c|c|c|}
\hline \multirow{3}{*}{$\begin{array}{c}\text { Name } \\
(1)\end{array}$} & \multirow{3}{*}{$\begin{array}{c}\text { Satellite } \\
\text { (2) }\end{array}$} & \multirow{3}{*}{$\begin{array}{l}\text { ObsID } \\
\text { (3) }\end{array}$} & \multicolumn{2}{|c|}{ Individual } & \multicolumn{2}{|c|}{ Simultaneous } \\
\hline & & & $\log (L(0.5-2 \mathrm{keV}))$ & $\log (L(2-10 \mathrm{keV}))$ & $\log (L(0.5-2 \mathrm{keV}))$ & $\log (L(2-10 \mathrm{keV}))$ \\
\hline & & & (4) & (5) & (6) & (7) \\
\hline & Chandra $\left(2^{\prime \prime}\right)$ & 6869 & $41.45_{41.41}^{41.49}$ & $41.86_{41.81}^{41.90}$ & $39.69_{38.94}^{39.95}$ & $41.31_{41.27}^{41.34}$ \\
\hline & Chandra $\left(20^{\prime \prime}\right)$ & 6869 & $41.93_{41.92}^{41.95}$ & $42.03_{42.01}^{42.05}$ & & \\
\hline \multirow[t]{2}{*}{ NGC 2617} & XMM-Newton & 0701981601 & $43.06_{43.05}^{43.06}$ & $43.12_{43.11}^{43.12}$ & $43.08_{43.08}^{43.08}$ & $43.10_{43.10}^{43.10}$ \\
\hline & XMM-Newton & 0701981901 & $43.46_{43.46}^{43.46}$ & $43.42_{43.42}^{43.43}$ & $43.35_{43.34}^{43.35}$ & $43.36_{43.36}^{43.37}$ \\
\hline \multirow[t]{2}{*}{ MARK 1218} & XMM-Newton & 0302260201 & $42.09 \begin{array}{r}42.11 \\
42.08\end{array}$ & $42.70_{42.68}^{42.72}$ & $42.04_{42.02}^{42.05}$ & $42.73_{42.71}^{42.75}$ \\
\hline & $X M M-N e w t o n$ & 0302260401 & $41.56_{41.53}^{41.59}$ & $42.35_{42.21}^{43.12}$ & $41.60_{41.57}^{41.63}$ & $42.29_{42.26}^{42.33}$ \\
\hline \multirow[t]{6}{*}{ NGC 2992} & $X M M-N e w t o n$ & 0654910501 & $41.83_{41.83}^{41.84}$ & $42.23_{42.23}^{42.24}$ & $41.79_{41.79}^{41.79}$ & $42.24_{42.24}^{42.24}$ \\
\hline & XMM-Newton & 0654910601 & $41.35_{41.34}^{41.35}$ & $41.84_{41.83}^{41.84}$ & $41.37_{41.36}^{41.37}$ & $41.83_{41.82}^{41.84}$ \\
\hline & $X M M-N e w t o n$ & 0654910701 & $41.34_{41.34}^{41.35}$ & $41.83_{41.83}^{41.84}$ & $41.37_{41.36}^{41.37}$ & $41.83_{41.83}^{41.84}$ \\
\hline & $X M M-N e w t o n$ & 0654910901 & $41.07_{41.07}^{41.08}$ & $41.60_{41.59}^{41.60}$ & $41.11_{41.11}^{41.12}$ & $41.59_{41.58}^{41.60}$ \\
\hline & $X M M-N e w t o n$ & 0654911001 & $41.38_{41.38}^{41.39}$ & $41.88_{41.87}^{41.88}$ & $41.42_{41.41}^{41.42}$ & $41.88_{41.87}^{41.88}$ \\
\hline & XMM-Newton & 0701780101 & $41.92_{41.91}^{41.92}$ & $42.32_{42.31}^{42.33}$ & $41.88_{41.87}^{41.89}$ & $42.33_{42.32}^{42.33}$ \\
\hline \multirow[t]{3}{*}{ POX 52} & XMM-Newton & 0302420101 & $41.55_{41.53}^{41.57}$ & $41.74_{41.72}^{41.76}$ & $41.89_{41.87}^{41.92}$ & $41.76_{41.74}^{41.78}$ \\
\hline & Chandra $\left(2^{\prime \prime}\right)$ & 5736 & $42.26_{42.25}^{42.27}$ & $41.92 \begin{array}{r}42.78 \\
41.89\end{array}$ & $41.88_{41.86}^{41.89}$ & $41.73_{41.71}^{41.75}$ \\
\hline & Chandra $\left(20^{\prime \prime}\right)$ & 5736 & $42.29_{42.28}^{42.30}$ & $41.94_{41.90}^{41.98}$ & & \\
\hline \multirow[t]{3}{*}{ NGC 4138} & $X M M-N e w t o n$ & 0112551201 & - & $41.47_{41.46}^{41.49}$ & - & $41.48_{41.46}^{41.49}$ \\
\hline & Chandra $\left(2^{\prime \prime}\right)$ & 3994 & - & $41.54_{41.36}^{41.66}$ & - & $41.58_{41.27}^{41.76}$ \\
\hline & Chandra $\left(25^{\prime \prime}\right)$ & 3994 & - & $41.54_{41.42}^{41.64}$ & & \\
\hline \multirow[t]{7}{*}{ NGC 4395} & Chandra & 5302 & $39.37_{39.37}^{39.37}$ & $39.96_{39.96}^{39.96}$ & $39.50_{39.46}^{39.54}$ & $39.94 \begin{array}{l}39.96 \\
39.92\end{array}$ \\
\hline & Chandra & 5301 & $39.65_{39.65}^{39.65}$ & $39.93_{39.93}^{39.93}$ & $39.50_{39.47}^{39.53}$ & $39.94 \begin{array}{l}39.96 \\
39.92\end{array}$ \\
\hline & $X M M-N e w t o n$ & 0112521901 & $39.76_{39.73}^{39.78}$ & $40.33_{40.32}^{40.34}$ & $39.75_{39.73}^{39.76}$ & $40.34_{40.33}^{40.35}$ \\
\hline & XMM-Newton & 0744010101 & $39.69_{39.67}^{39.70}$ & $40.23_{40.22}^{40.23}$ & $39.63_{39.62}^{39.64}$ & $40.22_{40.21}^{40.23}$ \\
\hline & $X M M-$ Newton & 0744010201 & $39.65_{39.63}^{39.67}$ & $40.28_{40.27}^{40.29}$ & $39.68_{39.67}^{39.70}$ & $40.28_{40.27}^{40.29}$ \\
\hline & XMM-Newton & 0112521901 & & & $39.94_{39.91}^{39.96}$ & $40.38_{40.34}^{40.42}$ \\
\hline & Chandra & 5301 & & & $39.53_{39.51}^{39.56}$ & $39.93_{39.89}^{39.97}$ \\
\hline \multirow[t]{3}{*}{ NGC 4565} & XMM-Newton & 0112550301 & $39.55_{39.53}^{39.57}$ & $39.76_{39.87}^{40.72}$ & $39.53_{39.51}^{39.55}$ & $39.70_{39.67}^{39.74}$ \\
\hline & Chandra $\left(2^{\prime \prime}\right)$ & 3950 & 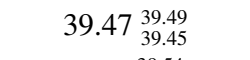 & $40.21_{40.02}^{40.72}$ & $39.48_{39.46}^{39.50}$ & $39.60_{39.56}^{39.64}$ \\
\hline & Chandra $\left(20^{\prime \prime}\right)$ & 3950 & $39.52_{39.50}^{39.54}$ & $39.66_{39.12}^{40.39}$ & & \\
\hline \multirow[t]{4}{*}{ MARK 883} & XMM-Newton & 0302260101 & $42.42_{42.41}^{42.43}$ & $42.73_{42.71}^{42.75}$ & $42.42_{42.41}^{42.43}$ & $42.72_{42.70}^{42.73}$ \\
\hline & XMM-Newton & 0302260701 & $42.42_{42.41}^{42.43}$ & $42.71_{42.69}^{42.73}$ & $42.42_{42.41}^{42.43}$ & $42.71_{42.70}^{42.72}$ \\
\hline & XMM-Newton & 0302261001 & $42.56_{42.55}^{42.57}$ & $42.80_{42.78}^{42.81}$ & $42.52_{42.51}^{42.53}$ & $42.81_{42.80}^{42.83}$ \\
\hline & XMM-Newton & 0652550201 & $42.27_{42.27}^{42.28}$ & $42.59_{42.58}^{42.60}$ & $42.28_{42.28}^{42.29}$ & $42.58_{42.57}^{42.59}$ \\
\hline \multirow[t]{2}{*}{ IRAS 20051-1117 } & XMM-Newton & 0044350201 & $42.45_{42.44}^{42.46}$ & $42.62_{42.60}^{42.65}$ & $42.46_{42.45}^{42.47}$ & $42.60_{42.58}^{42.62}$ \\
\hline & XMM-Newton & 0044350501 & $42.30_{42.29}^{42.31}$ & $42.46_{42.44}^{42.48}$ & $42.31_{42.30}^{42.32}$ & $42.45_{42.44}^{42.47}$ \\
\hline
\end{tabular}


Table A.4. Statistics of the light curves.

\begin{tabular}{|c|c|c|c|c|c|c|}
\hline $\begin{array}{c}\text { Name } \\
(1)\end{array}$ & $\begin{array}{l}\text { ObsID } \\
(2)\end{array}$ & $\begin{array}{c}\text { Energy } \\
\text { (3) }\end{array}$ & $\begin{array}{c}\chi^{2} / \text { d.o.f. } \\
\text { (4) }\end{array}$ & $\begin{array}{c}\text { Prob. }(\%) \\
(5)\end{array}$ & $\begin{array}{c}\sigma_{\mathrm{NXS}}^{2} \\
(6)\end{array}$ & $\begin{array}{c}\left\langle\sigma_{\mathrm{NXS}}^{2}\right\rangle \\
(7)\end{array}$ \\
\hline \multirow[t]{6}{*}{ ESO 113-G10 } & 0301890101 & $0.5-10(1)$ & $1194.3 / 32$ & 100 & $0.0186_{-}^{+} 0.0003$ & $0.01856_{-}^{+} 0.00005$ \\
\hline & & $0.5-10(2)$ & $1703.8 / 39$ & 100 & $0.0185_{-}^{+} 0.0003$ & \\
\hline & & $0.5-2(1)$ & $1014.3 / 32$ & 100 & $0.0185_{-}^{+} 0.0003$ & $0.0183_{-}^{+} 0.0002$ \\
\hline & & $0.5-2(2)$ & $1419.7 / 39$ & 100 & $0.0181_{-}^{+} 0.0003$ & \\
\hline & & $2-10(1)$ & $222.5 / 32$ & 100 & $0.019_{-}^{+} 0.001$ & $0.020_{-}^{+} 0.001$ \\
\hline & & $2-10(2)$ & $333.9 / 39$ & 100 & $0.021_{-}^{+} 0.001$ & \\
\hline \multirow[t]{9}{*}{ NGC 526A } & 0150940101 & $0.5-10$ & $228.9 / 28$ & 100 & $0.0025_{-}^{+} 0.0001$ & \\
\hline & & $0.5-2$ & $130.6 / 28$ & 100 & $0.0038_{-}^{+} 0.0003$ & \\
\hline & & $2-10$ & $128.4 / 28$ & 100 & $0.0018_{-}^{+} 0.0001$ & \\
\hline & 0721730301 & $0.5-10$ & $109.3 / 33$ & 100 & $0.00068_{-}^{+} 0.00007$ & \\
\hline & & $0.5-2$ & $66.1 / 33$ & 100 & $0.0009_{-}^{+} 0.0002$ & \\
\hline & & $2-10$ & $81.3 / 33$ & 100 & $0.0006_{-}^{+} 0.0001$ & \\
\hline & 0721730401 & $0.5-10$ & $376.2 / 38$ & 100 & $0.00241_{-}^{+} 0.00006$ & \\
\hline & & $0.5-2$ & $75.4 / 38$ & 100 & $0.0008_{-}^{+} 0.0002$ & \\
\hline & & $2-10$ & $341.1 / 38$ & 100 & $0.00321_{-}^{+} 0.00009$ & \\
\hline \multirow[t]{27}{*}{ NGC 1365} & 0205590301 & $0.5-10$ & $1012.7 / 37$ & 100 & $0.0206_{-}^{+} 0.0004$ & \\
\hline & & $0.5-2$ & $33.3 / 37$ & 36 & $<0.0014$ & \\
\hline & & $2-10$ & $1464.2 / 37$ & 100 & $0.0412_{-}^{+} 0.0009$ & \\
\hline & 0505140201 & $0.5-10$ & $41.3 / 27$ & 99 & $0.0014_{-}^{+} 0.0007$ & \\
\hline & & $0.5-2$ & $37.2 / 27$ & 99 & $0.0013_{-}^{+} 0.0009$ & \\
\hline & & $2-10$ & $18.8 / 27$ & 12 & $0.004_{-}^{+} 0.004$ & \\
\hline & 0505140401 & $0.5-10(1)$ & $55.3 / 25$ & 100 & $0.0030_{-}^{+} 0.0007$ & $0.0020_{-}^{+} 0.0013$ \\
\hline & & $0.5-10(2)$ & $18.7 / 36$ & 1 & $<0.0014$ & \\
\hline & & $0.5-2(1)$ & $25.6 / 25$ & 57 & $0.0002_{-}^{+} 0.0009$ & $0.0001_{-}^{+} 0.0001$ \\
\hline & & $0.5-2(2)$ & $31.6 / 36$ & 32 & $<0.0017$ & \\
\hline & & $2-10(1)$ & $67.6 / 25$ & 100 & $0.018_{-}^{+} 0.003$ & $0.0012_{-}^{+} 0.0008$ \\
\hline & & $2-10(2)$ & $32.2 / 36$ & 35 & $<0.0068$ & \\
\hline & 0505140501 & $0.5-10$ & $53.3 / 30$ & 100 & $0.0020_{-}^{+} 0.0007$ & \\
\hline & & $0.5-2$ & $45.0 / 30$ & 99 & $0.0016_{-}^{+} 0.0008$ & \\
\hline & & $2-10$ & $24.1 / 30$ & 33 & $<0.0077$ & \\
\hline & 0692840201 & $0.5-10(1)$ & $634.3 / 40$ & 100 & $0.0183_{-}^{+} 0.0005$ & $0.0107_{-}^{+} 0.0035$ \\
\hline & & $0.5-10(2)$ & $384.1 / 40$ & 100 & $0.0105_{-}^{+} 0.0003$ & \\
\hline & & $0.5-10(3)$ & $228.6 / 38$ & 100 & $0.0063_{-}^{+} 0.0003$ & \\
\hline & & $0.5-2(1)$ & $52.6 / 40$ & 99 & $0.0010_{-}^{+} 0.0007$ & $0.0006_{-}^{+} 0.0003$ \\
\hline & & $0.5-2(2)$ & $47.4 / 40$ & 80 & $0.0006_{-}^{+} 0.0007$ & \\
\hline & & $0.5-2(3)$ & $60.0 / 38$ & 100 & $0.0019_{-}^{+} 0.0007$ & \\
\hline & & $2-10(1)$ & $1291.7 / 40$ & 100 & $0.058_{-}^{+} 0.002$ & $0.028_{-}^{+} 0.009$ \\
\hline & & $2-10(2)$ & $630.9 / 40$ & 100 & $0.0281_{-}^{+} 0.0009$ & \\
\hline & & $2-10(3)$ & $352.6 / 38$ & 100 & $0.0178_{-}^{+} 0.0007$ & \\
\hline & 0692840301 & $0.5-10(1)$ & $3511.7 / 38$ & 100 & $0.0426_{-}^{+} 0.0006$ & $0.0417_{-}^{+} 0.0008$ \\
\hline & & $0.5-10(2)$ & $3763.3 / 40$ & 100 & $0.0409_{-}^{+} 0.0005$ & \\
\hline & & $0.5-2(1)$ & $659.6 / 38$ & 100 & $0.0257_{-}^{+} 0.0007$ & $0.0262_{-}^{+} 0.0005$ \\
\hline
\end{tabular}

Notes. (Column 1) name, (Col. 2) obsID, (Col. 3) energy band in keV, (Cols. 4 and 5) $\chi^{2} /$ d.o.f. and the probability of being variable in the $0.5-$ $10.0 \mathrm{keV}$ energy band of the total light curve, (Col. 6) normalized excess variance, $\sigma_{\mathrm{NXS}}^{2}$, and (Col. 8) the mean value of the normalized excess variance, $\left\langle\sigma_{\mathrm{NXS}}^{2}\right\rangle$, for each light curve and energy band. 
Table A.4. continued.

\begin{tabular}{|c|c|c|c|c|c|c|}
\hline $\begin{array}{c}\text { Name } \\
\text { (1) }\end{array}$ & $\begin{array}{c}\text { ObsID } \\
(2)\end{array}$ & $\begin{array}{c}\text { Energy } \\
\text { (3) }\end{array}$ & $\begin{array}{c}\chi^{2} / \text { d.o.f. } \\
\text { (4) }\end{array}$ & $\begin{array}{c}\text { Prob. }(\%) \\
(5)\end{array}$ & $\begin{array}{c}\sigma_{\mathrm{NXS}}^{2} \\
(6)\end{array}$ & $\begin{array}{c}\left\langle\sigma_{\mathrm{NXS}}^{2}\right\rangle \\
(7)\end{array}$ \\
\hline & & $0.5-2(2)$ & $791.5 / 40$ & 100 & $0.0267_{-}^{+} 0.0007$ & \\
\hline & & $2-10(1)$ & $3229.5 / 38$ & 100 & $0.0538_{-}^{+} 0.0009$ & $0.052_{-}^{+} 0.002$ \\
\hline & & $2-10(2)$ & $3175.1 / 40$ & 100 & $0.0495_{-}^{+} 0.0008$ & \\
\hline & 0692840401 & $0.5-10(1)$ & $5994.8 / 37$ & 100 & $0.090_{-}^{+} 0.001$ & $0.06_{-}^{+} 0.02$ \\
\hline & & $0.5-10(2)$ & $5409.8 / 36$ & 100 & $0.0453_{-}^{+} 0.0005$ & \\
\hline & & $0.5-2(1)$ & $1458.4 / 37$ & 100 & $0.067_{-}^{+} 0.002$ & $0.058_{-}^{+} 0.006$ \\
\hline & & $0.5-2(2)$ & $2447.0 / 36$ & 100 & $0.054_{-}^{+} 0.001$ & \\
\hline & & $2-10(1)$ & $4977.2 / 37$ & 100 & $0.106_{-}^{+} 0.002$ & $0.06_{-}^{+} 0.02$ \\
\hline & & $2-10(2)$ & $3164.0 / 36$ & 100 & $0.0430_{-}^{+} 0.0006$ & \\
\hline & 0692840501 & $0.5-10(1)$ & $6872.7 / 36$ & 100 & $0.145_{-}^{+} 0.002$ & $0.03_{-}^{+} 0.02$ \\
\hline & & $0.5-10(2)$ & $1125.4 / 38$ & 100 & $0.0276_{-}^{+} 0.0006$ & \\
\hline & & $0.5-10(3)$ & $504.2 / 39$ & 100 & $0.0115_{-}^{+} 0.0003$ & \\
\hline & & $0.5-2(1)$ & $559.1 / 36$ & 100 & $0.054_{-}^{+} 0.002$ & $0.01_{-}^{+} 0.02$ \\
\hline & & $0.5-2(2)$ & $21.9 / 38$ & 2 & $<0.0014$ & \\
\hline & & $0.5-2(3)$ & $24.9 / 39$ & 5 & $<0.0014$ & \\
\hline & & $2-10(1)$ & $7598.8 / 36$ & 100 & $0.192_{-}^{+} 0.004$ & $0.05_{-}^{+} 0.03$ \\
\hline & & $2-10(2)$ & $1585.9 / 38$ & 100 & $0.059_{-}^{+} 0.001$ & \\
\hline & & $2-10(3)$ & $753.4 / 39$ & 100 & $0.0271_{-}^{+} 0.0007$ & \\
\hline \multirow[t]{3}{*}{ NGC 2617} & 0701981601 & $0.5-10$ & $128.7 / 31$ & 100 & $0.00046_{-}^{+} 0.00004$ & \\
\hline & & $0.5-2$ & $138.3 / 31$ & 100 & $0.00065_{-}^{+} 0.00005$ & \\
\hline & & $2-10$ & $27.1 / 31$ & 73 & $<0.0003$ & \\
\hline \multirow[t]{15}{*}{ NGC 2992} & 0654910501 & $0.5-10$ & $41.2 / 40$ & 58 & $0.00003_{-}^{+} 0.00014$ & \\
\hline & & $0.5-2$ & $44.9 / 40$ & 73 & $0.0002_{-}^{+} 0.0003$ & \\
\hline & & $2-10$ & $27.6 / 40$ & 7 & $0.00031_{-}^{+} 0.0002$ & \\
\hline & 0654910601 & $0.5-10$ & $29.4 / 22$ & 87 & $0.0006_{-}^{+} 0.0005$ & \\
\hline & & $0.5-2$ & $16.2 / 22$ & 19 & $<0.0024$ & \\
\hline & & $2-10$ & $28.2 / 22$ & 83 & $0.0011_{-}^{+} 0.0009$ & \\
\hline & 0654910701 & $0.5-10$ & $70.4 / 36$ & 100 & $0.0015_{-}^{+} 0.0004$ & \\
\hline & & $0.5-2$ & $36.2 / 36$ & 54 & $0.00002_{-}^{+} 0.00078$ & \\
\hline & & $2-10$ & $61.8 / 36$ & 100 & $0.0021_{-}^{+} 0.0007$ & \\
\hline & 0654910901 & $0.5-10$ & $24.9 / 29$ & 32 & $<0.0017$ & \\
\hline & & $0.5-2$ & $25.7 / 29$ & 36 & $<0.0033$ & \\
\hline & & $2-10$ & $25.6 / 29$ & 35 & $<0.0035$ & \\
\hline & 0654911001 & $0.5-10$ & $61.1 / 32$ & 100 & $0.0014_{-}^{+} 0.0004$ & \\
\hline & & $0.5-2$ & $45.2 / 32$ & 99 & $0.0015_{-}^{+} 0.0008$ & \\
\hline & & $2-10$ & $50.9 / 32$ & 99 & $0.0017_{-}^{+} 0.0007$ & \\
\hline \multirow[t]{6}{*}{ POX 52} & 0302420101 & $0.5-10(1)$ & $59.5 / 23$ & 100 & $0.09_{-}^{+} 0.02$ & $0.05_{-}^{+} 0.03$ \\
\hline & & $0.5-10(2)$ & $50.9 / 33$ & 99 & $0.03_{-}^{+} 0.01$ & \\
\hline & & $0.5-2(1)$ & $60.6 / 23$ & 100 & $0.20_{-}^{+} 0.06$ & $0.08_{-}^{+} 0.08$ \\
\hline & & $0.5-2(2)$ & $38.2 / 33$ & 76 & $0.02_{-}^{+} 0.03$ & \\
\hline & & $2-10(1)$ & $34.6 / 23$ & 95 & $0.05_{-}^{+} 0.03$ & $0.03_{-}^{+} 0.01$ \\
\hline & & $2-10(2)$ & $45.8 / 33$ & 99 & $0.02_{-}^{+} 0.02$ & \\
\hline \multirow[t]{3}{*}{ NGC 4395} & 0744010101 & $0.5-10$ & $782.1 / 40$ & 100 & $0.069_{-}^{+} 0.003$ & \\
\hline & & $0.5-2$ & $83.7 / 40$ & 100 & $0.045_{-}^{+} 0.01$ & \\
\hline & & $2-10$ & $815.1 / 40$ & 100 & $0.077_{-}^{+} 0.003$ & \\
\hline NGC 4565 & 3950 & $0.5-10$ & $30.1 / 40$ & 13 & $<0.0161$ & \\
\hline
\end{tabular}


Table A.4. continued.

\begin{tabular}{ccccccc}
\hline \hline Name & ObsID & Energy & $\chi^{2} /$ d.o.f. & Prob.(\%) & $\sigma_{\text {NXs }}^{2}$ & $\left\langle\sigma_{\text {NXS }}^{2}\right\rangle$ \\
$(1)$ & $(2)$ & $(3)$ & $(4)$ & $(5)$ & $(6)$ & $(7)$ \\
\hline & $0.5-2$ & $43.5 / 40$ & 68 & $0.004_{-}^{+} 0.001$ & \\
& $2-10$ & $40.0 / 40$ & 53 & $<0.0548$ & \\
\hline
\end{tabular}

Table A.5. Classification of the sample objects on the basis of the nuclear X-ray obscuration (and its variability).

\begin{tabular}{|c|c|c|c|c|c|c|c|}
\hline Name & ObsID & $\Gamma$ & $\begin{array}{c}E W \\
(\mathrm{keV})\end{array}$ & $F_{x} / F_{[\mathrm{OIII}]}$ & $\begin{array}{l}\text { Ref. }^{1} \\
{[\mathrm{OIII}]}\end{array}$ & CT? & Classification \\
\hline (1) & (2) & (3) & (4) & (5) & (6) & (7) & (8) \\
\hline ESO 540-G01 & 0044350101 & $1.44_{1.07}^{2.32}$ & $<0.48$ & - & - & $x$ & Compton-thin \\
\hline \multirow[t]{2}{*}{ ESO 195-IG21 } & 0554500201 & $1.29_{1.06}^{1.52}$ & $0.11_{0.08}^{0.15}$ & - & - & $x$ & Compton-thin \\
\hline & 13898 & $2.06_{0.87}^{2.57}$ & $0.51_{0.23}^{0.78}$ & - & & $x$ & \\
\hline \multirow[t]{2}{*}{ ESO $113-G 10$} & 0103861601 & $1.62_{1.38}^{1.89}$ & $<0.18$ & - & - & $x$ & Compton-thin \\
\hline & 0301890101 & $1.80_{1.75}^{1.87}$ & $<0.04$ & - & & $x$ & \\
\hline \multirow[t]{5}{*}{ NGC 526A } & 0109130201 & $1.30_{1.24}^{1.41}$ & $0.08_{0.05}^{0.11}$ & 75.4 & 1 & $x$ & Compton-thin \\
\hline & 0150940101 & $1.55_{1.48}^{1.61}$ & $0.06_{0.05}^{0.07}$ & 99.4 & & $x$ & \\
\hline & 0721730301 & $1.48_{1.43}^{1.53}$ & $0.07_{0.06}^{0.07}$ & 114.2 & & $x$ & \\
\hline & 0721730401 & $1.58_{1.52}^{1.63}$ & $0.06_{0.05}^{0.07}$ & 137.3 & & $x$ & \\
\hline & 342 & $<0.53$ & $<0.23$ & 19.4 & & $x$ & \\
\hline \multirow[t]{2}{*}{ MARK 609} & 0103861001 & $1.59_{1.22}^{1.86}$ & $<0.16$ & 31.1 & 2 & $x$ & Compton-thin \\
\hline & 0402110201 & $1.25_{1.08}^{1.45}$ & $0.11_{0.03}^{0.19}$ & 32.5 & & $x$ & \\
\hline \multirow[t]{13}{*}{ NGC 1365} & 0151370101 & $1.18_{0.91}^{1.46}$ & $0.20_{0.16}^{0.25}$ & 13.2 & 1 & $x$ & Changing-look \\
\hline & 0151370201 & $<1.36$ & $<0.31$ & 2.2 & & $x$ & \\
\hline & 0151370701 & $2.44_{2.20}^{2.77}$ & $0.14_{0.09}^{0.18}$ & 17.0 & & $x$ & \\
\hline & 0205590301 & $2.88_{2.84}^{2.94}$ & $0.11_{0.10}^{0.12}$ & 19.1 & & $x$ & \\
\hline & 0205590401 & $2.18_{2.08}^{2.37}$ & $0.17_{0.15}^{0.19}$ & 14.5 & & $x$ & \\
\hline & 0505140201 & $<0.39$ & $0.46_{0.41}^{0.52}$ & 3.9 & & $\checkmark$ & \\
\hline & 0505140401 & $<0.09$ & $0.42_{0.39}^{0.54}$ & 7.1 & & $\checkmark$ & \\
\hline & 0505140501 & $<0.17$ & $0.43_{0.38}^{0.58}$ & 5.6 & & $\sqrt{ }$ & \\
\hline & 0692840201 & $2.08_{1.99}^{2.12}$ & $0.12_{0.11}^{0.13}$ & 17.4 & & $x$ & \\
\hline & 0692840301 & $2.40_{2.38}^{2.44}$ & $0.09_{0.08}^{0.10}$ & 18.2 & & $x$ & \\
\hline & 0692840401 & $2.32_{2.31}^{2.38}$ & $0.08_{0.07}^{0.09}$ & 18.2 & & $x$ & \\
\hline & 0692840501 & $2.20_{2.13}^{2.22}$ & $0.09_{0.08}^{0.10}$ & 17.8 & & $x$ & \\
\hline & 6869 & $1.34_{0.96}^{2.08}$ & $0.13_{0.07}^{0.19}$ & 13.2 & & $x$ & \\
\hline \multirow[t]{2}{*}{ NGC 2617} & 0701981601 & $1.60_{1.58}^{1.63}$ & $0.06_{0.05}^{0.07}$ & - & - & $x$ & Compton-thin \\
\hline & 0701981901 & $1.70_{1.67}^{1.74}$ & $0.05_{0.03}^{0.06}$ & - & & $x$ & \\
\hline \multirow[t]{2}{*}{ MARK 1218} & 0302260201 & $1.34_{1.13}^{1.75}$ & $<0.09$ & 18.1 & 2 & $x$ & Compton-thin \\
\hline & 0302260401 & $1.670_{0.74}^{2.99}$ & $<0.20$ & 8.1 & & $x$ & \\
\hline \multirow[t]{2}{*}{ NGC 2992} & 0654910501 & $1.54_{1.46}^{1.63}$ & $0.16_{0.14}^{0.18}$ & 2.2 & 1 & $x$ & Changing-look \\
\hline & 0654910601 & $1.31_{1.20}^{1.45}$ & $0.33_{0.29}^{0.36}$ & 0.9 & & $x$ & \\
\hline
\end{tabular}

Notes. (Column 1) name, (Col. 2) obsID, (Cols. 3 and 4) index of the power law and the equivalent width of the FeK $\alpha$ line from the spectral fit (PL model) in the 3-10 keV energy band, (Col. 5) ratio between the individual hard X-ray luminosity (from Table A.3) and the extinction-corrected [O III] fluxes, (Col. 6) references for the measure of $F_{[\mathrm{OIII}]}$, (Col. 7) classification from the individual observation, (Col. 8) classification of the object, and (Col. 9) slope of the power law at hard energies for Compton-thick candidates from the simultaneous analysis (see Sect. 4.5).

References. (1) Bassani et al. (1999); (2) Dahari \& De Robertis (1988); (3) Whittle (1992); (4) Panessa et al. (2006); and (5) Panessa \& Bassani (2002). 
Table A.5. continued.

\begin{tabular}{|c|c|c|c|c|c|c|c|}
\hline Name & ObsID & $\Gamma$ & $\begin{array}{c}E W \\
(\mathrm{keV})\end{array}$ & $F_{x} / F_{[\mathrm{OIII}]}$ & $\begin{array}{l}\text { Ref. }^{1} \\
{[\mathrm{OIII}]}\end{array}$ & CT? & Classification \\
\hline \multirow[t]{5}{*}{ (1) } & $(2)$ & (3) & (4) & (5) & $(6)$ & (7) & (8) \\
\hline & 0654910701 & $1.26_{1.22}^{1.36}$ & $0.32_{0.29}^{0.34}$ & 0.9 & & $x$ & \\
\hline & 0654910901 & $1.23_{1.05}^{1.42}$ & $0.50_{0.45}^{0.55}$ & 0.5 & & $\checkmark$ & \\
\hline & 0654911001 & $1.30_{1.22}^{1.42}$ & $0.25_{0.22}^{0.28}$ & 1.0 & & $x$ & \\
\hline & 0701780101 & $1.39_{1.33}^{1.54}$ & $0.17_{0.14}^{0.21}$ & 2.8 & & $x$ & \\
\hline \multirow[t]{2}{*}{ POX52 } & 0302420101 & $1.48_{1.14}^{1.88}$ & $<0.11$ & 8.2 & 3 & $x$ & Compton-thin \\
\hline & 5736 & $1.79_{1.44}^{3.23}$ & $<0.45$ & 12.5 & & $x$ & \\
\hline \multirow[t]{2}{*}{ NGC 4138} & 0112551201 & $1.23_{0.98}^{1.49}$ & $0.08_{0.04}^{0.13}$ & 478.6 & 4 & $x$ & Compton-thin \\
\hline & 3994 & $1.29_{0.18}^{1.94}$ & $0.17_{0.04}^{0.30}$ & 478.6 & & $x$ & \\
\hline \multirow[t]{3}{*}{ NGC 4395} & 0112521901 & $1.28_{1.05}^{1.53}$ & $0.11_{0.07}^{0.16}$ & 64.8 & 4 & $x$ & Compton-thin \\
\hline & 5301 & $1.24_{0.80}^{1.65}$ & $<0.11$ & 27.7 & & $x$ & \\
\hline & 5302 & $1.26_{0.64}^{1.67}$ & $<0.10$ & 25.8 & & $x$ & \\
\hline \multirow[t]{2}{*}{ NGC 4565} & 0112550301 & $1.55_{1.08}^{2.49}$ & $<0.71$ & 5.6 & 5 & $x$ & Compton-thin \\
\hline & 3950 & $2.73_{1.51}^{2.95}$ & $0.03_{0.01}^{0.05}$ & 15.4 & & $x$ & \\
\hline \multirow[t]{4}{*}{ MARK 883} & 0302260101 & $1.22_{1.05}^{1.49}$ & $<0.17$ & 27.5 & 2 & $x$ & Compton-thin \\
\hline & 0302260701 & $1.44_{1.26}^{1.65}$ & $0.12_{0.02}^{0.21}$ & 26.2 & & $x$ & \\
\hline & 0302261001 & $1.58_{1.34}^{2.13}$ & $0.10_{0.02}^{0.19}$ & 32.3 & & $x$ & \\
\hline & 0652550201 & $1.43_{1.33}^{1.73}$ & $0.18_{0.11}^{0.24}$ & 19.9 & & $x$ & \\
\hline \multirow{2}{*}{ IRAS 20051-1117 } & 0044350201 & $1.68_{1.36}^{2.41}$ & $0.21_{0.06}^{0.35}$ & 13.8 & & $x$ & Compton-thin \\
\hline & 0044350501 & $1.70_{1.48}^{2.25}$ & $<0.16$ & 9.5 & 5 & $x$ & \\
\hline
\end{tabular}


A\&A 602, A65 (2017)
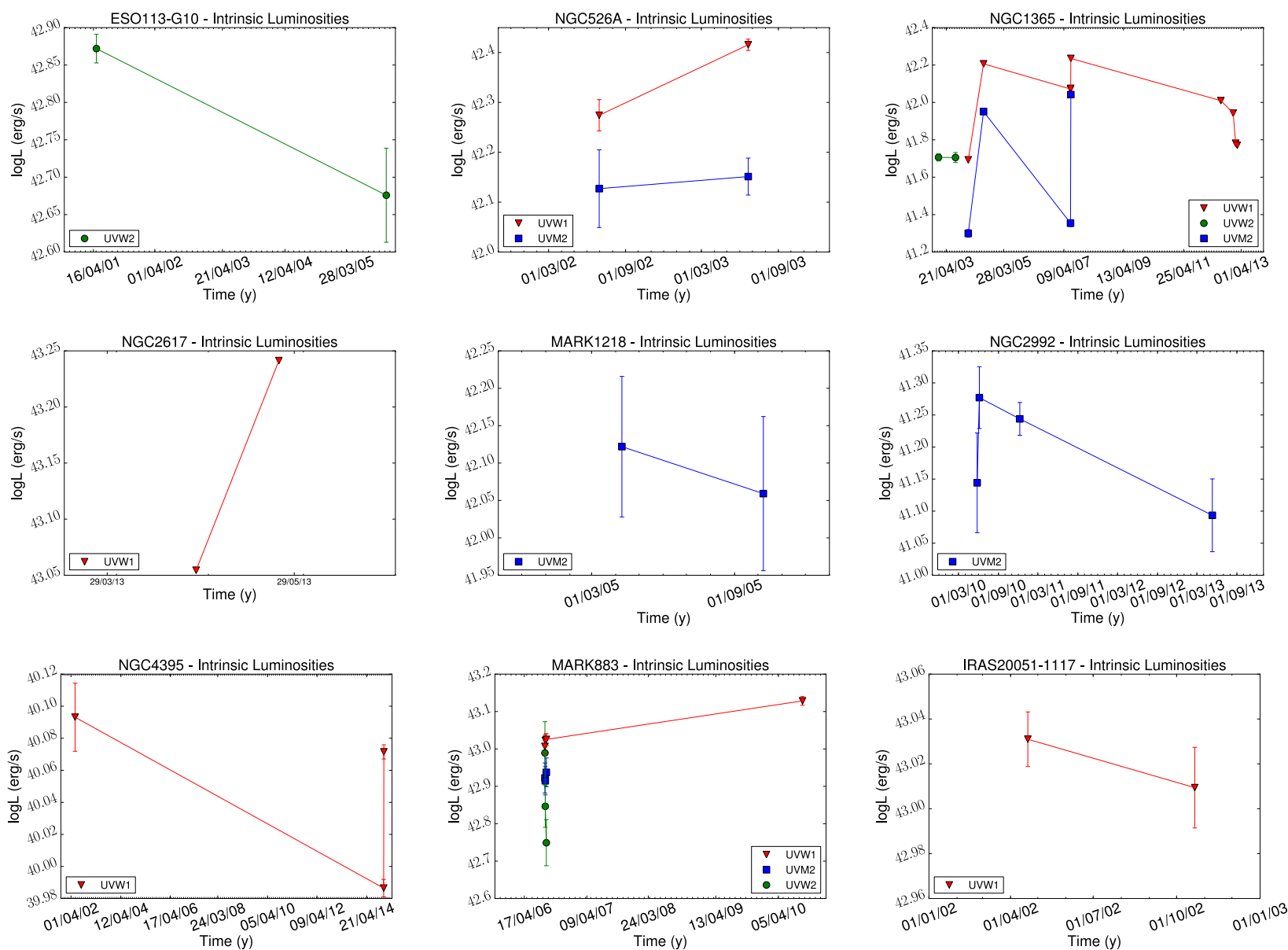

Fig. A.1. UV luminosities obtained from the data with the OM camera onboard XMM-Newton, when available. Different filters have been used; $U V W 1$ (red triangles), $U V W 2$ (green circles), and $U V M 2$ (blue squares). 
L. Hernández-García et al.: X-ray variability in Seyfert 1.8/1.9 galaxies
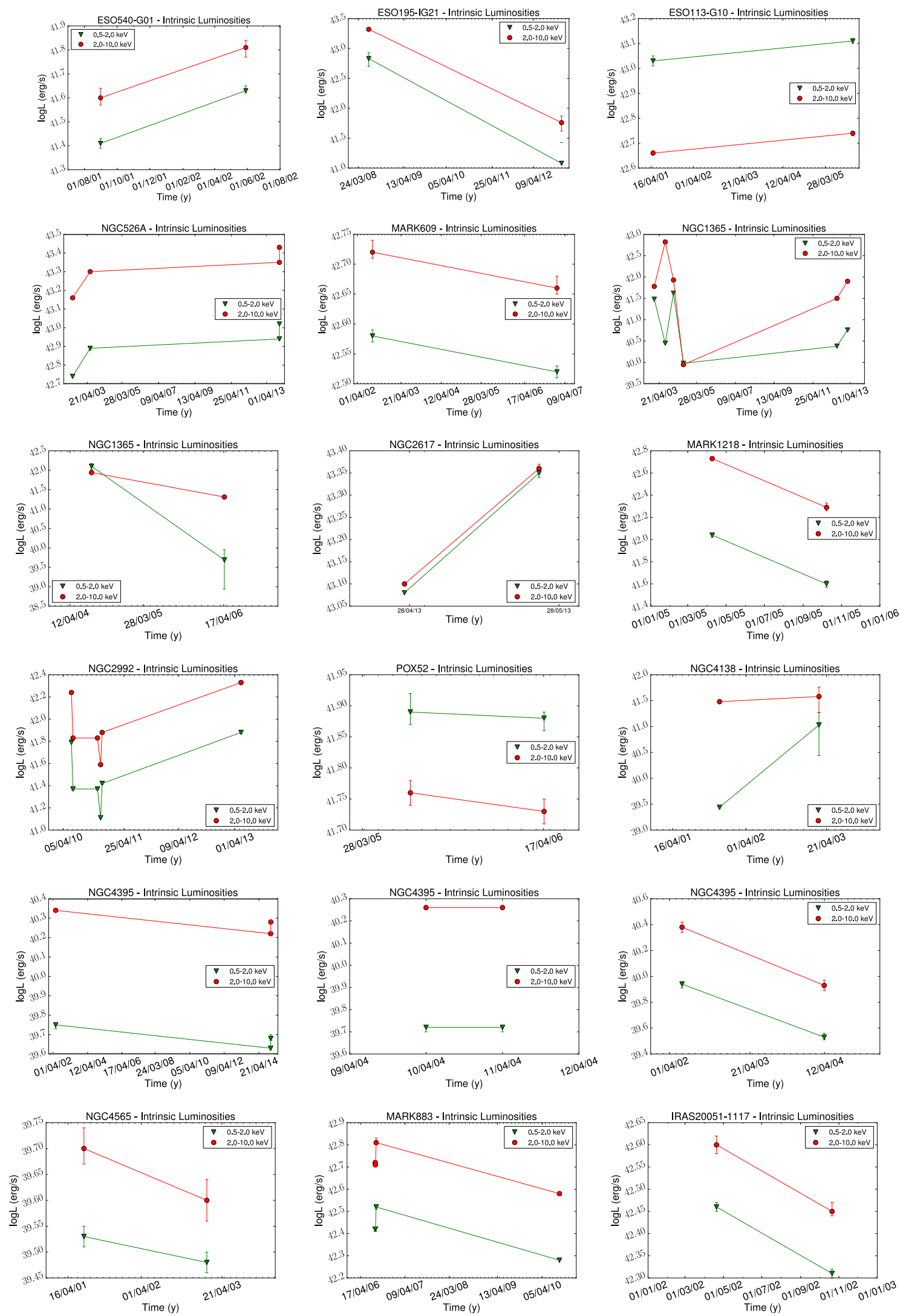

Fig. A.2. X-ray intrinsic luminosities calculated for the soft (0.5-2.0 keV, green triangles) and hard (2.0-10.0 keV, red circles) energies in the simultaneous fits, only for the variable objects. 


\section{Appendix B: Notes and comparisons with previous results for individual objects}

In this appendix we discuss the general characteristics of the galaxies in our sample at different wavelengths, as well as comparisons with previous variability studies. We recall that longterm UV variability and short-term X-ray variations were studied only for some sources (ten and seven sources, see Tables 1 and A.4, respectively), so comparisons are only made in those cases. For the remaining objects, results from other authors are mentioned, when available.

\section{B.1. ESO540-G01}

NGC 540-G01 is the brightest member of the Hickson compact group 4 (Hickson et al. 1989). It was classified as a Seyfert 1.8 using optical data (Coziol et al. 1993), although also as a composite galaxy (Moran et al. 1996). A radio counterpart was detected with VLA data at $1.4 \mathrm{GHz}$ (Condon et al. 1998). It is a source classified as a type 1 AGN showing no X-ray absorption (Panessa et al. 2005).

This galaxy was observed once by Chandra in 2001 and once by XMM-Newton in 2002. Georgantopoulos et al. (2003) studied the Chandra data. They found long-term X-ray flux variations when comparing with previous ROSAT data by a factor of 25 in ten years. When comparing Chandra and XMM-Newton data, the annular region contributed with $21 \%$ in Chandra data, and the best representation is obtained with SMF1 showing variations in $\mathrm{Norm}_{2}(74 \%)$ in one year period. This implies flux variability of $40 \%(38 \%)$ in the soft (hard) energy band.

Georgantopoulos et al. (2003) studied the Chandra light curve and did not find short-term variability.

\section{B.2. ESO 195-IG21}

ESO 195-IG21 was classified as a Seyfert 1.8 using optical data, after its discovery using Swift data (Baumgartner et al. 2008). We did not find data at radio frequencies for this source in the literature.

This galaxy was observed once by XMM-Newton in 2008 and once by Chandra in 2012. Variability studies of this source were not found in the literature. In this case, the annular region contributed with $10 \%$ to the Chandra data. When comparing the data, SMF2 results in the best fit with Norm $_{1}(91 \%)$ and Norm 2 $(98 \%)$ varying in a four years period. This results in flux variability of $98 \%$ (97\%) in the soft (hard) energy band.

\section{B.3. ESO 113-G10}

The nucleus was classified as a Seyfert 1.8 in the optical by Pietsch et al. (1998), who also reported X-ray variations of a factor of three between two ROSAT observations obtained within six months.

ESO 113-G10 was observed twice with XMM-Newton in 2001 and 2005. Long-term X-ray variability studies of these source were not found in the literature. We find that SMF1 with variations in Norm $_{1}(40 \%)$ represents the data best in a four-year period. This implies flux variations of $17 \%$ in both the soft and hard energy bands.

Porquet et al. (2007) found short-term variations in the soft and hard light curve from 2005 in timescales lower than $500 \mathrm{~s}$. These results agree well with those obtained by González-Martín \& Vaughan (2012) and ours, because the analysis of the same light curve resulted in short-term variations of the total, soft, and hard energy bands. Later, Cackett et al. (2013) used the same observation to report a hard and a soft lag at low and high frequencies, respectively, between the $1.5-4.5 \mathrm{keV}$ and $0.3-0.9 \mathrm{keV}$ energy bands.

At UV frequencies, we detected variations in the $U V W 2(8 \sigma)$ filter from the OM.

\section{B.4. NGC 526A}

NGC 526A is the west galaxy in the strongly interacting pair of galaxies in NGC 526 (Mulchaey et al. 1996). It was optically classified as a Seyfert 1.9 (Griffiths et al. 1979; Winkler 1992). A radio counterpart was detected with VLA data at 3.6 and $20 \mathrm{~cm}$ (Nagar et al. 1999).

$\mathrm{X}$-ray flux and spectral variability was detected in this source as observed by HEAO 1, Einstein, EXOSAT and GINGA in timescales of years (Mushotzky 1982; Turner \& Pounds 1989; Polletta et al. 1996).

It has been observed with Chandra five times between 1999 and 2003, and four times with XMM-Newton between 2002 and 2013. Long-term variability analyses using these data are not reported in the current literature. SMF1 is the best representation of the $X M M-N e w t o n$ data presented in this work, with variations in $\mathrm{Norm}_{2}(48 \%)$. This implies a flux variation of $48 \%(46 \%)$ in the soft (hard) energy band in $11 \mathrm{yr}$ period. When comparing with Chandra data (the annular region contributed with $45 \%$ in Chandra data) out method cannot differentiate if variations in $N_{\mathrm{H} 2}\left(\chi_{r}^{2}=1.03\right)$ or variations in $\operatorname{Norm}_{2}\left(\chi_{r}^{2}=1.08\right)$ are preferred in the two-year period, both results being a good option. For this reason we do not report this analysis and we take into account the results from the XMM-Newton data.

González-Martín \& Vaughan (2012) studied the XMM-Newton observation from 2003 and found that it showed short-term variations in the three analyzed energy bands. We studied three XMM-Newton observations (except the one from 2002) and detected short-term variations in all of them and the three energy bands, in good agreement with González-Martín \& Vaughan (2012). In short timescales, Turner et al. (1997) also detected rapid variations using ASCA data. Using RXTE data between 2001 and 2003, Zhang (2011) estimated $\sigma_{\text {NXS }}^{2}=8.16_{-}^{+} 1.81 \times$ $10^{-2}$.

In the 14-195 keV energy band, Soldi et al. (2014) estimated a variability amplitude of $35_{-}^{+} 6 \%$ using data from the $S$ wift/BAT 58-month survey.

UV data from the OM are available in two filters. Variations are detected in the $U V W 1(11 \sigma)$ but not in the $U V M 2(1 \sigma)$ filter.

\section{B.5. MARK 609}

This galaxy was classified as a Seyfert 1.8 by Osterbrock (1981), although it was later classified as a type 2 and 1.5, suggesting that this might be a changing-look candidate (Trippe et al. 2010, and references therein). It has also been proposed to be a "true" Seyfert 2, meaning an AGN that lack circumnuclear obscuration (LaMassa et al. 2011, 2014). A radio counterpart was detected with VLA data at $6 \mathrm{~cm}$ (Ulvestad \& Wilson 1984).

The source was observed twice with XMM-Newton in 2002 and 2007. Variability studies using these data were not found in the literature. We find that SMF1 represents best the data, requiring changes in Norm $_{1}(22 \%)$ within five years. This implies flux variations of $13 \%$ in both the soft and hard energy bands. 


\section{B.6. NGC 1365}

NGC 1365 is a barred spiral galaxy located in the Fornax I cluster. It was classified as a Seyfert 1.8 at optical wavelenghts (Maiolino \& Rieke 1995). This is the prototypical example of a changing-look source, having changed from reflectiondominated to transmission dominated states in different occasions, in good agreement with the results presented here (e.g., Risaliti et al. 2009). A radio counterpart was observed with VLA data (Jorsater \& van Moorsel 1995).

It was observed 13 times with XMM-Newton between 2003 and 2013, and seven times with Chandra between 2002 and 2006.

Risaliti et al. (2005b) studied a Chandra observation from 2002 and three XMM-Newton observations from 2003. They reported variations from Compton-thin to Compton-thick in timescales of six weeks, which were attributed to changes in the absorber, whereas the soft thermal emission and the reflection component remained constant. The same authors also discovered four absorption lines between 6.7 and $8.3 \mathrm{keV}$ (identified them as $\mathrm{Fe} X X V$ and $\mathrm{Fe} X X V I \mathrm{~K} \alpha$ and $\mathrm{K} \beta$ lines) related to absorption by a highly ionized gas (Risaliti et al. 2005a), and detected an X-ray eclipse from clouds in the BLR from the XMM-Newton observation from January 2004 (Risaliti et al. 2009). Guainazzi et al. (2009) analyzed the RGS data and suggested that the relative weakness of the photoionisation might be related to the same material responsible for the X-ray abosrbing column density variations.

Simultaneous XMM-Newton and Suzaku data from 2012 and 2013 were studied by different authors. Risaliti et al. (2013) and Walton et al. (2014) found continuum variations related to absorption from reflection, Parker et al. (2014) reported variability in the absorber and intrinsic to the source using a principal component analysis (PCA), Braito et al. (2014) found absorption variations in timescales of about $100 \mathrm{ks}$ in the $0.3-1.8 \mathrm{keV}$ energy band related to a low ionization zone of a disk wind, and Rivers et al. (2015) reported that changes in this source are mainly due to absorption. Our method does not allow to perform the spectral fit to all the spectra simultaneously $\left(\chi_{r}^{2}>\right.$ 2.5). Instead, we removed the spectra in the Compton-thick state (Risaliti et al. 2005a) and those in a "relativistic" state (Risaliti et al. 2013). Therefore we used six XMM-Newton observations. The best fit resulted from the use of SMF3 with $N_{\mathrm{H} 2}$ $(68 \%)$, Norm $_{2}(33 \%)$, and Norm $1(35 \%)$ varying. These changes imply a change in flux of $81 \%(24 \%)$ in the soft (hard) energy band in a $10 \mathrm{yr}$ period. When comparing Chandra and XMMNewton data, SMF2 was required with variations in $N_{\mathrm{H} 2}(37 \%)$ and $\mathrm{Norm}_{2}(30 \%)$ in a two-year period. The annular region contributed with $53 \%$ in Chandra data.

Connolly et al. (2014) studied 190 observations obtained with Swift between 2006 and 2013. They reported variations in the normalizations of the soft and hard energy bands plus changes in the absorbing material, and interpreted these variations in terms of an AGN wind scenario.

Brenneman et al. (2013) studied three Suzaku observations of NGC 1365 obtained between 2008 and 2010. They reported variations both at short- and long-term, mainly because of absorption and continuum variations.

González-Martín \& Vaughan (2012) studied three XMMNewton observations between 2004 and 2007. They found shortterm variations in the hard and total energy bands of the three observations, whereas the soft band did not show variations. We analyzed two of these light curves and found a good agreement. In total, we were able to study short-term variations in eight observations. We found that variations in the hard and total energy bands are very common in all the observations, but variability in the soft band is observed only in observations from 2012 and 2013. The light curve presented by Connolly et al. (2014) using Swift data also showed short-term variations. They attributed these variations to clouds passing throught the line of sight of the observer. Using the simultaneous XMM-Newton and NuSTAR data, Kara et al. (2015) found a Fe K lag, plus another lag at low frequencies, probably due to absorption variations.

Data at UV frequencies were available from the OM in the three UV filters. Strong variations were detected with the UVWI $(62 \sigma)$ and UVM2 $(76 \sigma)$ filters. Variations were not detected with the $U V W 2(1 \sigma)$ filter, but data were available only in two epochs separated by about seven months.

\section{B.7. NGC 2617}

NGC 2617 is a spiral galaxy. It was optically classified as a Seyfert 1.8 (Moran et al. 1996), although it was classified as a type 1 in 2014, when it was also classified as a changing-look AGN (Shappee et al. 2014). A radio counterpart was detected with the VLA (Condon et al. 1998).

This galaxy was observed twice with XMM-Newton in 2013. These data were studied by Giustini et al. (2017), who reported changes in flux, spectral shape $(\Delta \Gamma \sim 0.1)$ and in $N_{\mathrm{H}}$ within the month timescale. Our analysis of the same XMM-Newton data showed that SMF2 is the best representation of the data set, with changes in $\operatorname{Norm}_{1}(59 \%)$ and $N_{\mathrm{H} 2}(30 \%)$ within a one month period. This implies flux variations of $46 \%(45 \%)$ in the soft (hard) energy band. A follow up of this source of $\sim 70$ days using Xray, UV, optical and NIR data was conducted by Shappee et al. (2014) after a transient source alert on 2013 April. They observed an increasing X-ray flux of about one order of magnitude followed by an increase in its optical/UV continuum flux. By cross-correlating the light curves, they found that the UV (between two and three days) and the NIR (six to nine days) lagged behing the X-rays, and explained this variability behavior due to $\mathrm{X}$-ray radiation driven by the disk.

Giustini et al. (2017) analyzed the XMM-Newton light curves and reported modest variations in the $0.3-10 \mathrm{keV}$ energy band. They also reported hints of a soft band delay on timescales larger than $5 \mathrm{ks}$ between the soft and the hard energy bands. From the analysis of one of the XMM-Newton light curves, we found short-term variations in the soft and total energy bands. In the UV, we detected variations in the $U V W I(30 \sigma)$ filter with data from the OM.

\section{B.8. MARK 1218}

MARK 1218 (also named NGC 2622) was optically classified as a Seyfert 1.8 by Osterbrock \& Dahari (1983), but it changed to a Seyfert 1 spectrum a few years later (Smith et al. 2004) and has also been classified as a type 1.9 (Trippe et al. 2010). A radio counterpart was detected with VLA data at 6 and $20 \mathrm{~cm}$ (Ulvestad 1986).

This source was observed with XMM-Newton twice in 2005. Variability studies of this source were not found in the literature. Singh et al. (2011) performed a spectral fit of the obsID. 0302260201 using an absorbed power law with warm absorption, and obtained an X-ray luminosity of $\log L(2-$ $10 \mathrm{keV})=42.68$, in very good agreement with our estimation. We found that the best representation of the data is SMF1 with 
changes in Norm (63\%). This implies flux changes of $64 \%$ in both the soft and hard enery bands.

In the UV, variations were not detected with the UVM2 $(1 \sigma)$ filter from the OM.

\section{B.9. NGC 2992}

NGC 2992 is interacting with NGC 2993, which is located at 2.9'. It was classified as a Seyfert 1.9 by Ward et al. (1980) using optical data. A radio counterpart was detected with VLA data at $5 \mathrm{GHz}$ (Condon et al. 1982). In good agreement with our results, it is also a changing-look candidate (Gilli et al. 2000; Trippe et al. 2008).

Historically, X-ray flux variability by a factor of 20 was found in this source between different satellites (Shu et al. 2010, and references therein). It was observed four times with Chandra between 2003 and 2010, and ten times with XMM-Newton between 2003 and 2013. Shu et al. (2010) studied the XMMNewton data from 2003 and reported flux variability when comparing with Suzaku data from 2005. They attributed the changes to intrinsic variability of the source. Parker et al. (2015) used the nine XMM-Newton observations between 2003 and 2010 to study variability through principal component analysis. They reported variations in two components, one corresponding to intrinsic source variability, and a more ambigous one, corresponding either to changes in the power law, the column density, or the soft excess. From the analysis of the XMM-Newton data, the best representation is obtained with SMF2 varying $N_{\mathrm{H} 2}(5 \%)$ and $\mathrm{Norm}_{2}(21 \%)$ in a three years period. This results in a flux variation of $19 \%$ in the soft and hard energy bands.

The covering of this source with RXTE during one year (24 observations) was presented by Murphy et al. (2007), who reported flux variations by a factor of ten in timescales of days to weeks. They related these variations with changes in the inner accretion disk.

González-Martín \& Vaughan (2012) studied the nine XMMNewton observations between 2003 and 2010 and reported shortterm variations in three energy bands but not in all the observations, being more frequent in the total and hard energy bands than in the soft band. We analyzed five XMM-Newton observations and detected variations in the total and hard energy bands in a few observations, but none in the soft band.

At higher energies, a combined study with INTEGRAL, Swift, and BeppoSAX data published by Beckmann et al. (2007) showed that variations in the normalization of the power law were needed when using an absorbed broken power law model to fit the data simultaneously. They found a constant $\Gamma$ and flux variations by a factor of 11 in timescales of months to years.

Variations in the near infrared were reported by Glass (1997) due to an outburst between March 1988 and April 1992 observed with the $1.9 \mathrm{~m}$ telescope at Sutherland.

At UV frequencies, variations in the $U V M 2$ filter of $6 \sigma$ were detected with the OM.

\section{B.10. POX 52}

POX 52 is a dwarf elliptical galaxy. It was optically classified as a Seyfert 1.8 by Barth et al. (2004). A radio counterpart was observed with VLA data at $5 \mathrm{GHz}$ (Thornton et al. 2008).

This source was observed once by XMM-Newton in 2005 and once by Chandra in 2006. Thornton et al. (2008) studied these observations. They found variations in the column density due to partial covering during this period. This result agrees well with our analysis, where variations in $N_{\mathrm{H} 2}(44 \%)$ and $N_{\mathrm{H} 1}$ (it passes from a value of $\sim 8 \times 10^{22} \mathrm{~cm}^{-2}$ to the Galactic value) are needed to explain the observed variations in the same dataset. We note however that our values of $N_{\mathrm{H} 2}$ are larger than theirs, most probably due to the different models used (we used two absorbers instead of one). The annular region contributed with $4 \%$ to the Chandra data.

González-Martín et al. (2011) studied a sample of ultraluminous X-ray sources (ULX) using XMM-Newton data and included this source because the AGN has a low black hole mass. They reported short-term variations through the estimation of $\sigma_{\text {NXS }}^{2}$ in the 2-10 keV energy band. Dewangan et al. (2008) also estimated the $\sigma_{\text {NXS }}^{2}$ and found it to be compatible with short-term variations for the same XMM-Newton observation. The Chandra and $X M M-N e w t o n$ light curves were analyzed by Thornton et al. (2008), who found variations in timescales of $500 \mathrm{~s}$ and $10^{4} \mathrm{~s}$, respectively, in the $0.5-10 \mathrm{keV}$ energy band. These results agree well with our analysis of the XMM-Newton light curve, where we also detected these variations.

At UV frequencies, Thornton et al. (2008) studied the OM data and also GALEX data, but variations were not detected.

\section{B.11. NGC 4138}

NGC 4138 is a spiral galaxy that was classified as a Seyfert 1.9 by Ho et al. (1997). A radio counterpart was detected using VLA data at $2 \mathrm{~cm}$ (Nagar et al. 2002).

This galaxy was observed once with XMM-Newton in 2001 and once with Chandra in 2003. Variability studies of this source were not found in the literature. The XMM-Newton spectrum is best fitted with the ME2PL model, but the Chandra spectrum did not have enough counts below $\sim 2 \mathrm{KeV}$, and thus the PL model was used to fit both spectra individually and also for the simultaneous fit in the 2-10 keV energy band. The annular region contributed with $83 \%$ in Chandra data. SMF1 with variations in Norm (98\%) represents best the data in a two years period. This implies flux variations of $97 \%(21 \%)$ in the soft (hard) energy band.

\section{B.12. NGC 4395}

NGC 4395 is a late-type spiral galaxy that holds an intermediate mass black hole. Its nucleus was classified as a Seyfert 1.8 by Ho et al. (1997). A nuclear radio source was detected with VLBA data (Wrobel \& Ho 2006).

NGC 4395 was observed four times with Chandra between 2000 and 2004 and six times with XMM-Newton between 2002 and 2014. O'Neill et al. (2006) studied three Chandra spectra plus the XMM-Newton spectrum from November 2003. They reported flux variations of a factor of two between the Chandra and XMM-Newton observations. Nardini \& Risaliti (2011) studied the XMM-Newton data from November 2003 and Suzaku data obtained in June 2007. They reported variations related to the covering fraction of the neutral absorber and discussed that this absorber is located in the BLR. These studies agree well with our results. The best representation of the Chandra data shows variations in $N_{\mathrm{H} 2}(31 \%)$ within one day when fitting SMF1. XMMNewton data require SMF2 with $N_{\mathrm{H} 2}(20 \%)$ and Norm $_{2}(88 \%)$ varying in $12 \mathrm{yr}$ period. This implies flux variability of $15 \%$ $(13 \%)$ in the soft (hard) energy band. When comparing Chan$d r a$ and $X M M-N e w t o n$ data, the annular region contributes with $14 \%$ in Chandra data, and SMF1 is used with variations in $N_{\mathrm{H} 2}$ $(93 \%)$ in a two years period. 
Dewangan et al. (2008) studied two XMM-Newton light curves (2002 May and 2003 November) and reported short-term variations only in the 2003 data. González-Martín \& Vaughan (2012) analyzed the XMM-Newton observation from November 2003 and reported variations in the total, soft, and hard energy bands.

Vaughan et al. (2005) studied the 2003 XMM-Newton light curves together and found a high variability amplitude, with the fractional rms exceeding the unity. They found that this source follows the rms-flux relation usually observed in accreting black holes (e.g., Hernández-García et al. 2015). Moran et al. (2005) studied the Chandra data from June 2000 and reported shortterm variations from the analysis of the light curve, with changes of a factor of between two and three. They suggested that these changes are related to variations of the absorbing medium. O'Neill et al. (2006) found short-term variations during one of the Chandra light curves. One XMM-Newton light curve is analyzed in the present work, which shows variations in the soft, hard, and total energy bands.

Time-lags between optical or UV frequencies and X-rays have been studied by McHardy et al. (2016) (using XMMNewton, OM (UVWl filter) and optical data in the $g$-band), Cameron et al. (2012; using Swift data), and O'Neill et al. (2006; using Chandra and HST/STIS data) and found lags of 473 and $788 \mathrm{~s}, 400 \mathrm{~s}$, and a zero-lag correlation, respectively, relative to the X-rays.

Variations of this source have also been detected at nearinfrared frequencies in timescales shorter than a day by Minezaki et al. (2006). They observed the source in 2004 with the $2 \mathrm{~m}$ telescope at the Haleaka Observatories and found variations in he $J$ and $H$ bands correlated with the $V$ band, whereas variations in the $K$ band were not detected.

UV variations are detected in the $U V W 1(9 \sigma)$ filter using the available OM data.

\section{B.13. NGC 4565}

NGC 4565 is an edgewise spiral galaxy. It was classified as a Seyfert 1.9 in the optical (Ho et al. 1997). A compact radio core was detected using VLA data at $2 \mathrm{~cm}$ (Nagar et al. 2002). Ho \& Ulvestad (2001) suggested that the nucleus may be variable at radio frequencies since flux measurements fluctuated by a factor of two between their VLA data and previous FIRST measurements.

This source was observed once by XMM-Newton in 2001 and twice by Chandra in 2000 and 2003. Cappi et al. (2006) analyzed the XMM-Newton data. They fitted the spectrum with an absorbed power law model with $N_{\mathrm{H}}=0.12_{-}^{+} 0.04 \times 10^{22} \mathrm{~cm}^{-2}$, $\Gamma=1.8_{-}^{+} 0.2$, and $\log L(2-10 \mathrm{keV})=39.4$. They compared their spectral fit to the one reported by Terashima \& Wilson (2003) of the Chandra data from 2000, finding a good agreement between the spectral parameters and intrinsic luminosities. Our analysis agree well with their spectral fits. We find that SMF1 is the best representation of the data with changes in $N_{\mathrm{H}}(48 \%)$ in two years period. The annular region contributed with $17 \%$ to Chandra data.

We examined a Chandra light curve and detected possible variations in the soft energy band.

\section{B.14. MARK 883}

It was optically classified as Seyfert 1.9 by Osterbrock \& Dahari (1983), who stated that the broad component is "barely detected", and later classified as a Seyfert 2 (Trippe et al. 2010). A radio counterpart was detected at $6 \mathrm{~cm}$ with VLA data (Ulvestad 1986).

This nucleus was observed four times with XMM-Newton between 2006 and 2010. Variability studies of this source were not found in the literature. From our analysis we find that SMF1 represents the data best, showing changes in Norm $(28 \%)$ in a timescale of four years. This implies flux variations of $28 \%$ in both the soft and hard energy bands.

Data at UV frequencies with the OM are available in the three filters. Variations are detected in the $U V W 1(13 \sigma)$ and $U V W 2(5 \sigma)$ filters but not in the UVM2 $(1 \sigma)$ filter.

\section{B.15. IRAS 20051-1117}

IRAS 20051-1117 is a luminous spiral galaxy. The line ratios of this galaxy located it in the boundarie between a composite and a Seyfert galaxy (Moran et al. 1996). It shows a broad component, so it was classified as a Seyfert 1.9 using optical data (Georgantopoulos et al. 2004; Shi et al. 2010). A radio counterpart was detected with VLA data at $1.4 \mathrm{GHz}$ (Condon et al. 1998). This is one of the cases where a source is classified as a type 2 AGN at optical wavelenghts but shows no absorption at X-ray frequencies (Panessa \& Bassani 2002).

It was observed once with Chandra and twice with $X M M-$ Newton in 2002. Georgantopoulos et al. (2004) and Shi et al. (2010) studied the Chandra and one XMM-Newton observations and reported no variations between the two epochs, separated by only three weeks. They obtained a luminosity of $\log L(2-$ $10 \mathrm{keV})=42.60$, in perfect agreement with our estimated luminosity for the same $X M M-N e w t o n$ spectrum. Using the two $X M M-N e w t o n$ observations, we found that changes in $\mathrm{Norm}_{2}$ (29\%) are required in the SMF1 within half a year. This results in flux variations of $29 \%$ in both the soft and hard energy bands.

Georgantopoulos et al. (2004) did not find short-term variations in the Chandra nor the XMM-Newton light curves.

At UV frequencies variations were not detected with the $U V W 1(2 \sigma)$ filter.

\section{Appendix C: Images}

\section{C.1. Optical spectra, and X-ray, 2MASS and optical HST images}

In this appendix we present images at different wavelengths for each energy and the optical spectrum when available from NED. In X-rays we extracted Chandra data in four energy bands: 0.6$0.9 \mathrm{keV}$ (top left), 1.6-2.0 keV (top middle), 4.5-8.0 keV (top right), and $0.5-10.0 \mathrm{keV}$ (bottom left). The CSMOOTH task included in CIAO was used to adaptatively smooth the three images in the top panels (i.e., the images in the $0.5-10.0 \mathrm{keV}$ energy band are not smoothed), using a fast Fourier transform algorithm and a minimum and maximum significance level of the $\mathrm{S} / \mathrm{N}$ of three and four, respectively. When data from Chandra was not available, XMM-Newton images were extracted in the same energy bands, and the ASMOOTH task was used to adaptatively smooth the images. At infrared frequencies, we retrieved an image from $2 \mathrm{MASS}$ in the $K_{\mathrm{s}}$ filter ${ }^{10}$. At optical frequencies we used images from the Hubble Space telescope (HST) ${ }^{11}$, preferably in the $F 814 \mathrm{~W}$ filter, but when it was not available we

\footnotetext{
10 http://irsa.ipac.caltech.edu/applications/2MASS/IM/ interactive.html

11 http://hla.stsci.edu/
} 


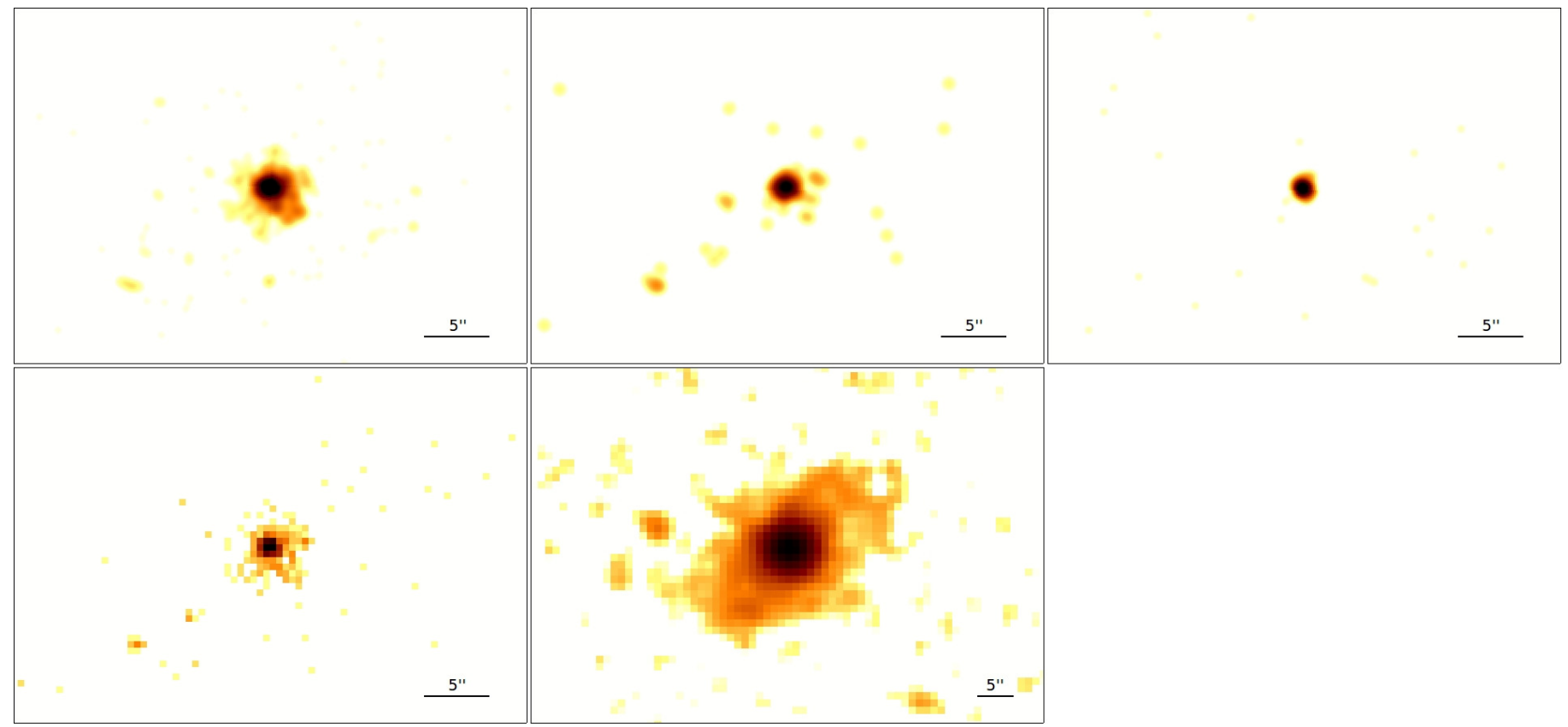

Fig. C.1. ESO 540-G01. Top left: smoothed X-ray 0.6-0.9 keV energy band; top center: smoothed X-ray 1.6-2.0 keV energy band; top right: smoothed X-ray 4.5-8.0 keV energy band; bottom left: X-ray 0.5-10.0 keV energy band without smoothing; bottom center: 2MASS image in the $K_{\mathrm{s}}$ band.

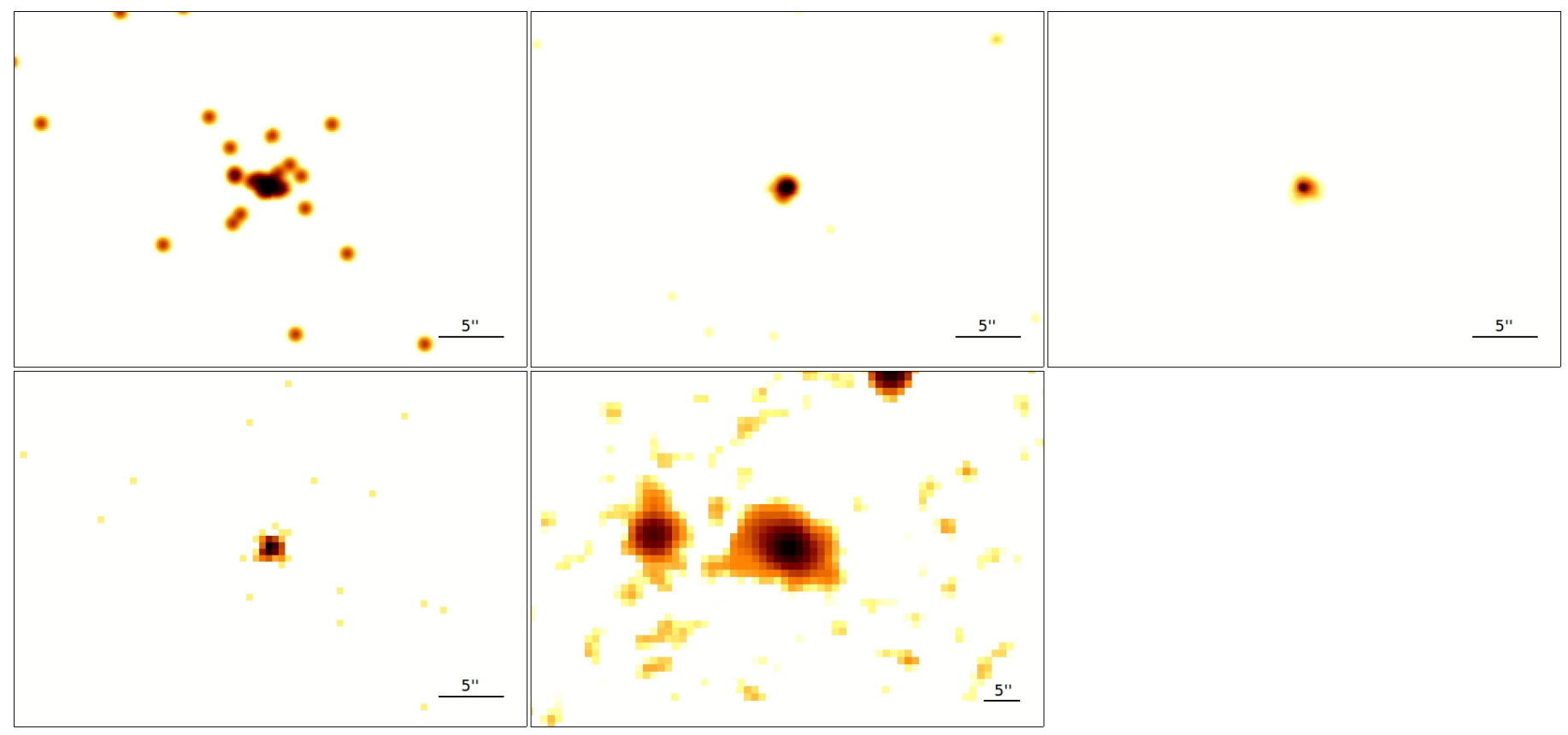

Fig. C.2. ESO 195-IG21. Top left: smoothed X-ray 0.6-0.9 keV energy band; top center: smoothed X-ray 1.6-2.0 keV energy band; top right: smoothed X-ray 4.5-8.0 keV energy band; bottom left: X-ray 0.5-10.0 keV energy band without smoothing; bottom center: 2MASS image in the $K_{\mathrm{s}}$ band.

retrieved an image in the $F 606 \mathrm{~W}$ filter. HST data have been processed following the sharp dividing method to show the internal structure of the galaxies (Marquez \& Moles 1996). The red squares in the bottom images represent the area covered by the HST image (presented in the bottom right panel when available). In all images the gray levels extend from twice the value of the background dispersion to the maximum value at the center of each galaxy. We used IRAF $^{12}$ to estimate these values.

\footnotetext{
$\overline{12 \text { http://iraf.noao.edu/ }}$
} 
L. Hernández-García et al.: X-ray variability in Seyfert 1.8/1.9 galaxies

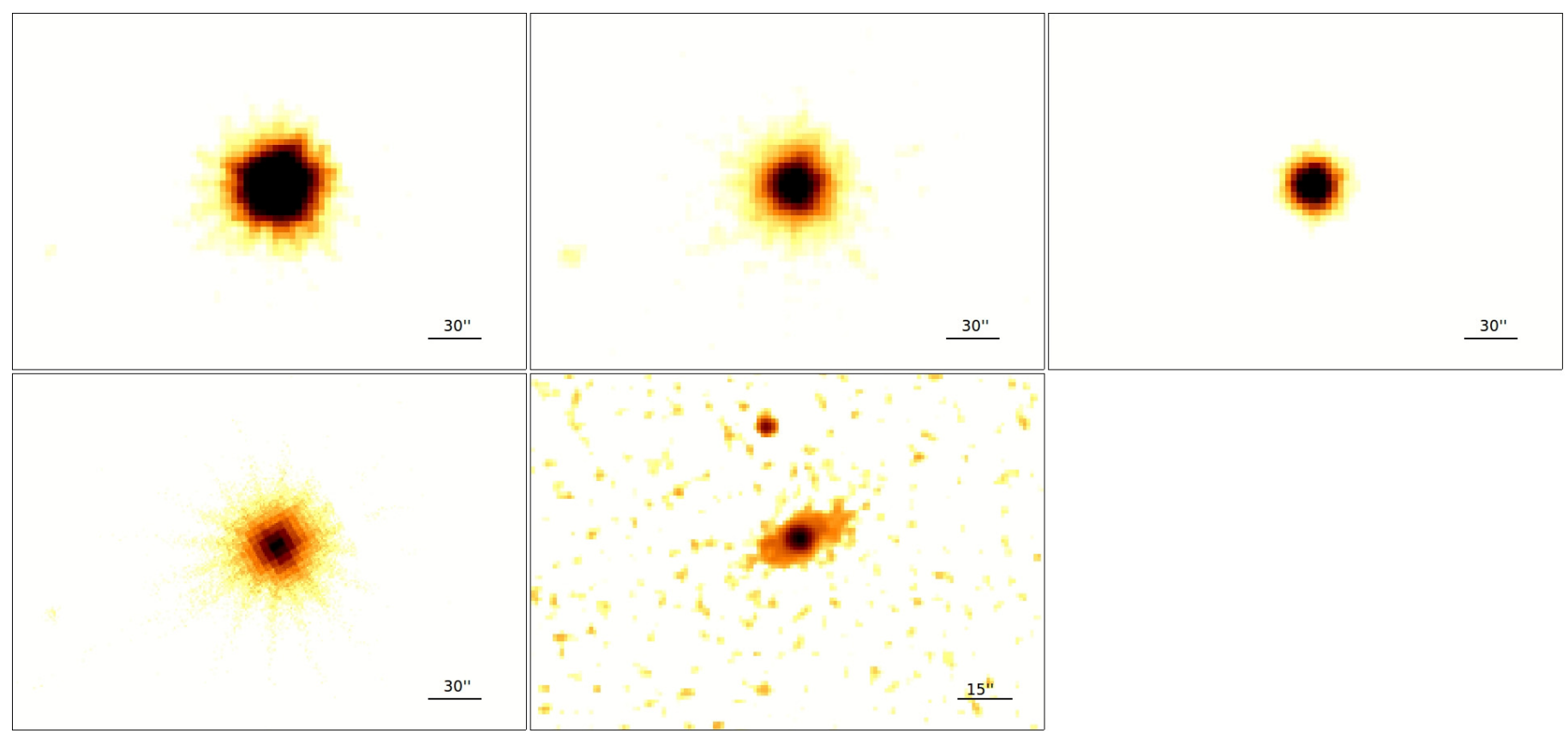

Fig. C.3. ESO 113-G10. Top left: smoothed X-ray 0.6-0.9 keV energy band; top center: smoothed X-ray 1.6-2.0 keV energy band; top right: smoothed X-ray 4.5-8.0 keV energy band; bottom left: X-ray 0.5-10.0 keV energy band without smoothing; bottom center: 2MASS image in the $K_{\mathrm{s}}$ band. 

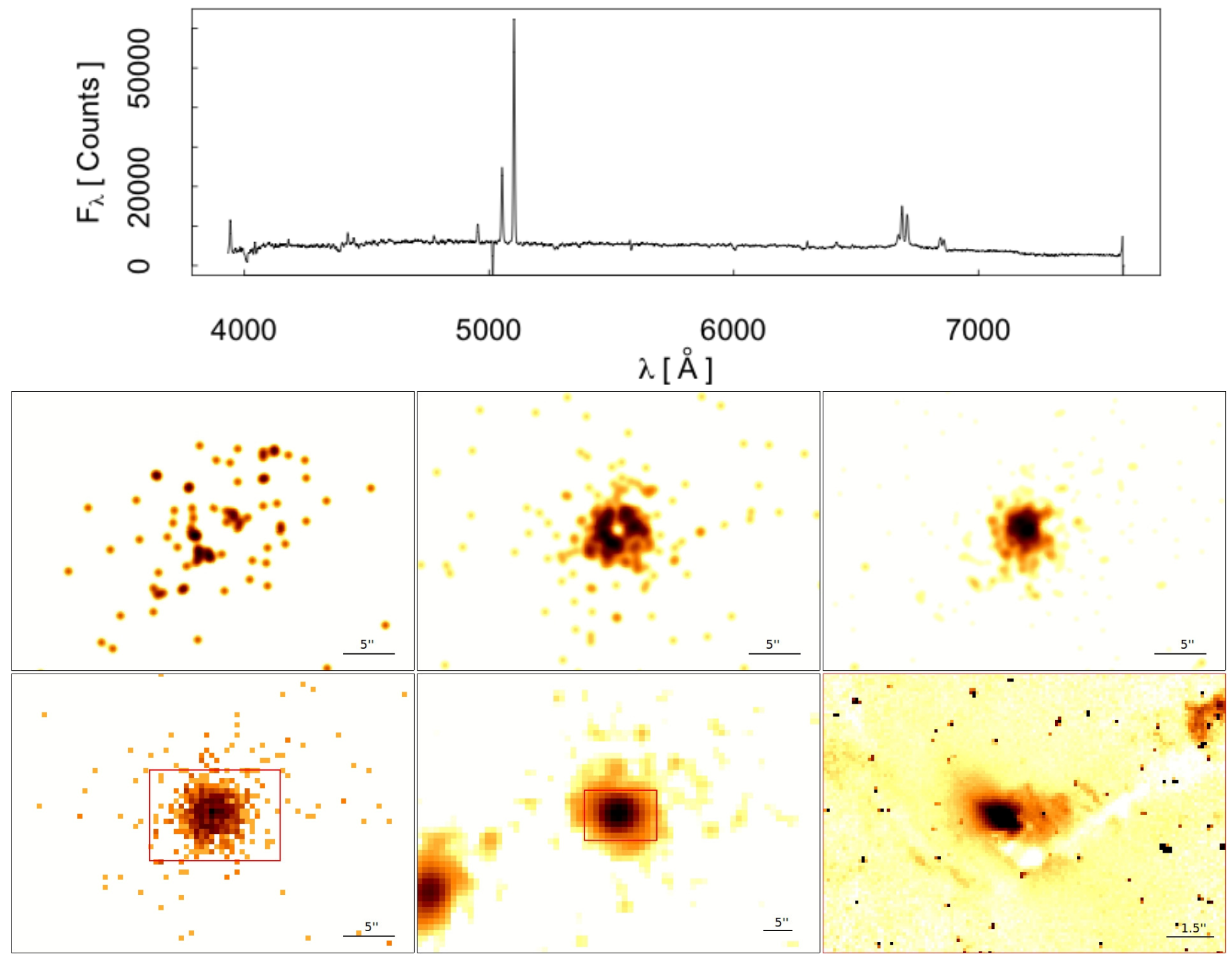

Fig. C.4. Top: optical spectrum (from NED); bottom: NGC 526A. Top left: smoothed X-ray 0.6-0.9 keV energy band; top center: smoothed X-ray 1.6-2.0 keV energy band; top right: smoothed X-ray 4.5-8.0 keV energy band; bottom left: X-ray 0.5-10.0 keV energy band without smoothing; bottom center: $2 \mathrm{MASS}$ image in the $K_{\mathrm{s}}$ band; bottom right: sharp divided Hubble image in the $F 606 W$ filter. 
L. Hernández-García et al.: X-ray variability in Seyfert 1.8/1.9 galaxies
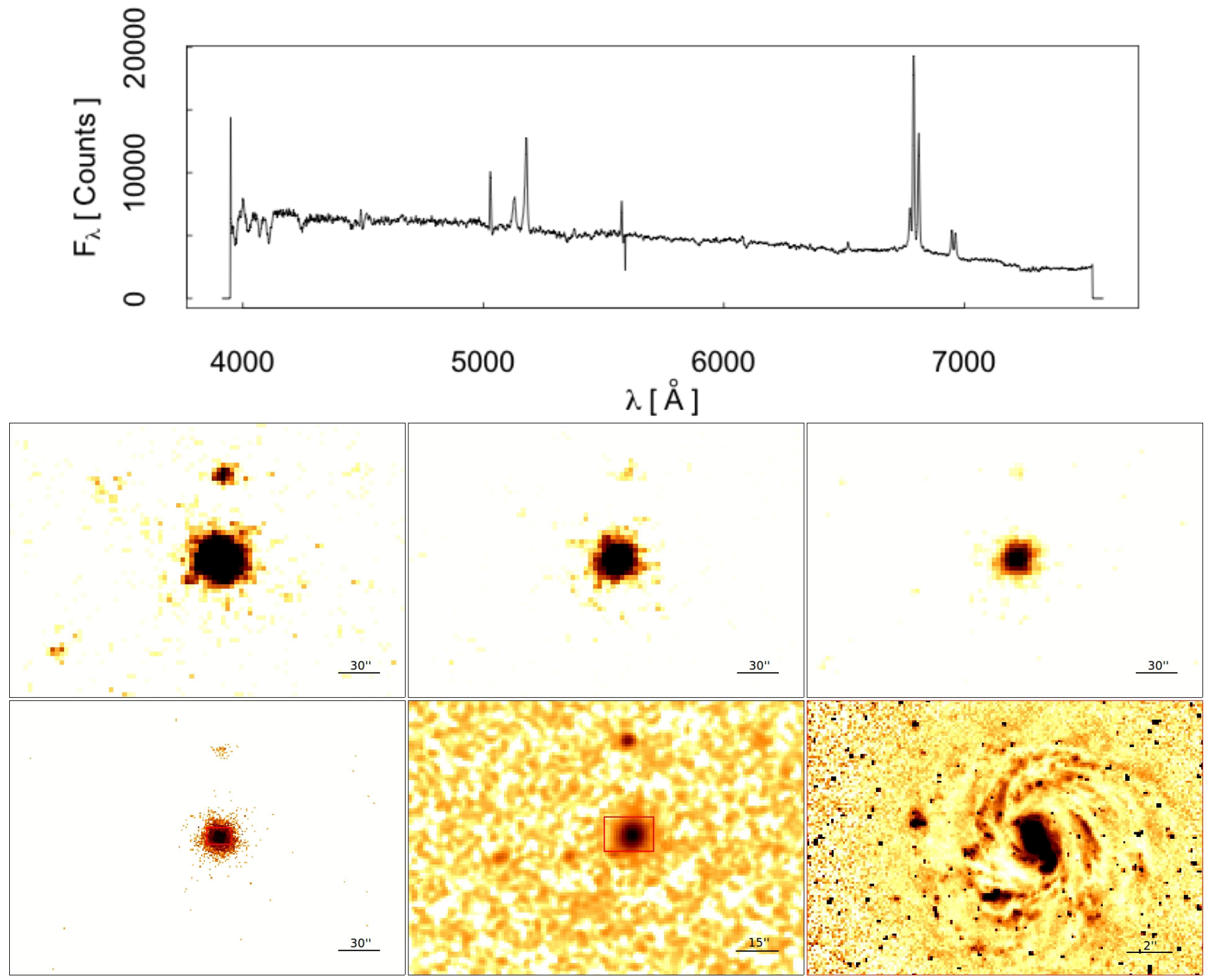

Fig. C.5. Top: optical spectrum (from NED); bottom: MARK 609. Top left: smoothed X-ray 0.6-0.9 keV energy band; top center: smoothed X-ray 1.6-2.0 keV energy band; top right: smoothed X-ray 4.5-8.0 keV energy band; bottom left: X-ray $0.5-10.0 \mathrm{keV}$ energy band without smoothing; bottom center: 2 MASS image in the $K_{\mathrm{s}}$ band; bottom right: Sharp divided Hubble image in the $F 606 W$ filter. 
A\&A 602, A65 (2017)
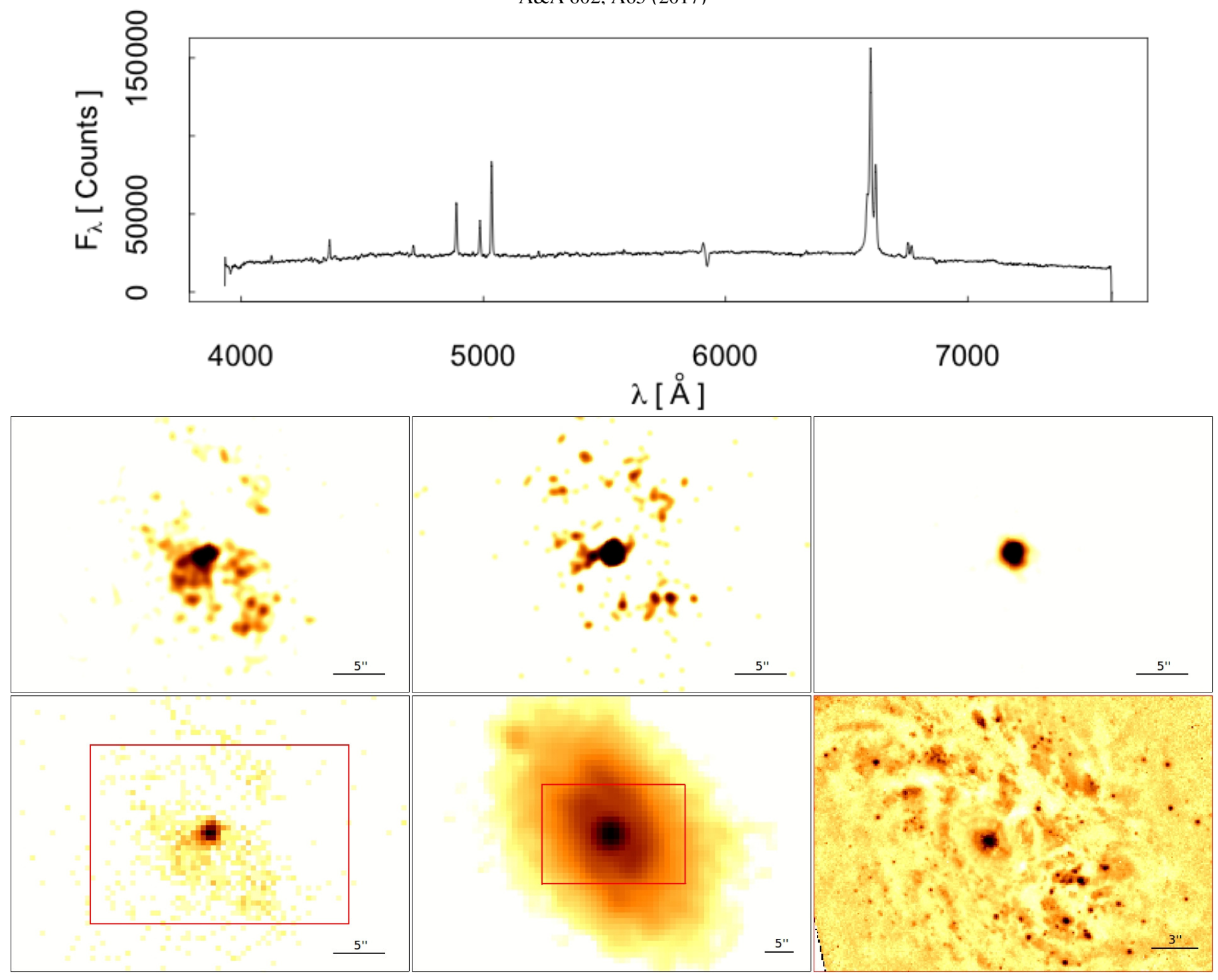

Fig. C.6. Top: optical spectrum (from NED); bottom: NGC 1365. Top left: smoothed X-ray 0.6-0.9 keV energy band; top center: smoothed X-ray 1.6-2.0 keV energy band; top right: smoothed X-ray 4.5-8.0 keV energy band; bottom left: X-ray 0.5-10.0 keV energy band without smoothing; bottom center: 2 MASS image in the $K_{\mathrm{s}}$ band; bottom right: sharp divided Hubble image in the $F 814 W$ filter. 
L. Hernández-García et al.: X-ray variability in Seyfert 1.8/1.9 galaxies
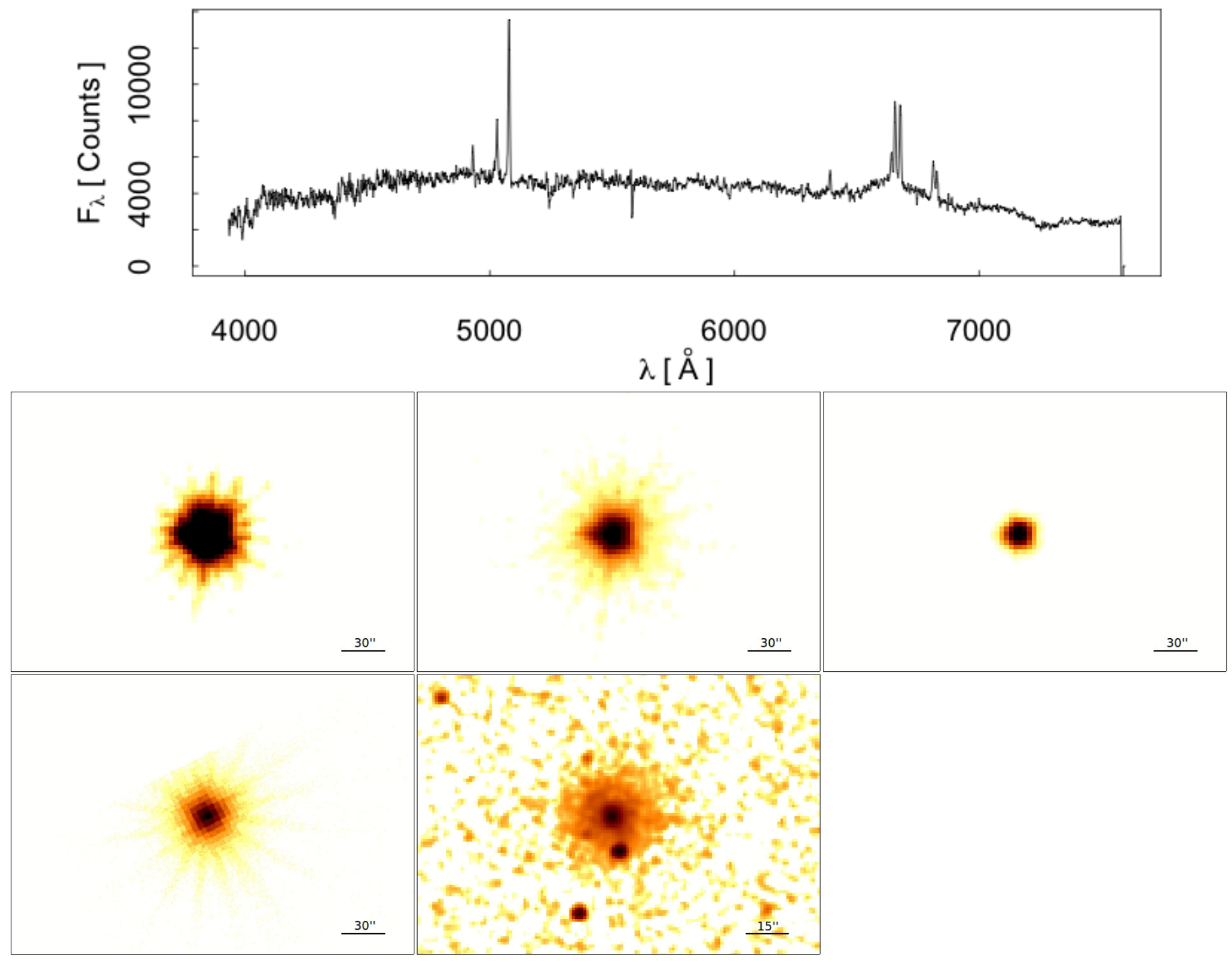

Fig. C.7. Top: optical spectrum (from NED); bottom: NGC 2617. Top left: smoothed X-ray 0.6-0.9 keV energy band; top center: smoothed X-ray 1.6-2.0 keV energy band; top right: smoothed X-ray 4.5-8.0 keV energy band; bottom left: X-ray 0.5-10.0 keV energy band without smoothing; bottom center: 2 MASS image in the $K_{\mathrm{s}}$ band. 
A\&A 602, A65 (2017)

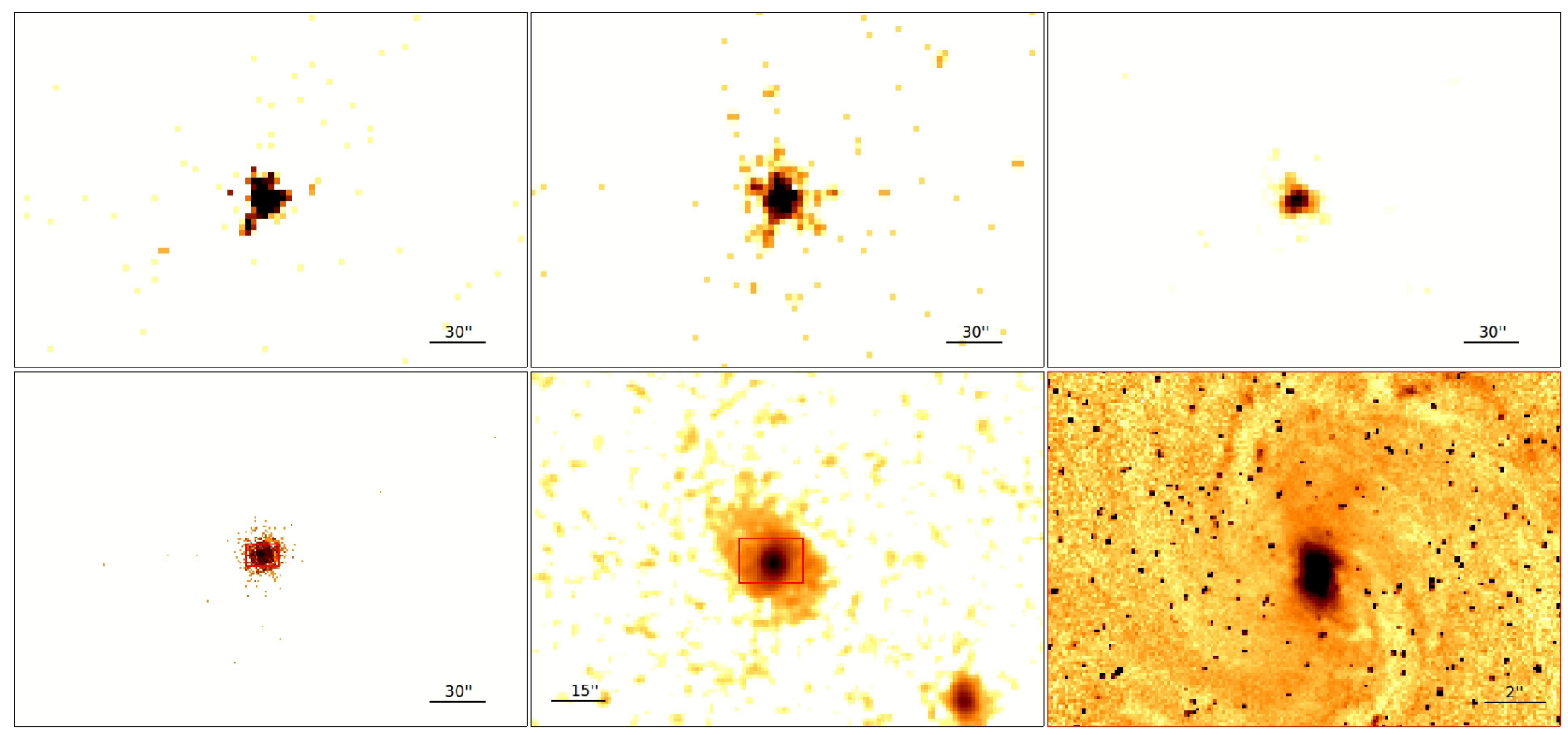

Fig. C.8. MARK 1218. Top left: smoothed X-ray 0.6-0.9 keV energy band; top center: smoothed X-ray 1.6-2.0 keV energy band; top right: smoothed X-ray 4.5-8.0 keV energy band; bottom left: X-ray 0.5-10.0 keV energy band without smoothing; bottom center: 2 MASS image in the $K_{\mathrm{s}}$ band; bottom right: sharp divided Hubble image in the $F 606 \mathrm{~W}$ filter. 
L. Hernández-García et al.: X-ray variability in Seyfert 1.8/1.9 galaxies
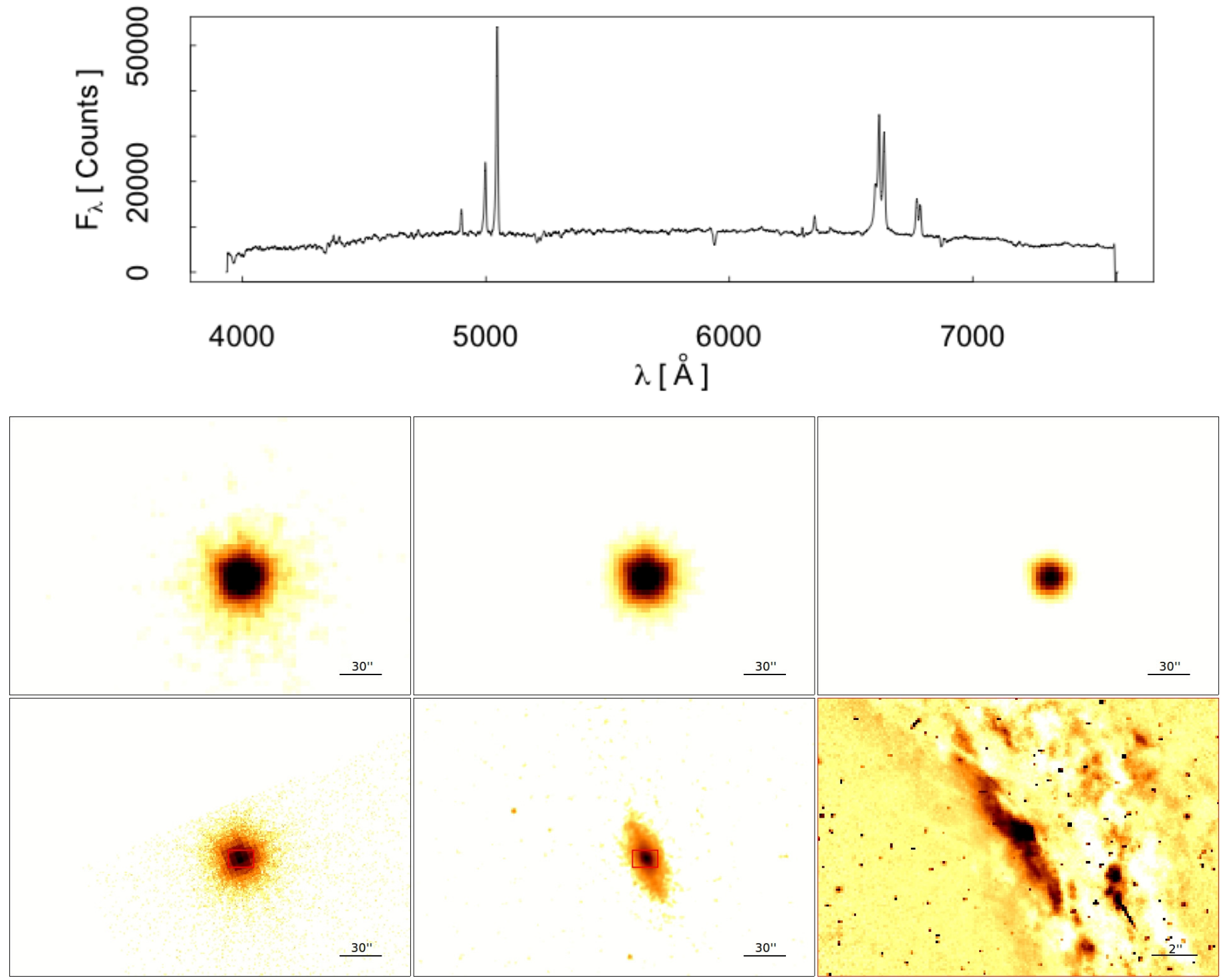

Fig. C.9. Top: optical spectrum (from NED); bottom: NGC 2992. Top left: smoothed X-ray 0.6-0.9 keV energy band; top center: smoothed X-ray 1.6-2.0 keV energy band; top right: smoothed X-ray 4.5-8.0 keV energy band; bottom left: X-ray 0.5-10.0 keV energy band without smoothing; bottom center: $2 \mathrm{MASS}$ image in the $K_{\mathrm{s}}$ band; bottom right: sharp divided Hubble image in the $F 606 W$ filter. 
A\&A 602, A65 (2017)

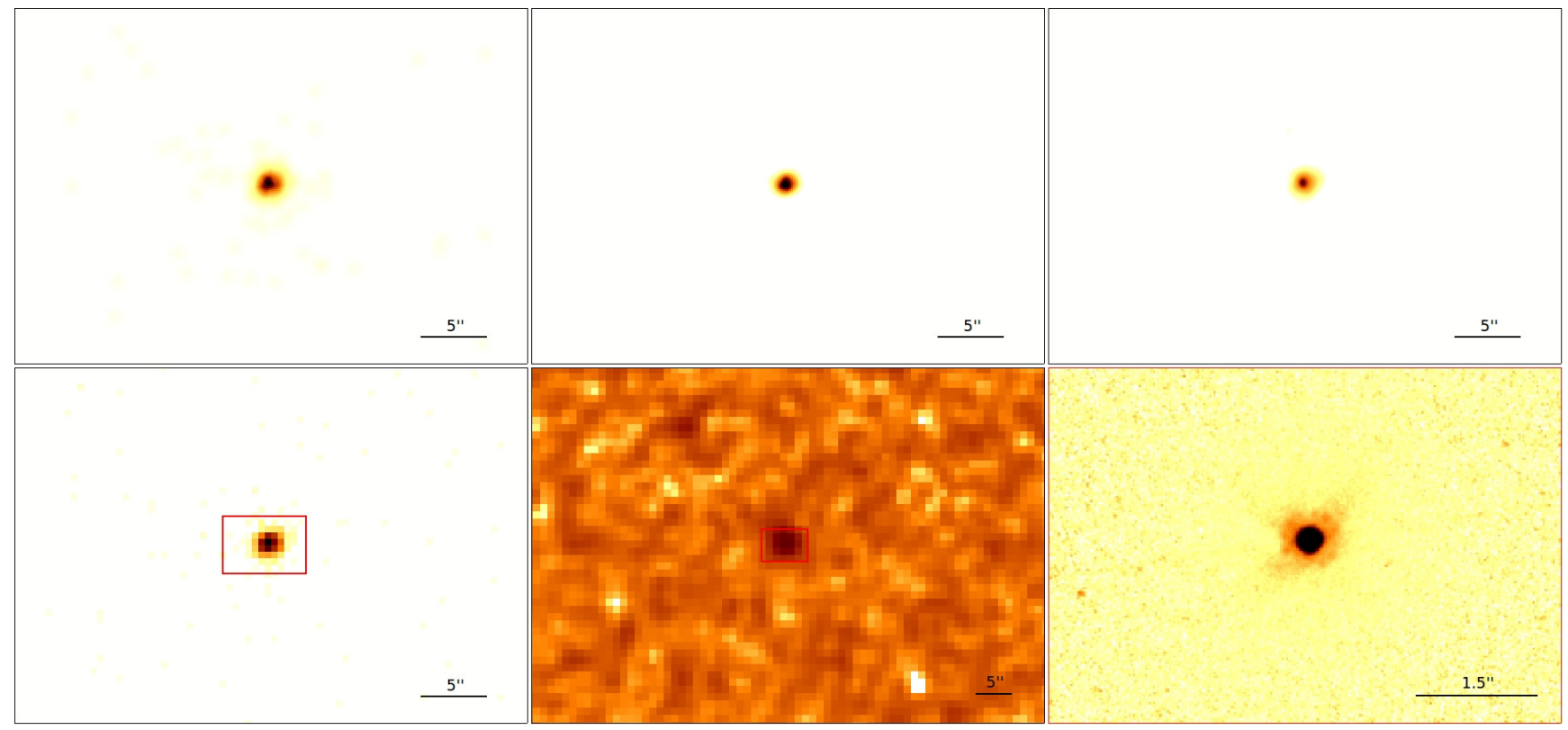

Fig. C.10. POX 52. Top left: smoothed X-ray 0.6-0.9 keV energy band; top center: smoothed X-ray 1.6-2.0 keV energy band; top right: smoothed X-ray $4.5-8.0 \mathrm{keV}$ energy band; bottom left: X-ray $0.5-10.0 \mathrm{keV}$ energy band without smoothing; bottom center: $2 \mathrm{MASS}$ image in the $K_{\mathrm{s}}$ band; bottom right: sharp divided Hubble image in the $F 814 W$ filter. 
L. Hernández-García et al.: X-ray variability in Seyfert 1.8/1.9 galaxies
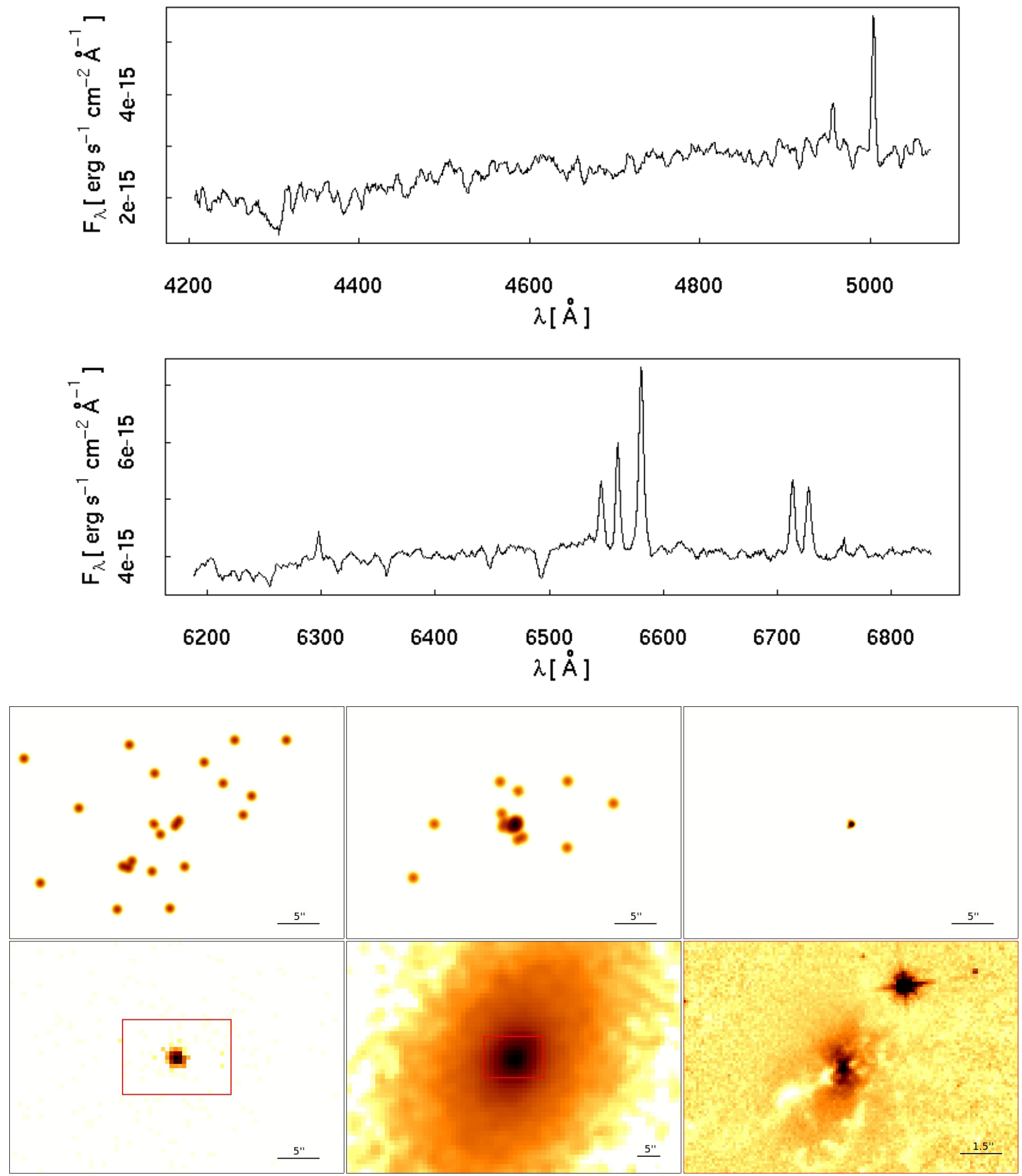

Fig. C.11. Top: optical spectra (from NED); bottom: NGC 4138. Top left: smoothed X-ray 0.6-0.9 keV energy band; top center: smoothed X-ray $1.6-2.0 \mathrm{keV}$ energy band; top right: smoothed X-ray $4.5-8.0 \mathrm{keV}$ energy band; bottom left: X-ray $0.5-10.0 \mathrm{keV}$ energy band without smoothing; bottom center: $2 \mathrm{MASS}$ image in the $K_{\mathrm{s}}$ band; bottom right: sharp divided Hubble image in the $F 547 W$ filter. 
A\&A 602, A65 (2017)
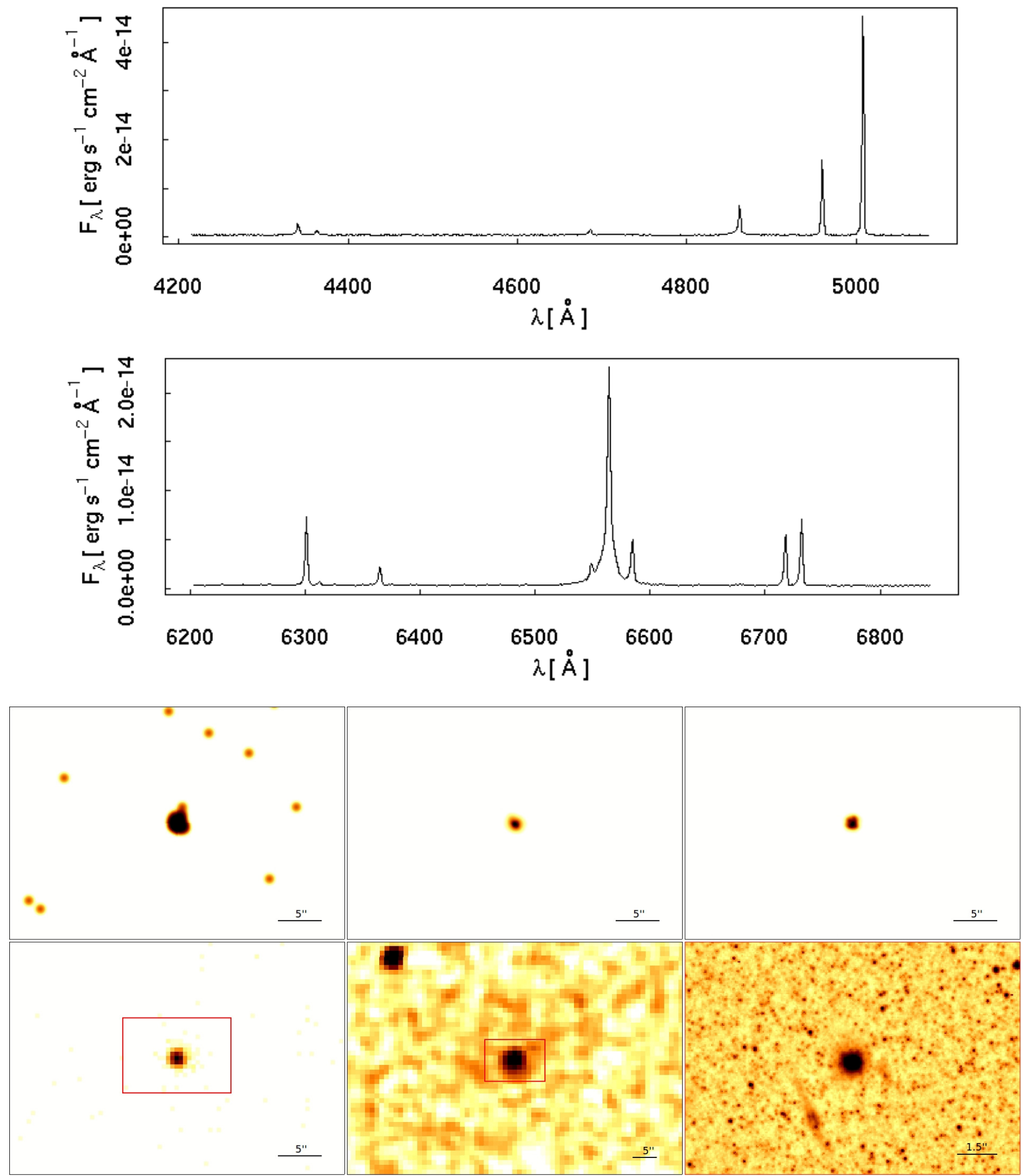

Fig. C.12. Top: optical spectra (from NED); bottom: NGC 4395. Top left: smoothed X-ray 0.6-0.9 keV energy band; top center: smoothed X-ray 1.6-2.0 keV energy band; top right: smoothed X-ray 4.5-8.0 keV energy band; bottom left: X-ray $0.5-10.0 \mathrm{keV}$ energy band without smoothing; bottom center: $2 \mathrm{MASS}$ image in the $K_{\mathrm{s}}$ band; (bottom right): sharp divided Hubble image in the $F 814 W$ filter. 
L. Hernández-García et al.: X-ray variability in Seyfert 1.8/1.9 galaxies
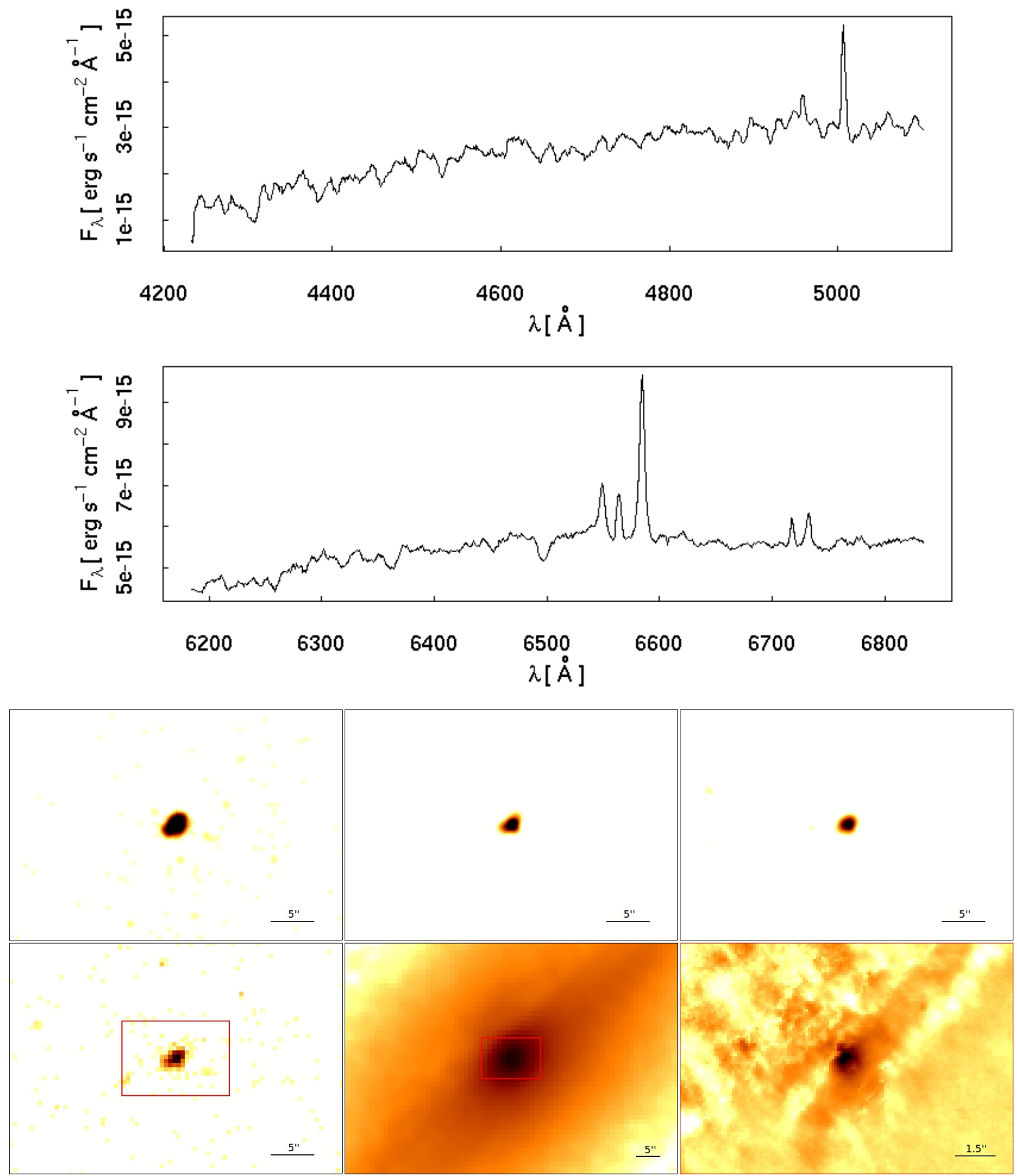

Fig. C.13. Top: optical spectra (from NED); bottom: NGC 4565. Top left: smoothed X-ray 0.6-0.9 keV energy band; top center: smoothed X-ray 1.6-2.0 keV energy band; top right: smoothed X-ray 4.5-8.0 keV energy band; bottom left: X-ray 0.5-10.0 keV energy band without smoothing; bottom center: 2 MASS image in the $K_{\mathrm{s}}$ band; bottom right: Sharp divided Hubble image in the $F 814 W$ filter. 
A\&A 602, A65 (2017)

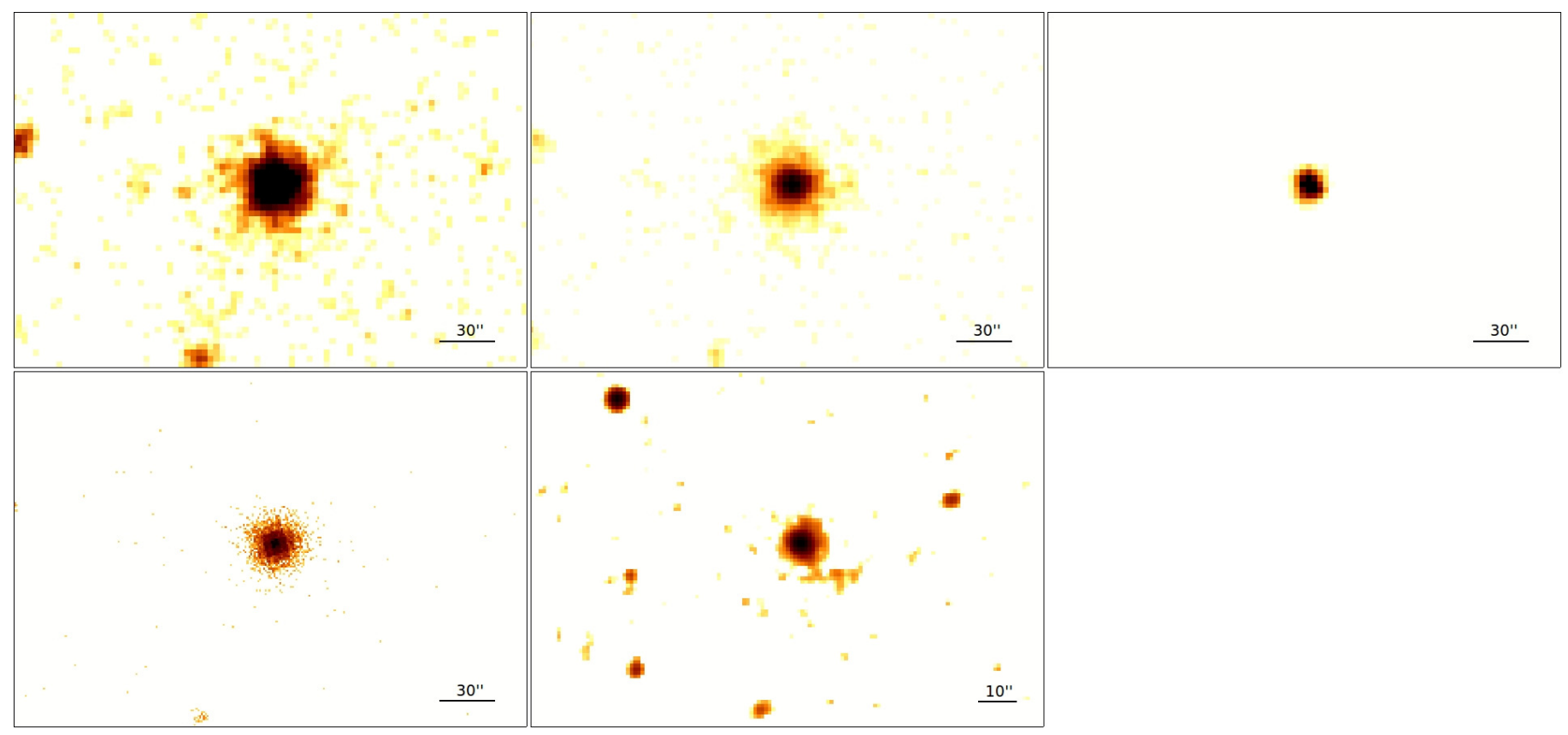

Fig. C.14. MARK 883. Top left: Smoothed X-ray 0.6-0.9 keV energy band; top center: smoothed X-ray 1.6-2.0 keV energy band; top right: smoothed X-ray 4.5-8.0 keV energy band; bottom left: X-ray 0.5-10.0 keV energy band without smoothing; bottom center: 2 MASS image in the $K_{\mathrm{s}}$ band. 
L. Hernández-García et al.: X-ray variability in Seyfert 1.8/1.9 galaxies
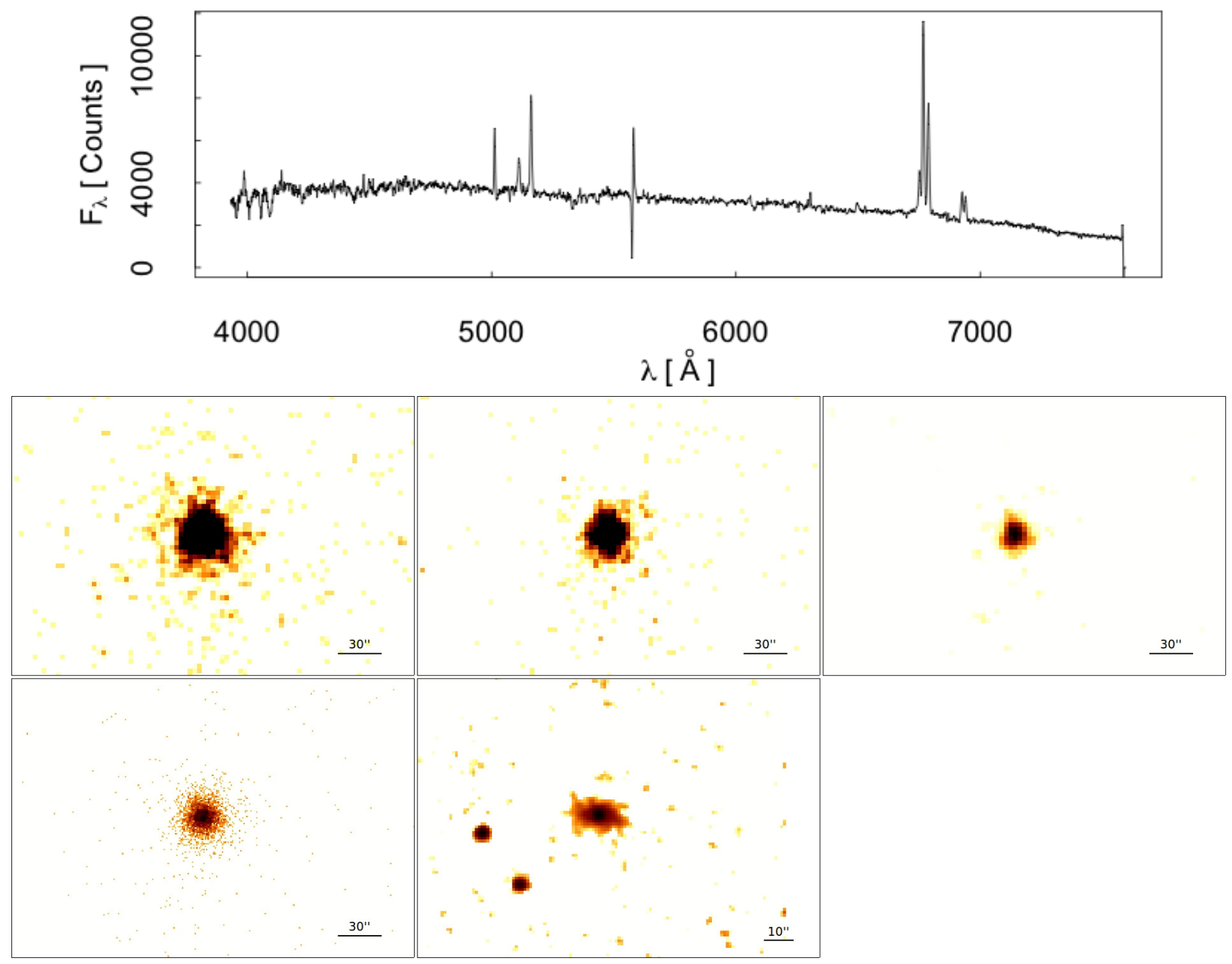

Fig. C.15. Top: optical spectrum (from NED); bottom: IRAS 20051-1117. Top left: smoothed X-ray 0.6-0.9 keV energy band; top center: smoothed X-ray 1.6-2.0 keV energy band; top right: smoothed X-ray 4.5-8.0 keV energy band; bottom left: X-ray 0.5-10.0 keV energy band without smoothing; bottom center: 2 MASS image in the $K_{\mathrm{s}}$ band.

\section{C.2. Chandra and XMM-Newton images}

In this appendix we present the images from Chandra (left) and XMM-Newton (right) that were used to compare the spectra from these two instruments in the $0.5-10 \mathrm{keV}$ band. In all cases, the gray scales extend from twice the value of the background dispersion to the maximum value at the center of each galaxy. 


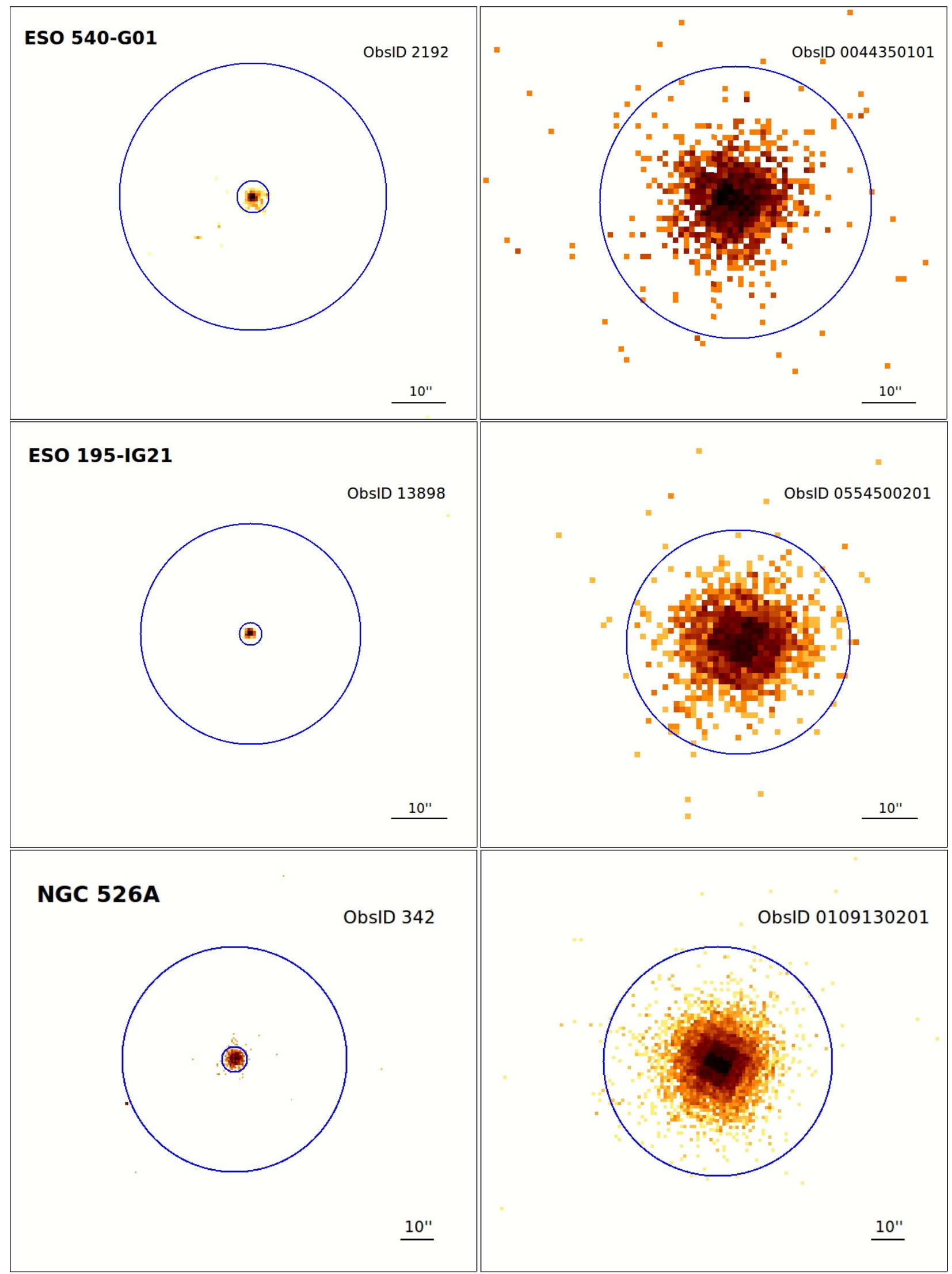

Fig. C.16. Chandra data (left) and XMM-Newton data (right) for the sources in the $0.5-10 \mathrm{keV}$ band. Big circles represent XMM-Newton data apertures. Small circles in the figures to the left represent the nuclear extraction aperture used with Chandra observations (see Table A.1). 
L. Hernández-García et al.: X-ray variability in Seyfert 1.8/1.9 galaxies

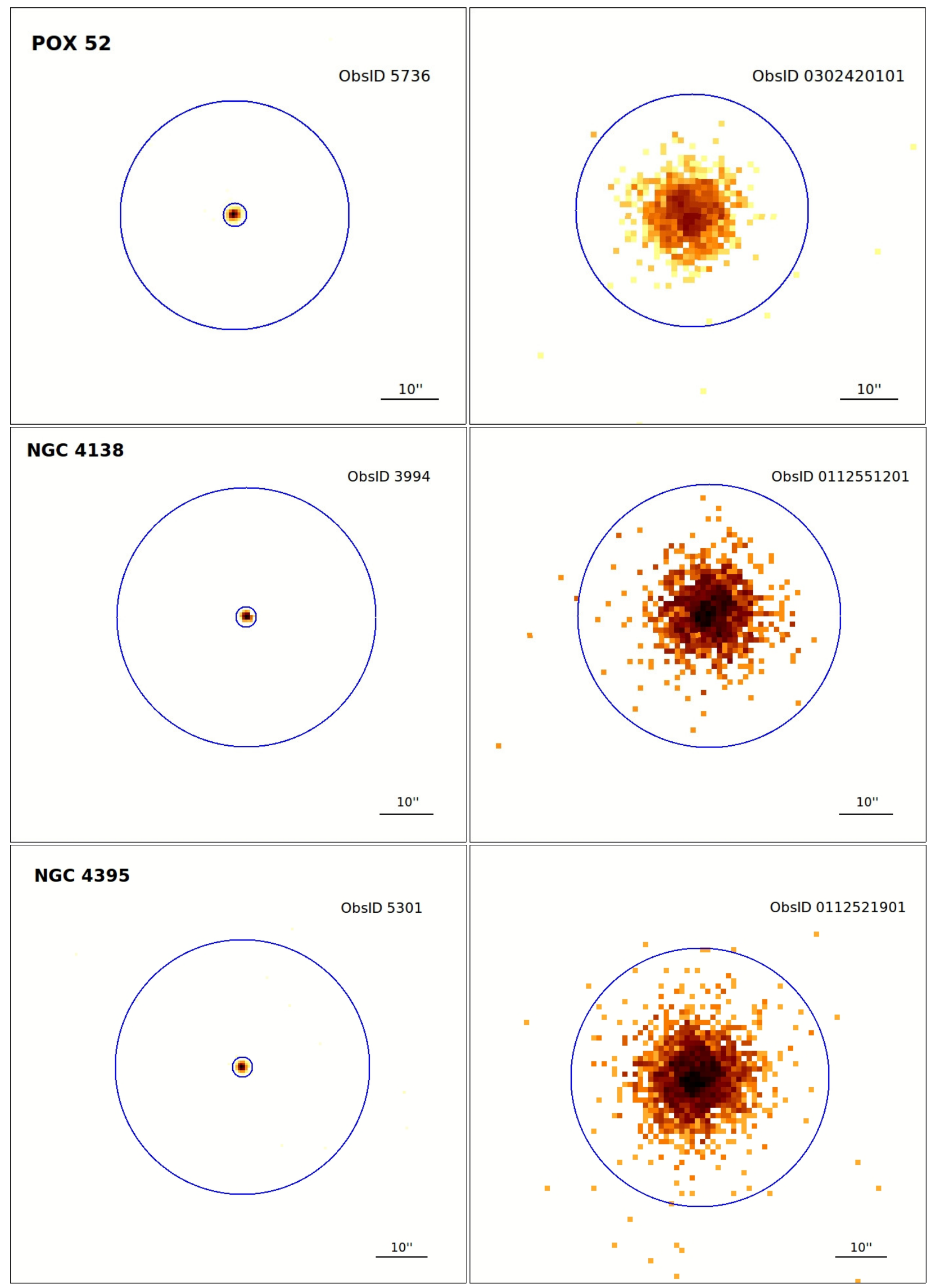

Fig. C.16. continued. 
A\&A 602, A65 (2017)

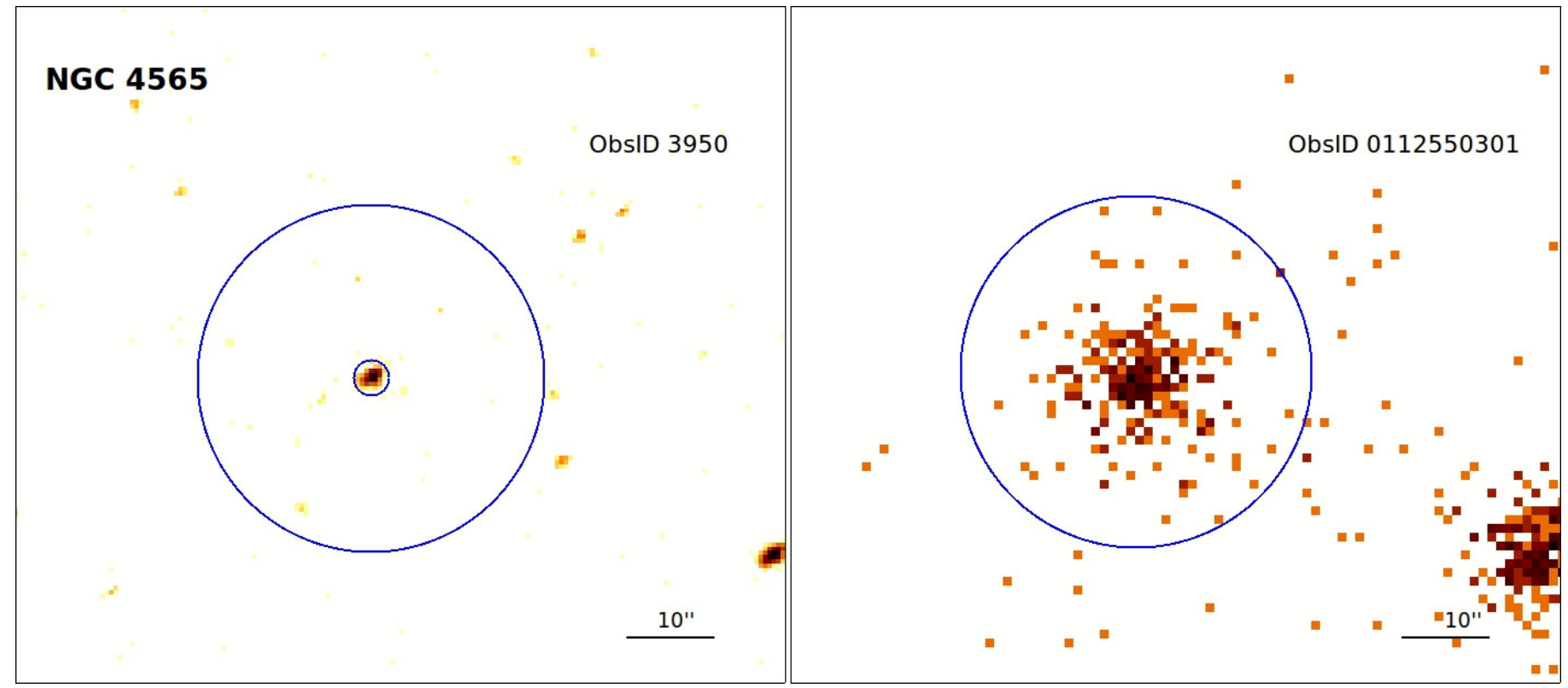

Fig. C.16. continued. 


\section{Appendix D: Light curves}

This appendix provides the plots corresponding to the light curves. Three plots per observation are presented, corresponding to soft (left), hard (middle), and total (right) energy bands. Each light curve has a minimum of $30 \mathrm{ks}$ (i.e., eight hours) exposure time, while long light curves are divided into segments of $40 \mathrm{ks}$ (i.e., $11 \mathrm{~h}$ ). Each segment is enumerated in the title of the light curve. Count rates versus time continua are represented. The solid line represents the mean value, dashed lines the ${ }_{-}^{+} 1 \sigma$ from the average.
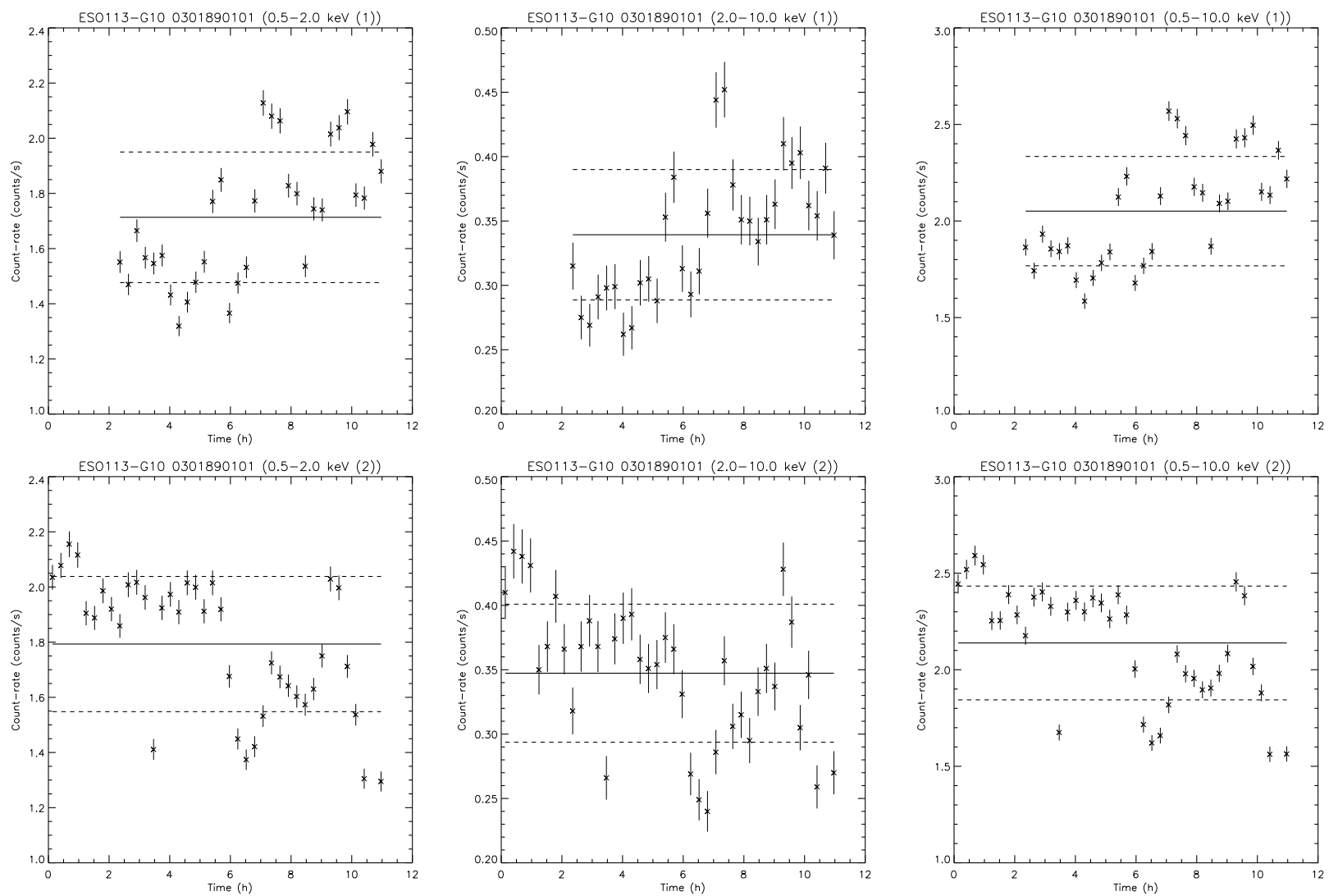

Fig. D.1. Light curves of ESO 113-G10 from XMM-Newton data. 
A\&A 602, A65 (2017)
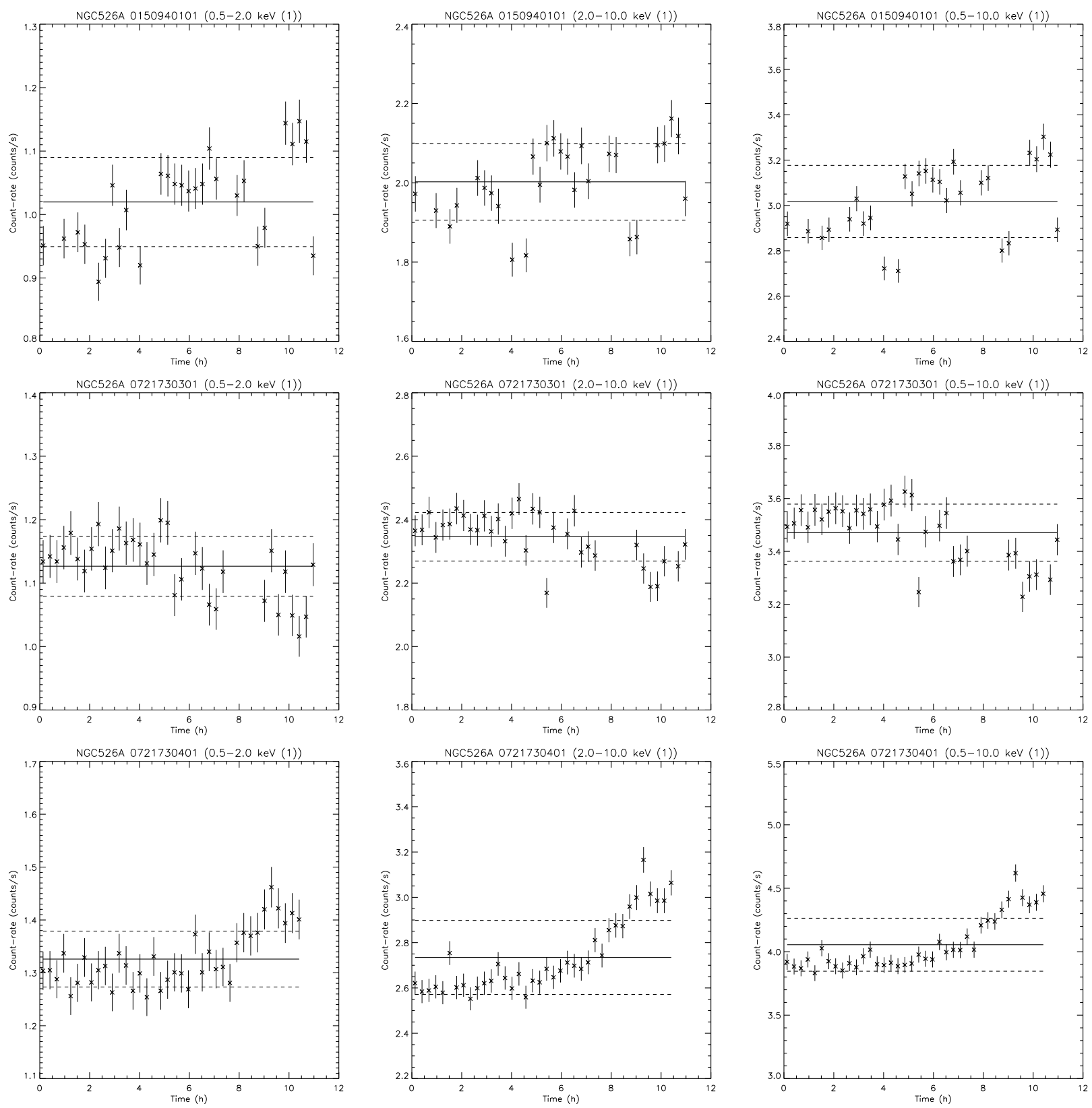

Fig. D.2. Light curves of NGC 526A from XMM-Newton data. 
L. Hernández-García et al.: X-ray variability in Seyfert 1.8/1.9 galaxies
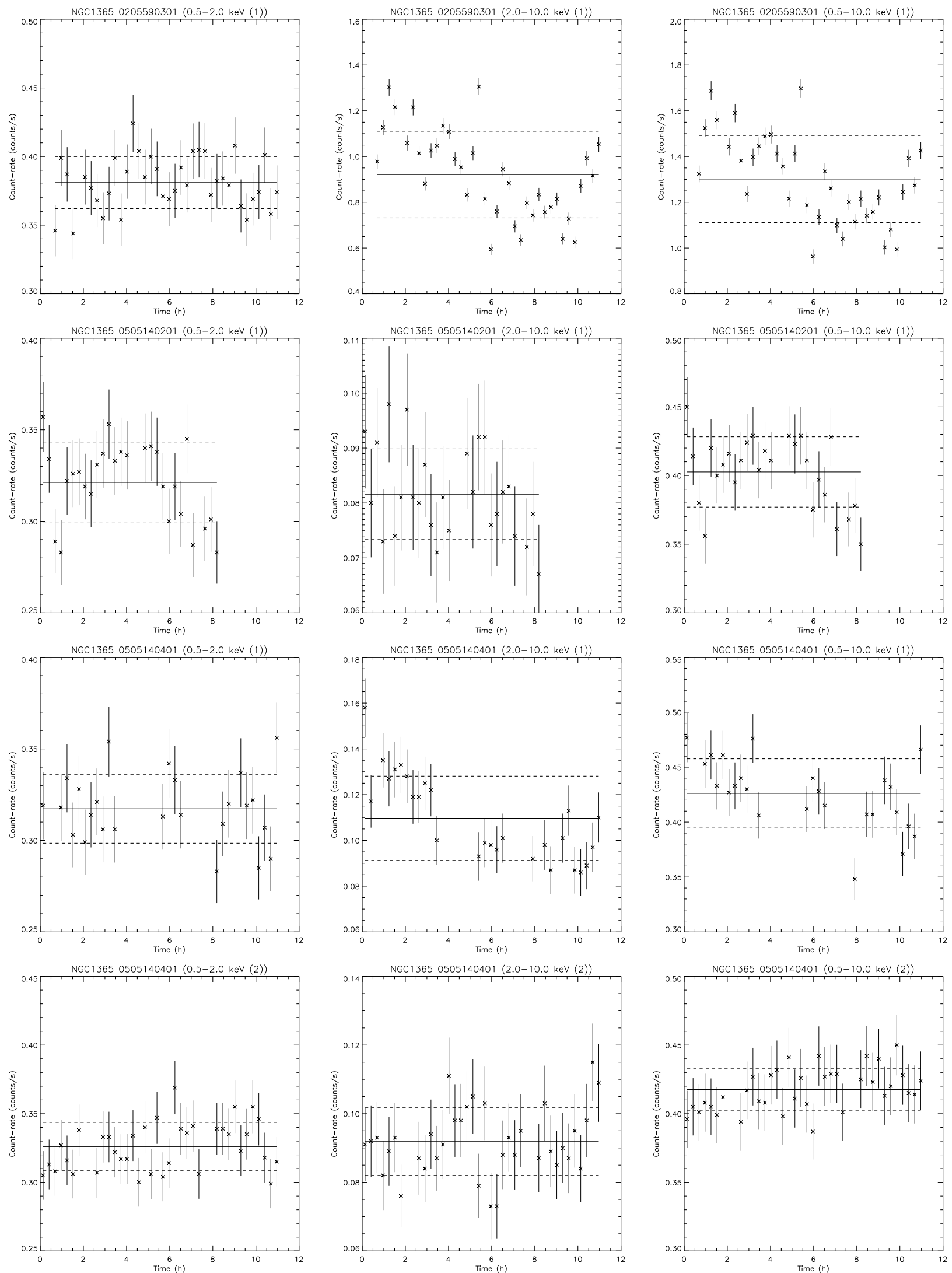

Fig. D.3. Light curves of NGC 1365 from XMM-Newton data. 
A\&A 602, A65 (2017)
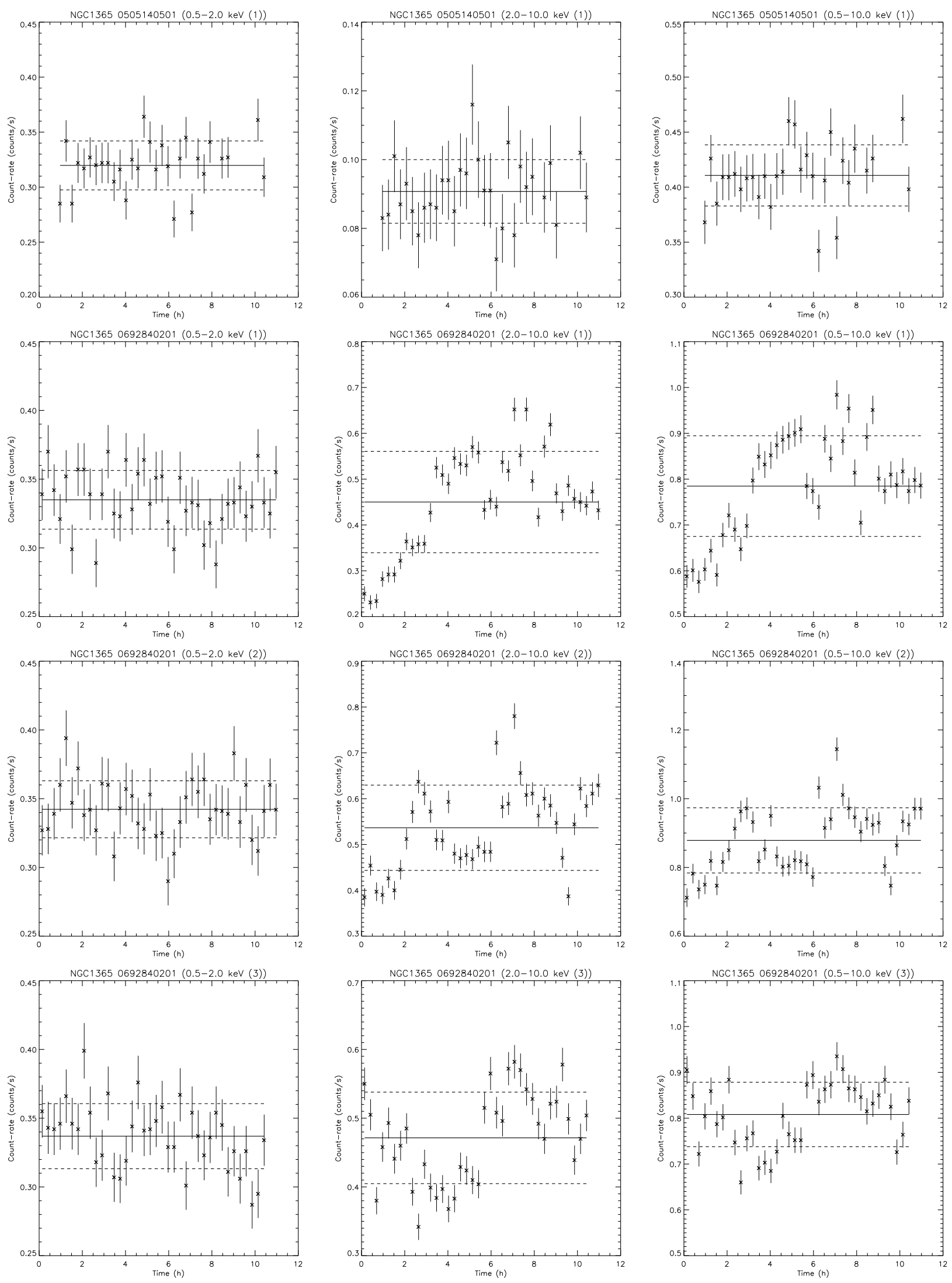

Fig. D.3. continued. 


\section{Hernández-García et al.: X-ray variability in Seyfert 1.8/1.9 galaxies}
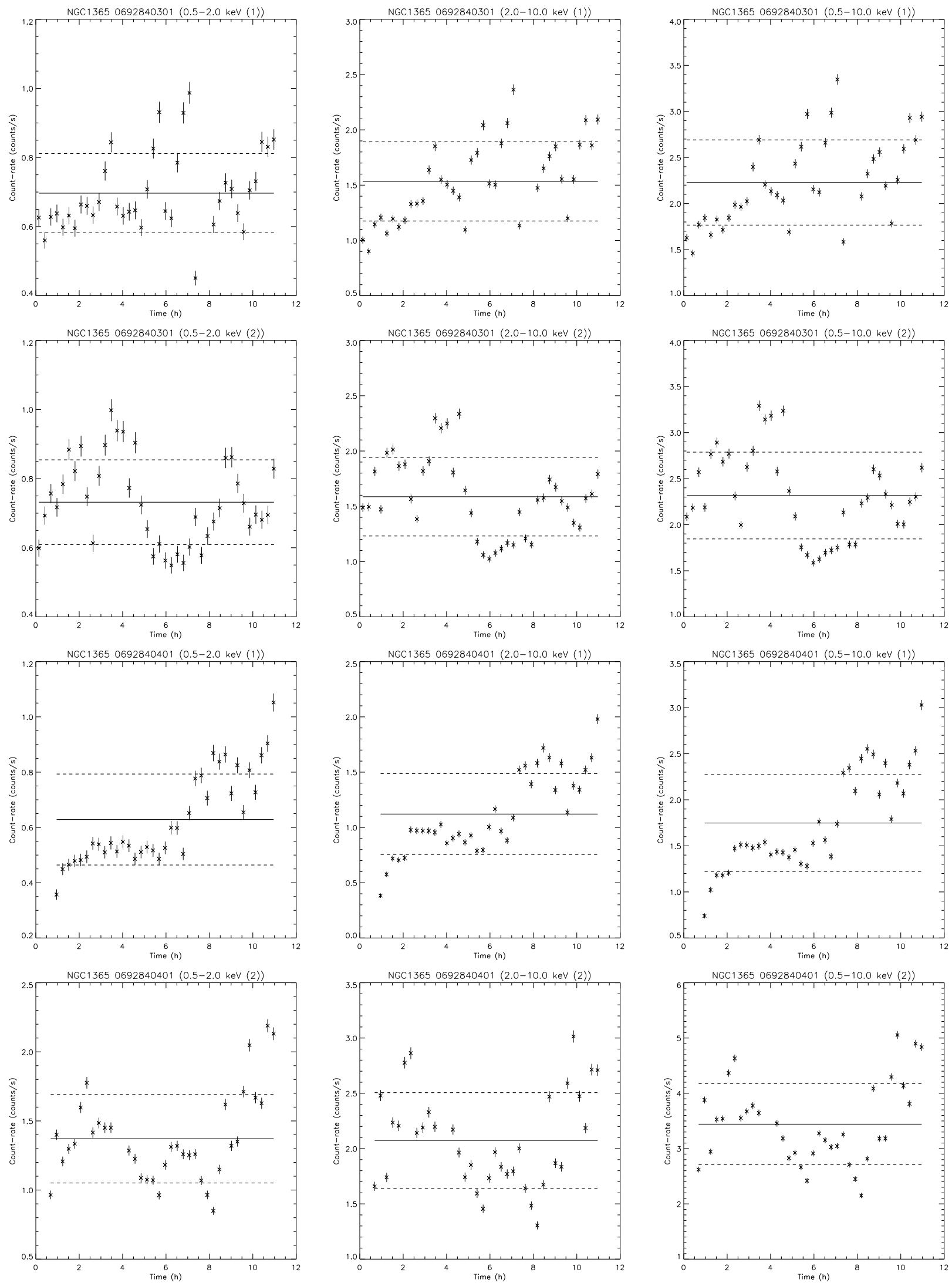

Fig. D.3. continued. 
A\&A 602, A65 (2017)
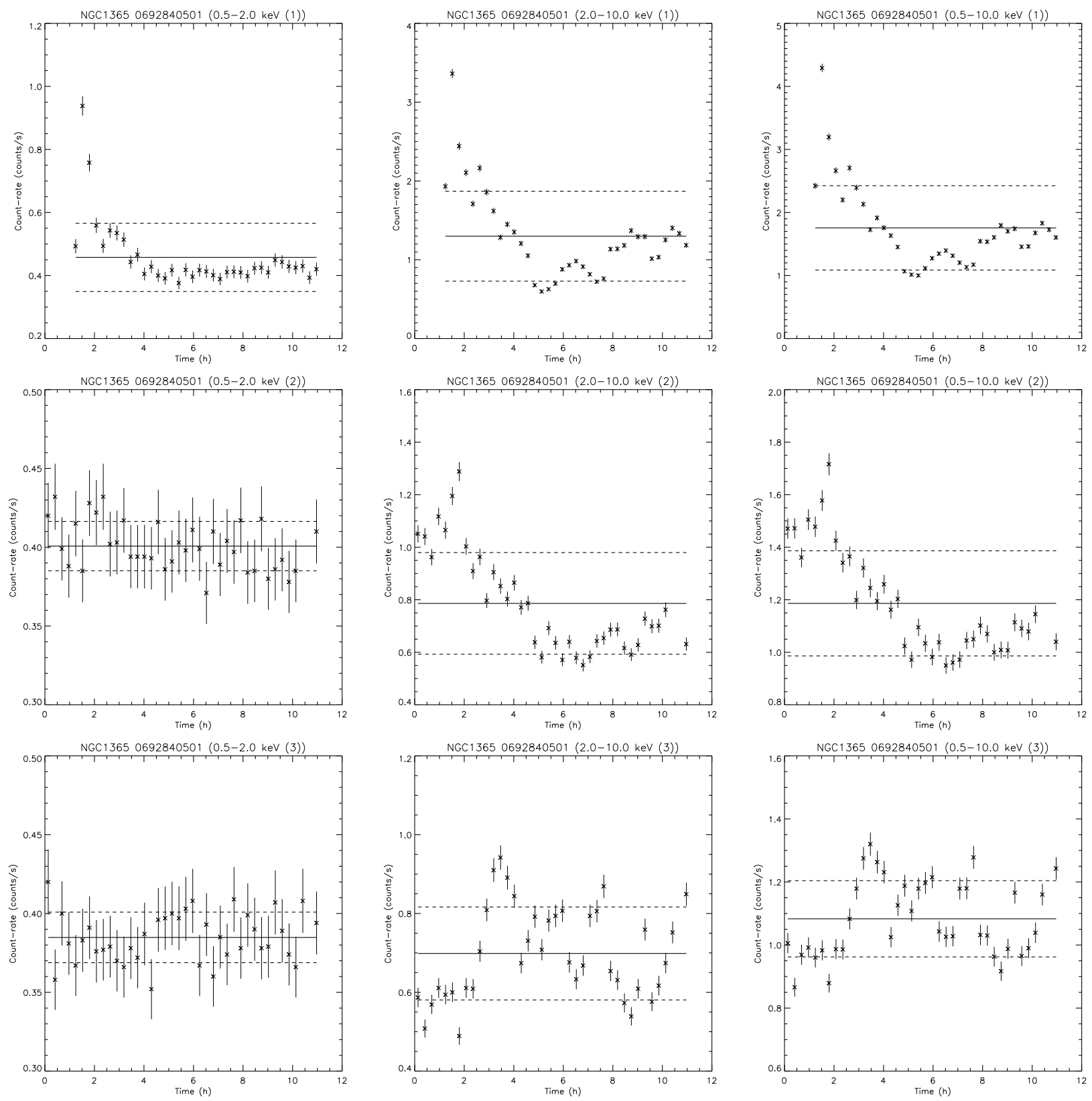

Fig. D.3. continued.
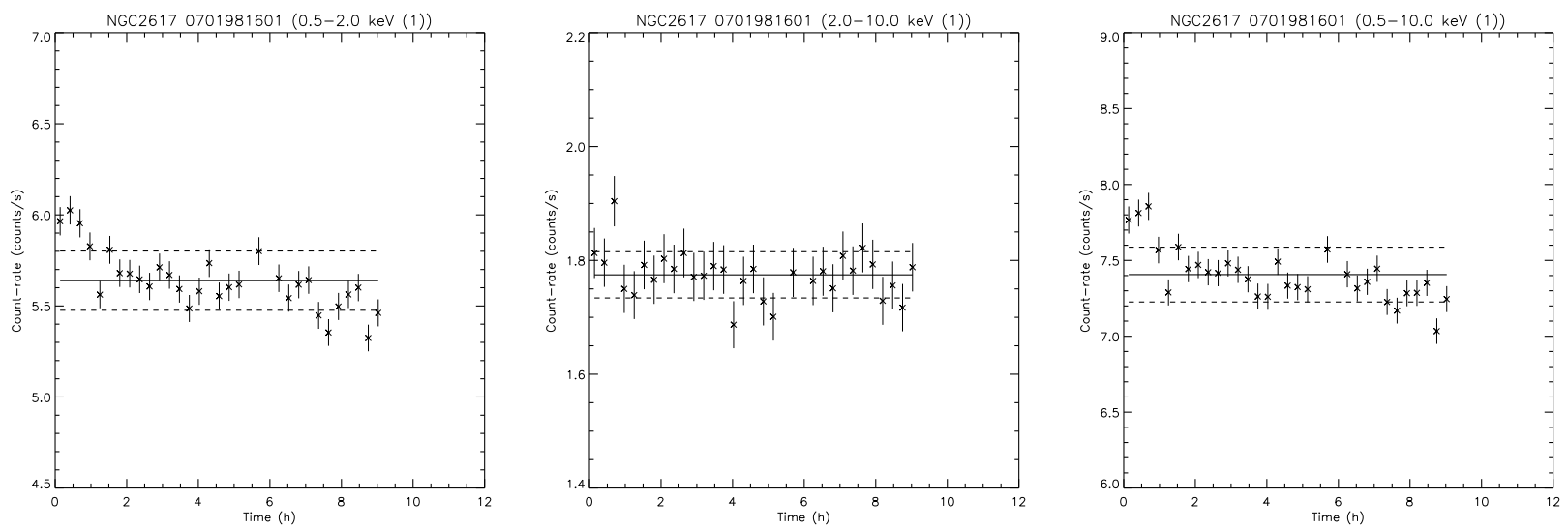

Fig. D.4. Light curves of NGC 2617 from XMM-Newton data. 
L. Hernández-García et al.: X-ray variability in Seyfert 1.8/1.9 galaxies
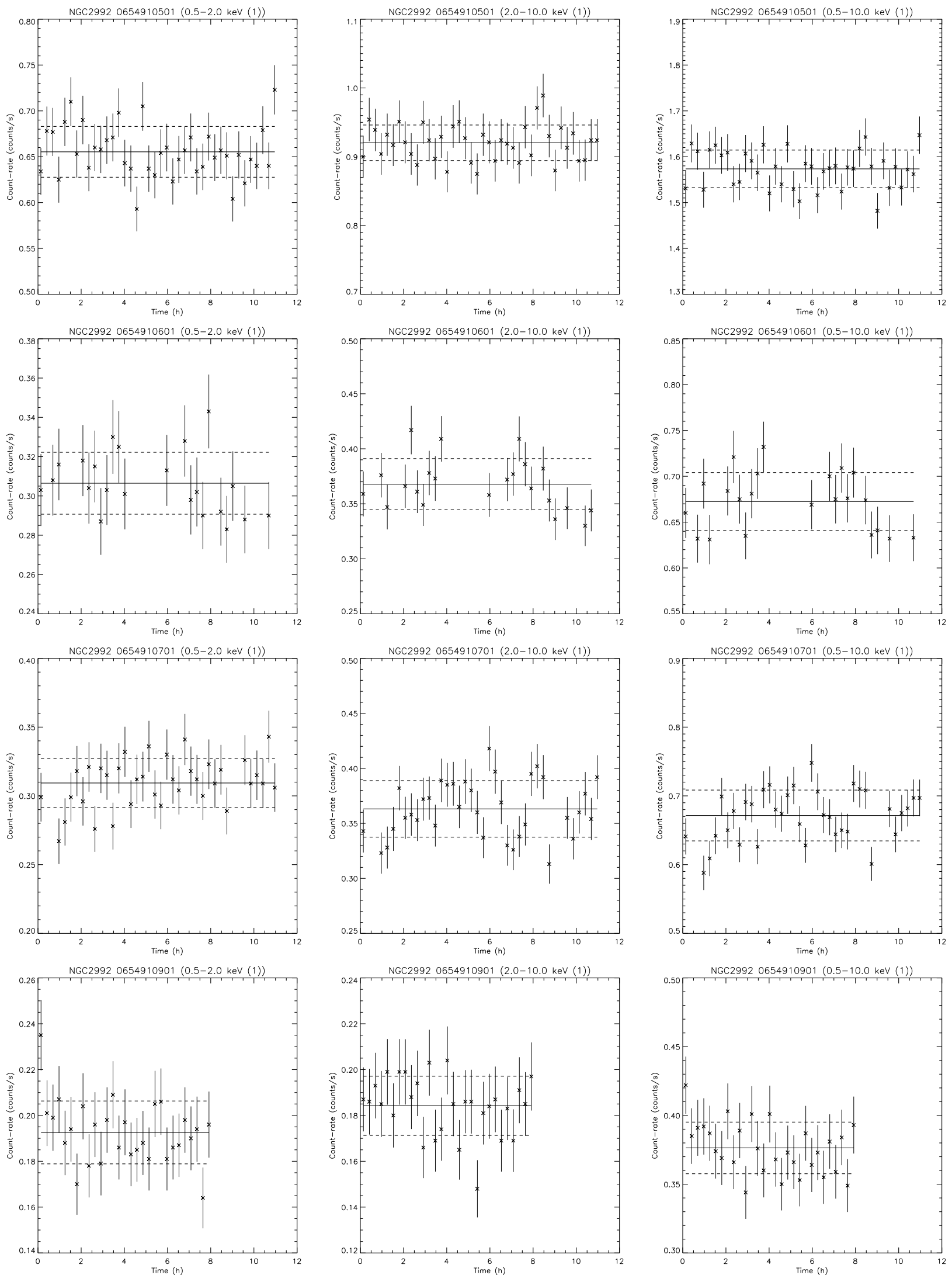

Fig. D.5. Light curves of NGC 2992 from XMM-Newton data. 
A\&A 602, A65 (2017)
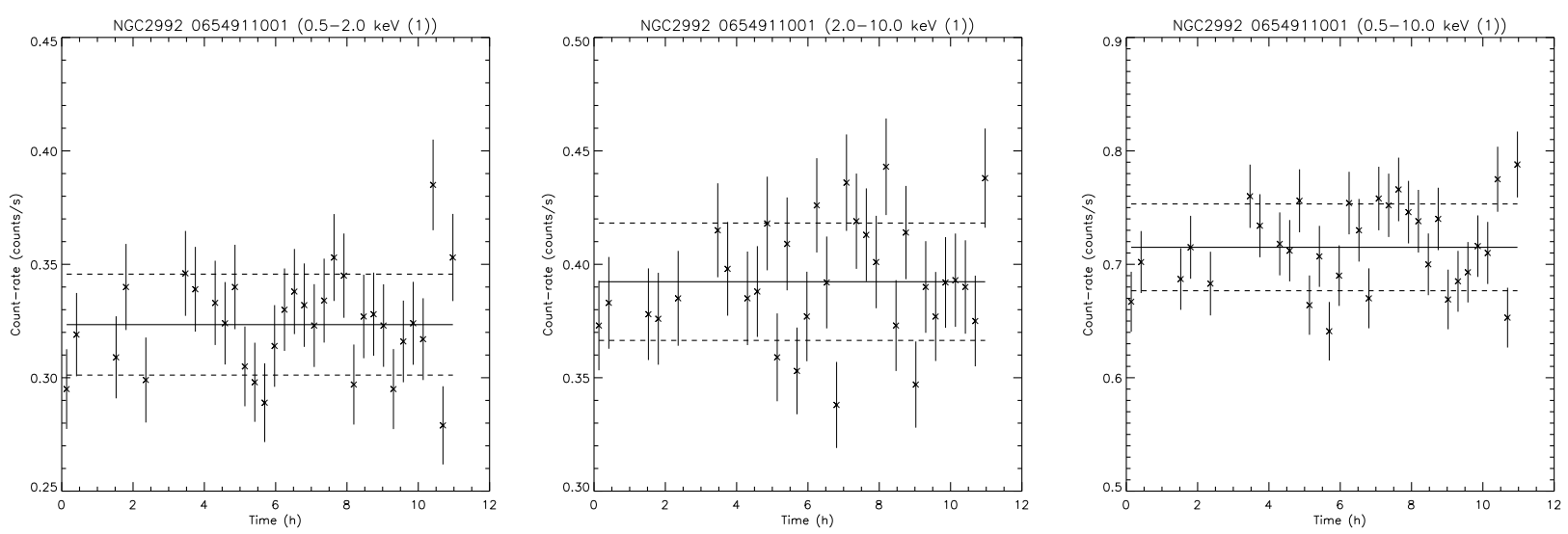

Fig. D.5. continued.
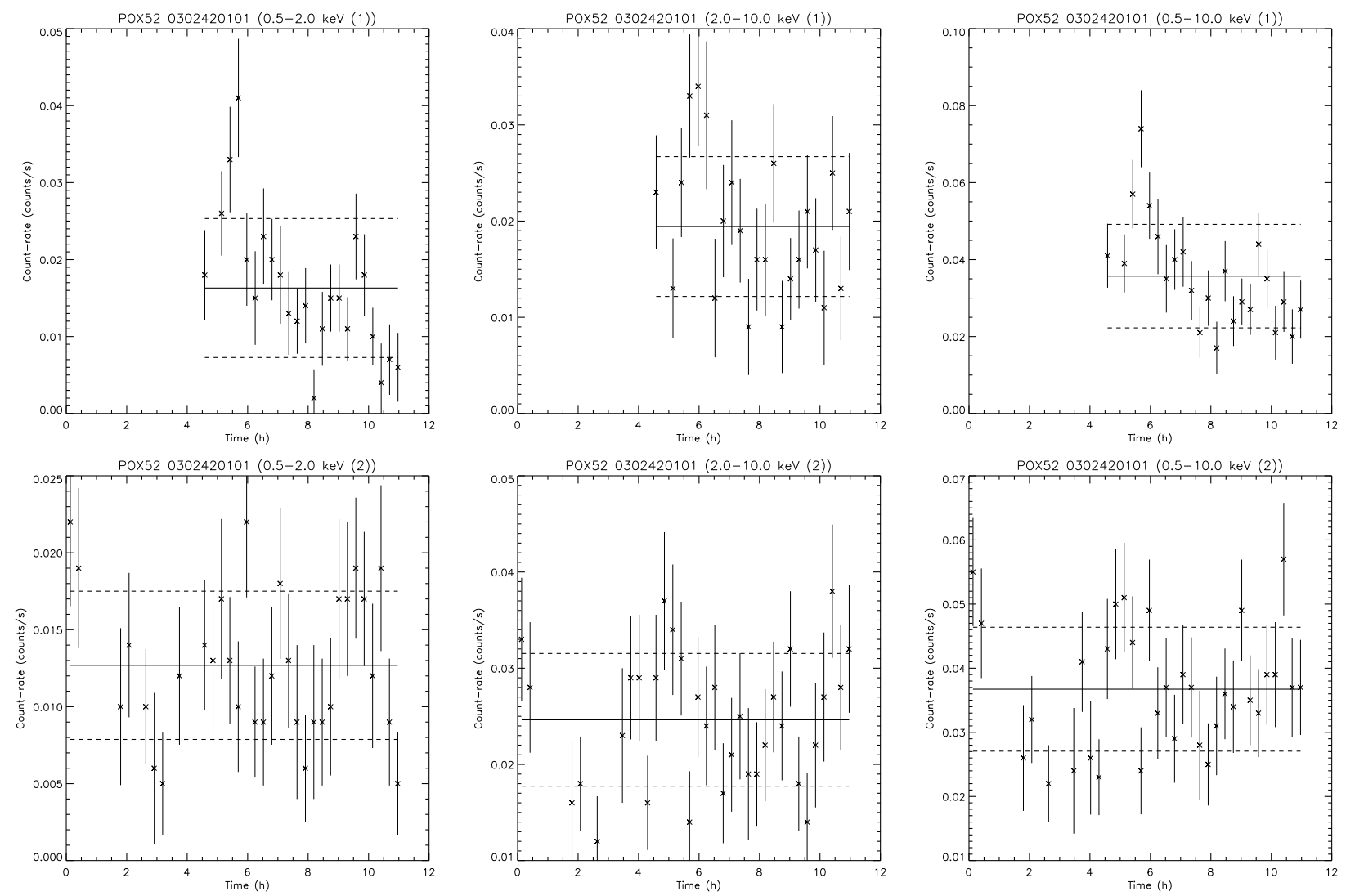

Fig. D.6. Light curves of POX 52 from XMM-Newton data.
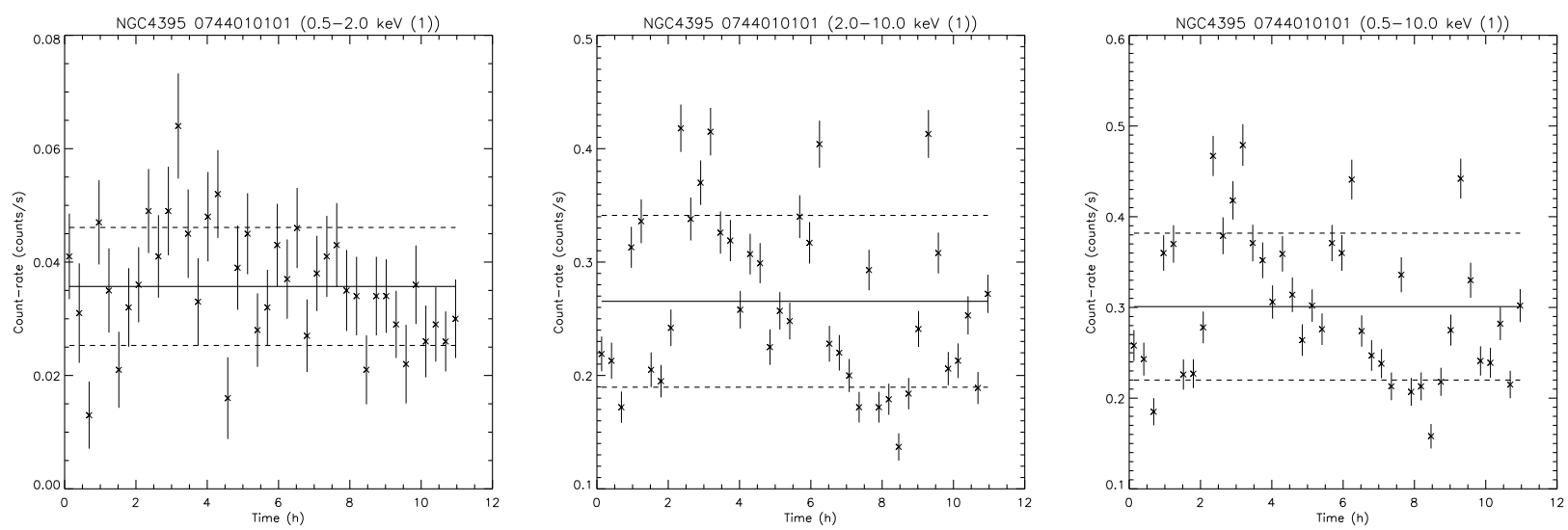

Fig. D.7. Light curves of NGC 4395 from XMM-Newton data. 
L. Hernández-García et al.: X-ray variability in Seyfert 1.8/1.9 galaxies
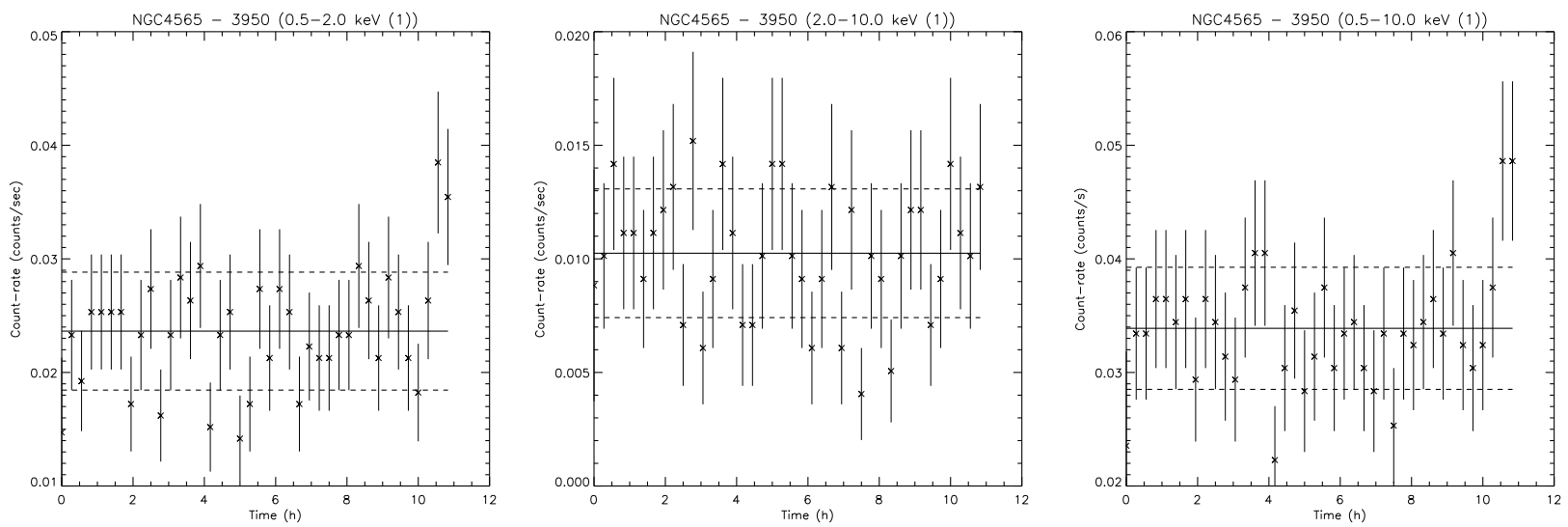

Fig. D.8. Light curves of NGC 4565 from Chandra data. 